\title{
On the Localized Phase of a Copolymer in an Emulsion: Supercritical Percolation Regime
}

\author{
F. den Hollander ${ }^{1,2}$, N. Pétrélis ${ }^{2}$ \\ ${ }^{1}$ Mathematical Institute, Leiden University, P.O. Box 9512, 2300 RA Leiden, The Netherlands \\ 2 EURANDOM, P.O. Box 513, 5600 MB Eindhoven, The Netherlands. \\ E-mail: petrelis@math.tu-berlin.de
}

Received: 15 October 2007 / Accepted: 18 July 2008

Published online: 19 November 2008 - (c) The Author(s) 2008. This article is published with open access at Springerlink.com

\begin{abstract}
In this paper we study a two-dimensional directed self-avoiding walk model of a random copolymer in a random emulsion. The copolymer is a random concatenation of monomers of two types, $A$ and $B$, each occurring with density $\frac{1}{2}$. The emulsion is a random mixture of liquids of two types, $A$ and $B$, organised in large square blocks occurring with density $p$ and $1-p$, respectively, where $p \in(0,1)$. The copolymer in the emulsion has an energy that is minus $\alpha$ times the number of $A A$-matches minus $\beta$ times the number of $B B$-matches, where without loss of generality the interaction parameters can be taken from the cone $\left\{(\alpha, \beta) \in \mathbb{R}^{2}: \alpha \geq|\beta|\right\}$. To make the model mathematically tractable, we assume that the copolymer is directed and can only enter and exit a pair of neighbouring blocks at diagonally opposite corners.

In [7], a variational expression was derived for the quenched free energy per monomer in the limit as the length $n$ of the copolymer tends to infinity and the blocks in the emulsion have size $L_{n}$ such that $L_{n} \rightarrow \infty$ and $L_{n} / n \rightarrow 0$. Under this restriction, the free energy is self-averaging with respect to both types of randomness. It was found that in the supercritical percolation regime $p \geq p_{c}$, with $p_{c}$ the critical probability for directed bond percolation on the square lattice, the free energy has a phase transition along a curve in the cone that is independent of $p$. At this critical curve, there is a transition from a phase where the copolymer is fully delocalized into the $A$-blocks to a phase where it is partially localized near the $A B$-interface. In the present paper we prove three theorems that complete the analysis of the phase diagram : (1) the critical curve is strictly increasing; (2) the phase transition is second order; (3) the free energy is infinitely differentiable throughout the partially localized phase.

In the subcritical percolation regime $p<p_{c}$, the phase diagram is much more complex. This regime will be treated in a forthcoming paper.
\end{abstract}

\section{Introduction and Main Results}

1.1. Background. The problem considered in this paper is the localization transition of a random copolymer near a random interface. Suppose that we have two immiscible 
liquids, say, oil and water, and a copolymer chain consisting of two types of monomer, say, hydrophobic and hydrophilic. Suppose that it is energetically favourable for monomers of one type to be in one liquid and for monomers of the other type to be in the other liquid. At high temperatures the copolymer will delocalize into one of the liquids in order to maximise its entropy, while at low temperatures energetic effects will dominate and the copolymer will localize close to the interface between the two liquids, because in this way it is able to place more than half of its monomers in their preferred liquid. In the limit as the copolymer becomes long, we may expect a phase transition.

In the literature most attention has focussed on models with a single flat infinite interface or an infinite array of parallel flat infinite interfaces. Relevant references can be found in the monograph by Giacomin [4] and in the theses by Caravenna [3] and Pétrélis [9]. In the present paper we continue the analysis of a model introduced in den Hollander and Whittington [7], where the interface has a random shape. In particular, the situation was considered in which the square lattice is divided into large blocks, and each block is independently labelled $A$ (oil) or $B$ (water) with probability $p$ and $1-p$, respectively, i.e., the interface has a percolation type structure. This is a primitive model of an emulsion, consisting of oil droplets dispersed in water (see Fig. 1).

The copolymer consists of an i.i.d. random concatenation of monomers of type $A$ (hydrophobic) and $B$ (hydrophilic). It is energetically favourable for monomers of type $A$ to be in the $A$-blocks and for monomers of type $B$ to be in the $B$-blocks. Under the restriction that the copolymer is directed and can only enter and exit a pair of neighbouring blocks at diagonally opposite corners, it was shown that there are phase transitions between phases where the copolymer is fully delocalized away from the interface and phases where it is partially localized near the interface. Let $p_{c} \approx 0.64$ be the critical probability for directed bond percolation on the square lattice. It turns out that the phase diagram does not depend on $p$ when $p \geq p_{c}$, while it does depend on $p$ when $p<p_{c}$. In the present paper we focus on the supercritical percolation regime, i.e., $p \geq p_{c}$.

Our paper is organised as follows. In the rest of Sect. 1 we recall the definition of the model, state the relevant results from [7], and formulate three theorems for the supercritical percolation regime. These theorems are proved in Sects. 3, 4 and 5, respectively. Section 2 recalls the key variational formula for the free energy, as well as some basic facts about block pair free energies and path entropies needed along the way.

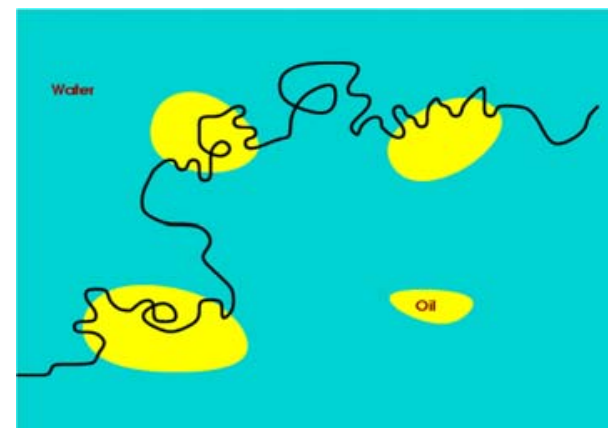

Fig. 1. An undirected copolymer in an emulsion 
1.2. The model. Each positive integer is randomly labelled $A$ or $B$, with probability $\frac{1}{2}$ each, independently for different integers. The resulting labelling is denoted by

$$
\omega=\left\{\omega_{i}: i \in \mathbb{N}\right\} \in\{A, B\}^{\mathbb{N}}
$$

and represents the randomness of the copolymer, with $A$ denoting a hydrophobic monomer and $B$ a hydrophilic monomer. Fix $p \in(0,1)$ and $L_{n} \in \mathbb{N}$. Partition $\mathbb{R}^{2}$ into square blocks of size $L_{n}$ :

$$
\mathbb{R}^{2}=\bigcup_{x \in \mathbb{Z}^{2}} \Lambda_{L_{n}}(x), \quad \Lambda_{L_{n}}(x)=x L_{n}+\left(0, L_{n}\right]^{2} .
$$

Each block is randomly labelled $A$ or $B$, with probability $p$, respectively, $1-p$, independently for different blocks. The resulting labelling is denoted by

$$
\Omega=\left\{\Omega(x): x \in \mathbb{Z}^{2}\right\} \in\{A, B\}^{\mathbb{Z}^{2}}
$$

and represents the randomness of the emulsion, with $A$ denoting oil and $B$ denoting water.

Let

- $\quad \mathcal{W}_{n}=$ the set of $n$-step directed self-avoiding paths starting at the origin and being allowed to move upwards, downwards and to the right.

- $\mathcal{W}_{n, L_{n}}=$ the subset of $\mathcal{W}_{n}$ consisting of those paths that enter blocks at a corner, exit blocks at one of the two corners diagonally opposite the one where it entered, and in between stay confined to the two blocks that are seen upon entering (see Fig. 2).

The corner restriction, which is unphysical, is put in to make the model mathematically tractable. We will see that, despite this restriction, the model has physically relevant behaviour.

Given $\omega, \Omega$ and $n$, with each path $\pi \in \mathcal{W}_{n, L_{n}}$ we associate an energy given by the Hamiltonian

$$
H_{n, L_{n}}^{\omega, \Omega}(\pi)=-\sum_{i=1}^{n}\left(\alpha 1\left\{\omega_{i}=\Omega_{\left(\pi_{i-1}, \pi_{i}\right)}^{L_{n}}=A\right\}+\beta 1\left\{\omega_{i}=\Omega_{\left(\pi_{i-1}, \pi_{i}\right)}^{L_{n}}=B\right\}\right),
$$

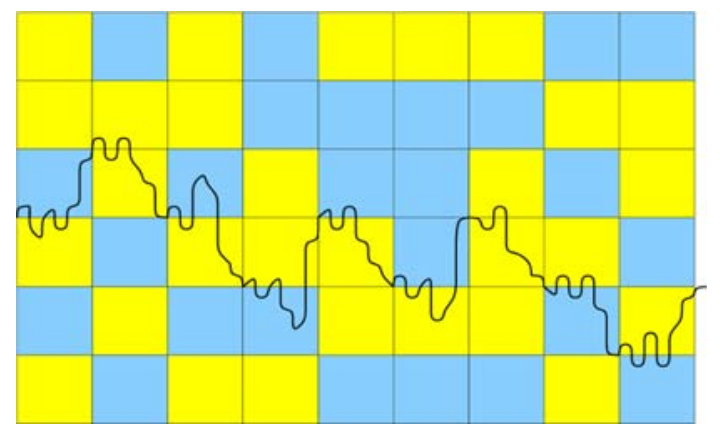

Fig. 2. A directed self-avoiding path crossing blocks of oil and water diagonally. The light-shaded blocks are oil, the dark-shaded blocks are water. Each block is $L_{n}$ lattice spacings wide in both directions. The path carries hydrophobic and hydrophilic monomers on the lattice scale, which are not indicated 
where $\left(\pi_{i-1}, \pi_{i}\right)$ denotes the $i_{t h}$ step of the path and $\Omega_{\left(\pi_{i-1}, \pi_{i}\right)}^{L_{n}}$ denotes the label of the block this step lies in. What this Hamiltonian does is count the number of $A A$ matches and $B B$-matches and assign them energy $-\alpha$ and $-\beta$, respectively, where $\alpha, \beta \in \mathbb{R}$. (Note that the interaction is assigned to bonds rather than to sites: we identify the monomers with the steps of the path). As we will recall in Sect. 2.1, without loss of generality we may restrict the interaction parameters to the cone

$$
\mathrm{CONE}=\left\{(\alpha, \beta) \in \mathbb{R}^{2}: \alpha \geq|\beta|\right\} .
$$

Given $\omega, \Omega$ and $n$, we define the quenched free energy per step as

$$
\begin{aligned}
f_{n, L_{n}}^{\omega, \Omega} & =\frac{1}{n} \log Z_{n, L_{n}}^{\omega, \Omega}, \\
Z_{n, L_{n}}^{\omega, \Omega} & =\sum_{\pi \in \mathcal{W}_{n, L_{n}}} \exp \left[-H_{n, L_{n}}^{\omega, \Omega}(\pi)\right] .
\end{aligned}
$$

We are interested in the limit $n \rightarrow \infty$ subject to the restriction

$$
L_{n} \rightarrow \infty \quad \text { and } \quad \frac{1}{n} L_{n} \rightarrow 0 .
$$

This is a coarse-graining limit where the path spends a long time in each single block yet visits many blocks. In this limit, there is a separation between a copolymer scale and an emulsion scale.

In [7], Theorem 1.3.1, it was shown that

$$
\lim _{n \rightarrow \infty} f_{n, L_{n}}^{\omega, \Omega}=f=f(\alpha, \beta ; p)
$$

exists $\omega, \Omega$-a.s. and in mean, is finite and non-random, and can be expressed as a variational problem involving the free energies of the copolymer in each of the four block pairs it may encounter and the frequencies at which the copolymer visits each of these block pairs on the coarse-grained block scale. This variational problem, which is recalled in Sect. 2.1, will be the starting point of our analysis.

1.3. Phase diagram for $p \geq p_{c}$. In the supercritical regime the oil blocks percolate, and so the coarse-grained path can choose between moving into the oil or running along the interface between the oil and the water (see Fig. 3). We begin by recalling from den Hollander and Whittington [7] the two main theorems for the supercritical percolation regime (see Fig. 4).

Theorem 1.3.1. ([7], Theorem 1.4.1). Let $p \geq p_{c}$. Then $(\alpha, \beta) \mapsto f(\alpha, \beta ; p)$ is nonanalytic along the curve in CONE separating the two regions

$$
\begin{aligned}
& \mathcal{D}=\text { delocalized phase }=\left\{(\alpha, \beta) \in \mathrm{CONE}: f(\alpha, \beta ; p)=\frac{1}{2} \alpha+\varpi\right\}, \\
& \mathcal{L}=\text { localized phase }=\left\{(\alpha, \beta) \in \mathrm{CONE}: f(\alpha, \beta ; p)>\frac{1}{2} \alpha+\varpi\right\} .
\end{aligned}
$$

Here, $\varpi=\lim _{n \rightarrow \infty} \frac{1}{n} \log \left|\mathcal{W}_{n, L_{n}}\right|=\frac{1}{2} \log 5$ is the entropy per step of the walk subject to (1.2.7). 


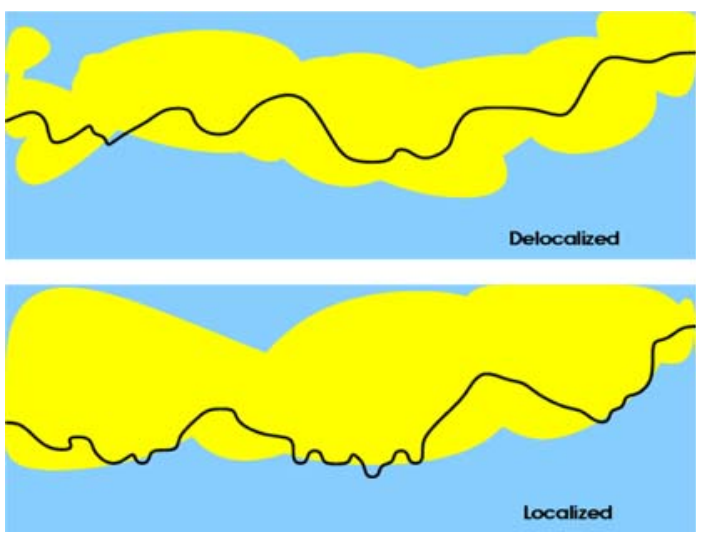

Fig. 3. Two possible strategies when the oil percolates

Theorem 1.3.2. ([7], Theorem 1.4.3). Let $p \geq p_{c}$.

(i) For every $\alpha \geq 0$ there exists a $\beta_{c}(\alpha) \in[0, \alpha]$ such that the copolymer is

$$
\begin{aligned}
& \text { delocalized if }-\alpha \leq \beta \leq \beta_{c}(\alpha), \\
& \text { localized if } \beta_{c}(\alpha)<\beta \leq \alpha .
\end{aligned}
$$

(ii) $\alpha \mapsto \beta_{c}(\alpha)$ is independent of $p$, continuous, non-decreasing and concave on $[0, \infty)$. There exist $\alpha^{*} \in(0, \infty)$ and $\beta^{*} \in\left[\alpha^{*}, \infty\right)$ such that

$$
\begin{aligned}
& \beta_{c}(\alpha)=\alpha \text { if } \alpha \leq \alpha^{*}, \\
& \beta_{c}(\alpha)<\alpha \text { if } \alpha>\alpha^{*},
\end{aligned}
$$

and

$$
\lim _{\alpha \downarrow \alpha^{*}} \frac{\alpha-\beta_{c}(\alpha)}{\alpha-\alpha^{*}} \in[0,1), \quad \lim _{\alpha \rightarrow \infty} \beta_{c}(\alpha)=\beta^{*} .
$$

The intuition behind Theorem 1.3.1 is as follows (see Fig. 3). Suppose that $p>p_{c}$. Then the $A$-blocks percolate. Therefore the copolymer has the option of moving to the infinite cluster of $A$-blocks and staying inside that infinite cluster forever, thus seeing only $A A$-blocks. In doing so, it loses an entropy of at most $O\left(n / L_{n}\right)=o(n)$ (on the coarse-grained scale), it gains an energy $\frac{1}{2} \alpha n+o(n)$ (on the lattice scale, because only half of its monomers are matched), and it gains an entropy $\varpi n+o(n)$ (on the lattice scale, because it crosses blocks diagonally). Alternatively, the path has the option of running along the boundary of the infinite cluster (at least part of the time), during which it sees $A B$-blocks and (when $\beta \geq 0$ ) gains more energy by matching more than half of its monomers. Consequently,

$$
f(\alpha, \beta ; p) \geq \frac{1}{2} \alpha+\varpi .
$$

The boundary between the two regimes in (1.3.1) corresponds to the crossover from full delocalization into the $A$-blocks to partial localization near the $A B$-interfaces. The critical curve does not depend on $p$ as long as $p>p_{c}$. Because $p \mapsto f(\alpha, \beta ; p)$ is continuous (see Theorem 2.1.1(iii) in Sect. 2.1), the same critical curve occurs at $p=p_{c}$. 
The proof of Theorem 1.3.2 relies on a representation of $\mathcal{D}$ and $\mathcal{L}$ in terms of the single interface (!) free energy (see Proposition 2.3.4 in Sect. 2.3). This representation, which is key to the analysis of the critical curve, expresses the fact that localization occurs for the emulsion free energy only when the single interface free energy is sufficiently deep inside its localized phase. This gap is needed to compensate for the loss of entropy associated with running along the interface and crossing at a steeper angle.

The intuition behind Theorem 1.3.2 is as follows (see Fig. 4). Pick a point $(\alpha, \beta)$ inside $\mathcal{D}$. Then the copolymer spends almost all of its time deep inside the $A$-blocks. Increase $\beta$ while keeping $\alpha$ fixed. Then there will be a larger energetic advantage for the copolymer to move some of its monomers from the $A$-blocks to the $B$-blocks by crossing the interface inside the $A B$-block pairs. There is some entropy loss associated with doing so, but if $\beta$ is large enough, then the energetic advantage will dominate, so that $A B$ localization sets in. The value at which this happens depends on $\alpha$ and is strictly positive. Since the entropy loss is finite, for $\alpha$ large enough the energy-entropy competition plays out not only below the diagonal, but also below a horizontal asymptote. On the other hand, for $\alpha$ small enough the loss of entropy dominates the energetic advantage, which is why the critical curve has a piece that lies on the diagonal. The larger the value of $\alpha$ the larger the value of $\beta$ where $A B$-localization sets in. This explains why the critical curve is non-decreasing. At the critical curve the single interface free energy is already inside its localized phase. This explains why the critical curve has a slope discontinuity at $\alpha^{*}$.

1.4. Main results. In the present paper we prove three theorems, which complete the analysis of the phase diagram in Fig. 4.

Theorem 1.4.1. Let $p \geq p_{c}$. Then $\alpha \mapsto \beta_{c}(\alpha)$ is strictly increasing on $[0, \infty)$.

Theorem 1.4.2. Let $p \geq p_{c}$. Then for every $\alpha \in\left(\alpha^{*}, \infty\right)$ there exist $0<C_{1}<C_{2}<\infty$ and $\delta_{0}>0$ (depending on $p$ and $\alpha$ ) such that

$$
C_{1} \delta^{2} \leq f\left(\alpha, \beta_{c}(\alpha)+\delta ; p\right)-f\left(\alpha, \beta_{c}(\alpha) ; p\right) \leq C_{2} \delta^{2} \quad \forall \delta \in\left(0, \delta_{0}\right] .
$$

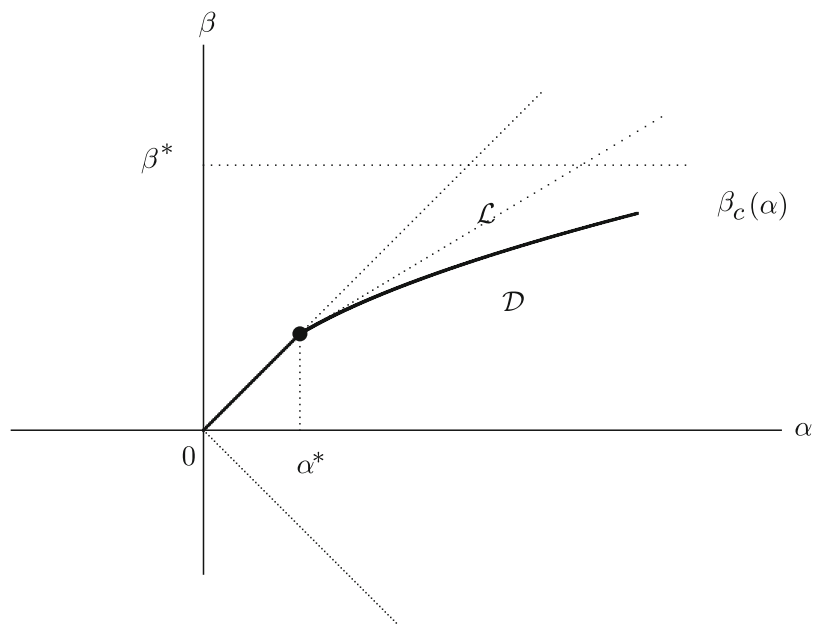

Fig. 4. Qualitative picture of $\alpha \mapsto \beta_{c}(\alpha)$ for $p \geq p_{c}$ 
Theorem 1.4.3. Let $p \geq p_{c}$. Then, under Assumption 5.2.2, $(\alpha, \beta) \mapsto f(\alpha, \beta ; p)$ is infinitely differentiable throughout $\mathcal{L}$.

Assumption 5.2.2 states that a certain intermediate single-interface free energy has a finite curvature. We believe this assumption to be true, but have not managed to prove it. See the end of Sect. 5.2, in particular, Remark 5.3.3, for a motivation and for a way to weaken it.

Theorem 1.4.1 implies that the critical curve never reaches the horizontal asymptote, which in turn implies that $\alpha^{*}<\beta^{*}$ and that the slope in (1.3.4) is $>0$. Theorem 1.4.2 shows that the phase transition is second order off the diagonal. (In contrast, we know that the phase transition is first order on the diagonal. Indeed, the free energy equals $\frac{1}{2} \alpha+\varpi$ on and below the diagonal segment between $(0,0)$ and $\left(\alpha^{*}, \alpha^{*}\right)$, and equals $\frac{1}{2} \beta+\varpi$ on and above this segment as is evident from interchanging $\alpha$ and $\beta$.) Theorem 1.4.3 tells us that the critical curve is the only location in CONE where a phase transition of finite order occurs. Theorems 1.4.1, 1.4.2 and 1.4.3 are proved in Sects. 3, 4 and 5, respectively. Their proofs rely on perturbation arguments, in combination with exponential tightness of the excursions away from the interface inside the localized phase.

The analogues of Theorems 1.4.2 and 1.4.3 for the single flat infinite interface were derived in Giacomin and Toninelli [5,6]. For that model the phase transition is shown to be at least of second order, i.e., only the quadratic upper bound is proved. Numerical simulation indicates that the transition may well be of higher order.

The mechanisms behind the phase transition in the two models are different. While for the single interface model the copolymer makes long excursions away from the interface and dips below the interface during a fraction of time that is at most of order $\delta^{2}$, in our emulsion model the copolymer runs along the interface during a fraction of time that is of order $\delta$, and in doing so stays close to the interface. Morover, because near the critical curve for the emulsion model the single interface model is already inside its localized phase, there is a variation of order $\delta$ in the single interface free energy. Thus, the $\delta^{2}$ in the emulsion model is the product of two factors $\delta$, one coming from the time spent running along the interface and one coming from the variation of the constituent single interface free energy away from its critical curve. See Sect. 4 for more details.

In the proof of Theorem 1.4.3 we use some of the ingredients of the proof in Giacomin and Toninelli [6] of the analogous result for the single interface model. However, in the emulsion model there is an extra complication, namely, the speed per step to move one unit of space forward may vary (because steps are up, down and to the right), while in the single interface model this is fixed at one (because steps are up-right and down-right). We need to control the infinite differentiability with respect to this speed variable. This is done by considering the Fenchel-Legendre transform of the free energy, in which the dual of the speed variable enters into the Hamiltonian rather than in the set of paths. Moreover, since the block pair free energies and the total free energy are both given by variational problems, we need to show uniqueness of maximisers and prove non-degeneracy of the Jacobian matrix at these maximisers in order to be able to apply implicit function theorems. See Sect. 5 for more details.

1.5. Discussion. The corner restriction imposed through the set $\mathcal{W}_{n, L_{n}}$ in Sect. 1.2 is unphysical. However, without this restriction the model would be very hard to analyze, and would have a degree of difficulty comparable to that of the directed polymer in random environment, for which no detailed phase diagram has yet been derived. If the copolymer is allowed to exit a pair of blocks also at the corner to the right of the 
entrance corner, then this adds an extra critical curve to the phase diagram, namely, the critical curve of the single linear interface. Our critical curve still persists, because the copolymer has to cross $A B$-blocks diagonally every now and then in order to reach the most favorable block environment. The order of the phase transition at our critical curve is unaffected. The order of the extra critical curve would be the same as for the single linear interface, i.e., second order or higher.

\section{Preparations}

In Sects. 2.1-2.3 we recall a few key facts from den Hollander and Whittington [7] that will be crucial for the proofs. Section 2.1 gives the variational formula for the free energy, Sect. 2.2 states two elementary lemmas about path entropies, while Sect. 2.3 states two lemmas for the block pair free energies and a proposition characterising the localized phase of the emulsion free energy in terms of the single interface free energy. Section 2.4 states a lemma about the tail behaviour of the single interface free energy and the block pair free energies, showing that long paths wash out the effect of entropy.

2.1. Variational formula for the free energy. To formulate the key variational formula for the free energy that serves as our starting point, we need three ingredients.

I. For $L \in \mathbb{N}$ and $a \geq 2$ (with $a L$ integer), let $\mathcal{W}_{a L, L}$ denote the set of $a L$-step directed self-avoiding paths starting at $(0,0)$, ending at $(L, L)$, and in between not leaving the two adjacent blocks of size $L$ labelled $(0,0)$ and $(-1,0)$ (see Fig. 5). For $k, l \in\{A, B\}$, let

$$
\begin{aligned}
\psi_{k l}^{\omega}(a L, L) & =\frac{1}{a L} \log Z_{a L, L}^{\omega}, \\
Z_{a L, L}^{\omega} & =\sum_{\pi \in \mathcal{W}_{a L, L}} \exp \left[-H_{a L, L}^{\omega, \Omega}(\pi)\right] \text { when } \Omega(0,0)=k \text { and } \Omega(0,-1)=l,
\end{aligned}
$$

denote the free energy per step in a $k l$-block when the number of steps inside the block is $a$ times the size of the block. Let

$$
\lim _{L \rightarrow \infty} \psi_{k l}^{\omega}(a L, L)=\psi_{k l}(a)=\psi_{k l}(\alpha, \beta ; a)
$$

Note here that $k$ labels the type of the block that is diagonally crossed, while $l$ labels the type of the block that appears as its neighbour at the starting corner (see Fig. 5). We will recall in Sect. 2.3 that the limit exists $\omega$-a.s. and in mean, and is non-random. Both $\psi_{A A}$ and $\psi_{B B}$ take on a simple form, whereas $\psi_{A B}$ and $\psi_{B A}$ do not.

II. Let $\mathcal{W}$ denote the class of all coarse-grained paths $\Pi=\left\{\Pi_{j}: j \in \mathbb{N}\right\}$ that step diagonally from corner to corner (see Fig. 4, where each dashed line with arrow denotes a single step of $\Pi)$. For $n \in \mathbb{N}, \Pi \in \mathcal{W}$ and $k, l \in\{A, B\}$, let

$$
\begin{aligned}
& \rho_{k l}^{\Omega}(\Pi, n) \\
& =\frac{1}{n} \sum_{j=1}^{n} 1\left\{\begin{array}{l}
\left(\Pi_{j-1}, \Pi_{j}\right) \text { diagonally crosses a } k \text {-block in } \Omega \text { that has an } l \text {-block } \\
\text { in } \Omega \text { appearing as its neighbour at the starting corner }
\end{array}\right\} .
\end{aligned}
$$




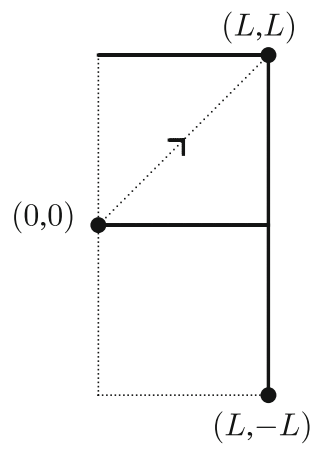

Fig. 5. Two neighbouring blocks. The dashed line with arrow indicates that the coarse-grained path makes a step diagonally upwards. The path enters at $(0,0)$, exits at $(L, L)$, and in between stays confined to the two blocks

Abbreviate

$$
\rho^{\Omega}(\Pi, n)=\left(\rho_{k l}^{\Omega}(\Pi, n)\right)_{k, l \in\{A, B\}},
$$

which is a $2 \times 2$ matrix with non-negative elements that sum up to 1 . Let $\mathcal{R}^{\Omega}(\Pi)$ denote the set of all limits points of the sequence $\left\{\rho^{\Omega}(\Pi, n): n \in \mathbb{N}\right\}$, and put

$$
\mathcal{R}^{\Omega}=\text { the closure of the set } \bigcup_{\Pi \in \mathcal{W}} \mathcal{R}^{\Omega}(\Pi) .
$$

Clearly, $\mathcal{R}^{\Omega}$ exists for all $\Omega$. Moreover, since $\Omega$ has a trivial sigma-field at infinity (i.e., all events not depending on finitely many coordinates of $\Omega$ have probability 0 or 1 ) and $\mathcal{R}^{\Omega}$ is measurable with respect to this sigma-field, we have

$$
\mathcal{R}^{\Omega}=\mathcal{R}(p) \quad \Omega-a . s .
$$

for some non-random closed set $\mathcal{R}(p)$. This set, which depends on the parameter $p$ controlling $\Omega$, is the set of all possible limit points of the frequencies at which the four pairs of adjacent blocks can be seen along an infinite coarse-grained path. The elements of $\mathcal{R}(p)$ are matrices

$$
\left(\begin{array}{cc}
\rho_{A A} & \rho_{A B} \\
\rho_{B A} & \rho_{B B}
\end{array}\right)
$$

whose elements are non-negative and sum up to 1. In [7], Proposition 3.2.1, it was shown that $p \mapsto \mathcal{R}(p)$ is continuous in the Hausdorff metric and that, for $p \geq p_{c}, \mathcal{R}(p)$ contains matrices of the form

$$
M_{\gamma}=\left(\begin{array}{lr}
1-\gamma & \gamma \\
0 & 0
\end{array}\right) \quad \text { for } \gamma \in C \subset(0,1) \text { closed. }
$$

III. Let $\mathcal{A}$ be the set of $2 \times 2$ matrices whose elements are $\geq 2$. The elements of these matrices are used to record the average number of steps made by the path inside the four block pairs divided by the block size.

With I-III in hand, we can state the variational formula for the free energy. Define

$$
V:\left(\left(\rho_{k l}\right),\left(a_{k l}\right)\right) \in \mathcal{R}(p) \times \mathcal{A} \mapsto \frac{\sum_{k l} \rho_{k l} a_{k l} \psi_{k l}\left(a_{k l}\right)}{\sum_{k l} \rho_{k l} a_{k l}} .
$$


Theorem 2.1.1. ([7], Theorem 1.3.1).

(i) For all $(\alpha, \beta) \in \mathbb{R}^{2}$ and $p \in(0,1)$,

$$
\lim _{n \rightarrow \infty} f_{n, L_{n}}^{\omega, \Omega}=f=f(\alpha, \beta ; p)
$$

exists $\omega, \Omega$-a.s. and in mean, is finite and non-random, and is given by

$$
f=\sup _{\left(\rho_{k l}\right) \in \mathcal{R}(p)} \sup _{\left(a_{k l}\right) \in \mathcal{A}} V\left(\left(\rho_{k l}\right),\left(a_{k l}\right)\right) .
$$

(ii) $(\alpha, \beta) \mapsto f(\alpha, \beta ; p)$ is convex on $\mathbb{R}^{2}$ for all $p \in(0,1)$.

(iii) $p \mapsto f(\alpha, \beta ; p)$ is continuous on $(0,1)$ for all $(\alpha, \beta) \in \mathbb{R}^{2}$.

(iv) For all $(\alpha, \beta) \in \mathbb{R}^{2}$ and $p \in(0,1)$,

$$
\begin{aligned}
& f(\alpha, \beta ; p)=f(\beta, \alpha ; 1-p), \\
& f(\alpha, \beta ; p)=\frac{1}{2}(\alpha+\beta)+f(-\beta,-\alpha ; p) .
\end{aligned}
$$

Part (iv) is the reason why without loss of generality we may restrict the parameters to the cone in (1.2.5).

The behaviour of $f$ as a function of $(\alpha, \beta)$ is different for $p \geq p_{c}$ and $p<p_{c}$ (recall that $p_{c}$ is the critical probability for directed bond percolation on the square lattice). The reason is that the coarse-grained paths $\Pi$, which determine the set $\mathcal{R}(p)$, sample $\Omega$ just like paths in directed bond percolation on the square lattice rotated by 45 degrees sample the percolation configuration (see Fig. 6).

2.2. Path entropies. The two lemmas in this section identify the path entropies associated with crossing a block and running along an interface. They are based on straightforward computations and are crucial for the analysis of the model.

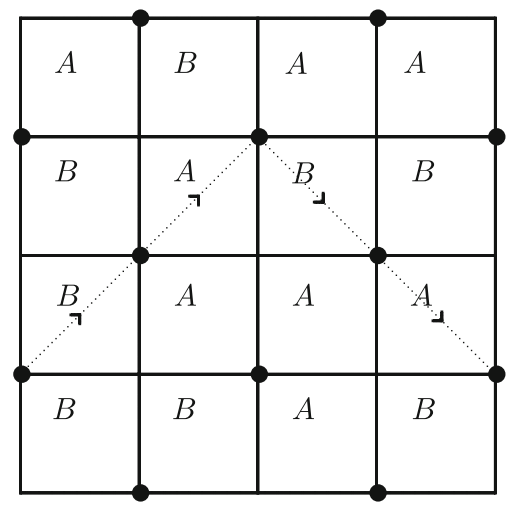

Fig. 6. $\Pi$ sampling $\Omega$. The dashed lines with arrows indicate the steps of $\Pi$. The block pairs encountered in this example are $B B, A A, B A$ and $A B$ 
Let

$$
\mathrm{DOM}=\{(a, b): a \geq 1+b, b \geq 0\} .
$$

For $(a, b) \in \mathrm{DOM}$, let $N_{L}(a, b)$ denote the number of $a L$-step self-avoiding directed paths from $(0,0)$ to $(b L, L)$ whose vertical displacement stays within $(-L, L](a L$ and $b L$ are integer). Let

$$
\kappa(a, b)=\lim _{L \rightarrow \infty} \frac{1}{a L} \log N_{L}(a, b)
$$

Lemma 2.2.1. ([7], Lemma 2.1.1).

(i) $\kappa(a, b)$ exists and is finite for all $(a, b) \in$ DOM.

(ii) $(a, b) \mapsto a \kappa(a, b)$ is continuous and strictly concave on DOM and analytic on the interior of DOM.

(iii) For all $a \geq 2$,

$$
a \kappa(a, 1)=\log 2+\frac{1}{2}[a \log a-(a-2) \log (a-2)] .
$$

(iv) $\sup _{a \geq 2} \kappa(a, 1)=\kappa\left(a^{*}, 1\right)=\frac{1}{2} \log 5$ with unique maximiser $a^{*}=\frac{5}{2}$.

(v) $\left(\frac{\partial}{\partial a} \kappa\right)\left(a^{*}, 1\right)=0$ and $a^{*}\left(\frac{\partial}{\partial b} \kappa\right)\left(a^{*}, 1\right)=\frac{1}{2} \log \frac{9}{5}$.

(vi) $\left(\frac{\partial^{2}}{\partial a^{2}} \kappa\right)\left(a^{*}, 1\right)=-\frac{8}{25},\left(\frac{\partial^{2}}{\partial b^{2}} \kappa\right)\left(a^{*}, 1\right)=-\frac{262}{225}$ and $\left(\frac{\partial^{2}}{\partial a \partial b} \kappa\right)\left(a^{*}, 1\right)=-\frac{2}{25} \log \frac{9}{5}+$ $\frac{44}{75}$.

Part (vi), which was not stated in [7], follows from a direct computation via [7], Eqs. (2.1.5), (2.1.8) and (2.1.9).

For $\mu \geq 1$, let $\hat{N}_{L}(\mu)$ denote the number of $\mu L$-step self-avoiding paths from $(0,0)$ to $(L, 0)$ with no restriction on the vertical displacement ( $\mu L$ is integer). Let

$$
\hat{\kappa}(\mu)=\lim _{L \rightarrow \infty} \frac{1}{\mu L} \log \hat{N}_{L}(\mu)
$$

Lemma 2.2.2. ([7], Lemma 2.1.2).

(i) $\hat{\kappa}(\mu)$ exists and is finite for all $\mu \geq 1$.

(ii) $\mu \mapsto \mu \hat{\kappa}(\mu)$ is continuous and strictly concave on $[1, \infty)$ and analytic on $(1, \infty)$.

(iii) $\hat{\kappa}(1)=0$ and $\mu \hat{\kappa}(\mu) \sim \log \mu$ as $\mu \rightarrow \infty$.

(iv) $\sup _{\mu \geq 1} \mu\left[\hat{\kappa}(\mu)-\frac{1}{2} \log 5\right]<\frac{1}{2} \log \frac{9}{5}$.

2.3. Free energies per pair of blocks. In this section we identify the block pair free energies. In [7], Proposition 2.2.1, we showed that $\omega$-a.s. and in mean,

$$
\psi_{A A}(a)=\frac{1}{2} \alpha+\kappa(a, 1) \quad \text { and } \quad \psi_{B B}(a)=\frac{1}{2} \beta+\kappa(a, 1) .
$$

Both are easy expressions, because $A A$-blocks and $B B$-blocks have no interface.

To compute $\psi_{A B}(a)$ and $\psi_{B A}(a)$, we first consider the free energy per step when the path moves in the vicinity of a single linear interface $\mathcal{I}$ separating a liquid $A$ in the upper halfplane from a liquid $B$ in the lower halfplane including the interface itself. To 
that end, for $c \geq b>0$, let $\mathcal{W}_{c L, b L}$ denote the set of $c L$-step directed self-avoiding paths starting at $(0,0)$ and ending at $(b L, 0)$. Define

$$
\psi_{L}^{\omega, \mathcal{I}}(c, b)=\frac{1}{c L} \log Z_{c L, b L}^{\omega, \mathcal{I}}
$$

with

$$
\begin{aligned}
Z_{c L, b L}^{\omega, \mathcal{I}} & =\sum_{\pi \in \mathcal{W}_{c L, b L}} \exp \left[-H_{c L}^{\omega, \mathcal{I}}(\pi)\right], \\
H_{c L}^{\omega, \mathcal{I}}(\pi) & =-\sum_{i=1}^{c L}\left(\alpha 1\left\{\omega_{i}=A,\left(\pi_{i-1}, \pi_{i}\right)>0\right\}+\beta 1\left\{\omega_{i}=B,\left(\pi_{i-1}, \pi_{i}\right) \leq 0\right\}\right),
\end{aligned}
$$

where $\left(\pi_{i-1}, \pi_{i}\right)>0$ means that the $i_{t h}$ step lies in the upper halfplane and $\left(\pi_{i-1}, \pi_{i}\right) \leq 0$ means that the $i_{t h}$ step lies in the lower halfplane or in the interface (see Fig. 7).

For $a \in[2, \infty)$, let

$$
\operatorname{DOM}(a)=\left\{(c, b) \in \mathbb{R}^{2}: 0 \leq b \leq 1, c \geq b, a-c \geq 2-b\right\} .
$$

Lemma 2.3.1. ([7], Lemma 2.2.1). For all $(\alpha, \beta) \in \mathbb{R}^{2}$ and $c \geq b>0$,

$$
\lim _{L \rightarrow \infty} \psi_{L}^{\omega, \mathcal{I}}(c, b)=\phi^{\mathcal{I}}(c / b)=\phi^{\mathcal{I}}(\alpha, \beta ; c / b)
$$

exists $\omega$-a.s. and in mean, and is non-random.

Lemma 2.3.2. ([7], Lemma 2.2.2). For all $(\alpha, \beta) \in \mathbb{R}^{2}$ and $a \geq 2$,

$$
\begin{aligned}
a \psi_{A B}(a) & =a \psi_{A B}(\alpha, \beta ; a) \\
& =\sup _{(c, b) \in \operatorname{DOM}(a)}\left\{c \phi^{\mathcal{I}}(c / b)+(a-c)\left[\frac{1}{2} \alpha+\kappa(a-c, 1-b)\right]\right\} .
\end{aligned}
$$

Lemma 2.3.3. ([7], Lemma 2.2.3). Let $k, l \in\{A, B\}$.

(i) For all $(\alpha, \beta) \in \mathbb{R}^{2}, a \mapsto a \psi_{k l}(\alpha, \beta ; a)$ is continuous and concave on $[2, \infty)$.

(ii) For all $a \in[2, \infty), \alpha \mapsto \psi_{k l}(\alpha, \beta ; a)$ and $\beta \mapsto \psi_{k l}(\alpha, \beta ; a)$ are continuous and non-decreasing on $\mathbb{R}$.

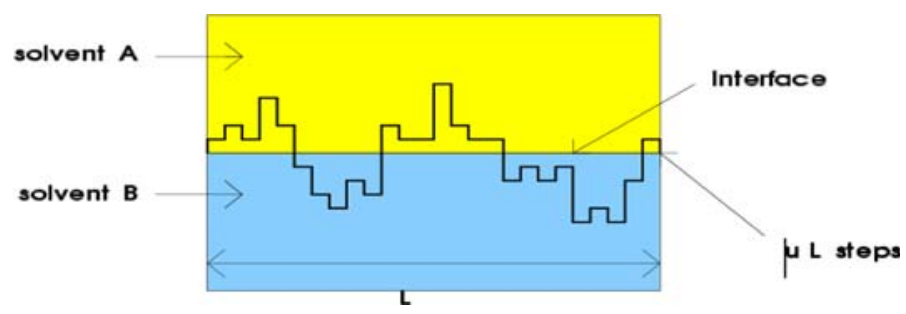

Fig. 7. Illustration of (2.3.2-2.3.3) for $c=\mu$ and $b=1$ 
The idea behind Lemma 2.3 .2 is that the copolymer follows the $A B$-interface over a distance $b L$ during $c L$ steps and then wanders away from the $A B$-interface to the diagonally opposite corner over a distance $(1-b) L$ during $(a-c) L$ steps. The optimal strategy is obtained by maximising over $b$ and $c$ (see Fig. 8). A similar expression holds for $\psi_{B A}$.

The key result behind the analysis of the critical curve in Fig. 4 is the following proposition, whose proof relies on Lemmas 2.3.1-2.3.3.

Proposition 2.3.4. ([7], Proposition 2.3.1)

Let $p \geq p_{c}$. Then $(\alpha, \beta) \in \mathcal{L}$ if and only if

$$
\sup _{\mu \geq 1} \mu\left[\phi^{\mathcal{I}}(\alpha, \beta ; \mu)-\frac{1}{2} \alpha-\frac{1}{2} \log 5\right]>\frac{1}{2} \log \frac{9}{5} .
$$

Note that $\frac{1}{2} \alpha+\frac{1}{2} \log 5$ is the free energy per step when the copolymer diagonally crosses an $A$-block. What Proposition 2.3.4 says is that for the copolymer in the emulsion to localize, the excess free energy of the copolymer along the interface must be sufficiently large to compensate for the loss of entropy of the copolymer coming from the fact that it must diagonally cross the block at a steeper angle (see Fig. 8).

We have

$$
\begin{aligned}
& \phi^{\mathcal{I}}(\mu) \geq \frac{1}{2} \alpha+\hat{\kappa}(\mu) \forall \mu>1, \\
& \phi^{\mathcal{I}}(\mu) \leq \alpha+\hat{\kappa}(\mu) \forall \mu \geq 1,
\end{aligned}
$$

where $\hat{\kappa}(\mu)$ is the entropy defined in (2.2.4). The upper bound and the gap in Lemma 2.2.2(iv) are responsible for the linear piece of the critical curve in Fig. 4. In analogy with Lemma 2.2.2, we further note that, for all $(\alpha, \beta) \in \mathbb{R}^{2}, \mu \mapsto \mu \phi^{\mathcal{I}}(\mu)$ is finite and concave on $[1, \infty)$, and hence is continuous on $(1, \infty)$. In the definition of $\phi^{\mathcal{I}}$ the interface belongs to solvent $B$ (see (2.3.3)), so that $\phi^{\mathcal{I}}(1)=\frac{1}{2} \beta$. Finally, by mimicking the proof of Lemma 2.4.1(i) below, we can show that $\lim _{\mu \downarrow 1} \phi^{\mathcal{I}}(\mu)=\frac{1}{2} \alpha$.

2.4. Tail behaviour of free energies for long paths. In this section we show that long paths wash out the effect of entropy. This will be needed later for compactification arguments.

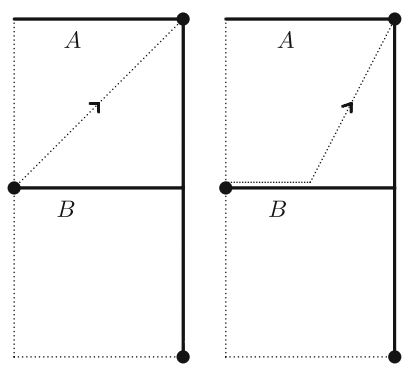

Fig. 8. Two possible strategies inside an $A B$-block: The path can either move straight across or move along the interface for awhile and then move across. Both strategies correspond to a coarse-grained step diagonally upwards as in Fig. 6 
Let $\mathbb{P}_{\mu L}^{\omega, \mathcal{I}}$ denote the law of the copolymer of length $\mu L$ in the single interface model with the energy shifted by $-\frac{\alpha}{2}$, i.e.,

$$
\mathbb{P}_{\mu L}^{\omega, \mathcal{I}}(\pi)=\frac{1}{Z_{\mu L, L}^{\omega, \mathcal{I}}} \exp \left[-H_{\mu L}^{\omega, \mathcal{I}}(\pi)\right], \quad \pi \in \mathcal{W}_{\mu L, L},
$$

with

$$
H_{\mu L}^{\omega, \mathcal{I}}(\pi)=-\sum_{i=1}^{\mu L}\left(-\alpha 1\left\{\omega_{i}=A\right\}+\beta 1\left\{\omega_{i}=B\right\}\right) 1\left\{\left(\pi_{i-1}, \pi_{i}\right) \leq 0\right\} .
$$

Let

$$
\begin{aligned}
\phi^{\mathcal{I}}(\mu) & =\phi^{\mathcal{I}}(\alpha, \beta ; \mu)=\lim _{L \rightarrow \infty} \phi_{\mu L}^{\omega, \mathcal{I}} \omega-a . s . \quad \text { with } \\
\phi_{\mu L}^{\omega, \mathcal{I}} & =\phi_{\mu L}^{\omega, \mathcal{I}}(\alpha, \beta)=\frac{1}{\mu L} \log Z_{\mu L, L}^{\omega, \mathcal{I}}
\end{aligned}
$$

(compare with (2.3.3)). Henceforth we adopt this shift, but we retain the same notation. The reader must keep this in mind throughout the sequel!

Lemma 2.4.1. For any $\beta_{0}>0$,

(i) $\lim _{\mu \rightarrow \infty} \phi^{\mathcal{I}}(\alpha, \beta ; \mu)=0$,

(ii) $\lim _{a \rightarrow \infty} \psi_{A B}(\alpha, \beta ; a)=0$, uniformly in $\alpha \geq \beta$ and $\beta \leq \beta_{0}$.

Proof. (i) Recall the definition of $\mathcal{W}_{\mu L, L}$ in Sect. 2.3. Abbreviate $\chi_{i}=1\left\{\omega_{i}=B\right\}-1$ $\left\{\omega_{i}=A\right\}$. Because $\alpha \geq \beta$ and $\beta \leq \beta_{0}$, we have

$$
\begin{aligned}
\phi^{\mathcal{I}}(\alpha, \beta ; \mu) & \leq \lim _{L \rightarrow \infty} \frac{1}{\mu L} \log \sum_{\pi \in \mathcal{W}_{\mu L, L}} \exp \left[\beta \sum_{i=1}^{\mu L} \chi_{i} 1\left\{\left(\pi_{i-1}, \pi_{i}\right) \leq 0\right\}\right] \\
& \leq \hat{\kappa}(\mu)+\beta_{0} \limsup _{L \rightarrow \infty} \frac{1}{\mu L} \max _{\pi \in \mathcal{W}_{\mu L, L}}\left\{\sum_{i=1}^{\mu L} \chi_{i} 1\left\{\left(\pi_{i-1}, \pi_{i}\right) \leq 0\right\}\right\} .
\end{aligned}
$$

We know from Lemma 2.2.2(iii) that $\lim _{\mu \rightarrow \infty} \hat{\kappa}(\mu)=0$. Therefore it suffices to show that for every $\varepsilon>0$ there exists a $\mu_{0}(\varepsilon) \geq 2$ such that

$$
\limsup _{L \rightarrow \infty} \frac{1}{\mu L} \max _{\pi \in \mathcal{W}_{\mu L, L}}\left\{\sum_{i=1}^{\mu L} \chi_{i} 1\left\{\left(\pi_{i-1}, \pi_{i}\right) \leq 0\right\}\right\} \leq \varepsilon \quad \omega-\text { a.s. } \quad \forall \mu \geq \mu_{0}(\varepsilon) .
$$

The random variables $\chi_{i}$ are i.i.d. \pm 1 with probability $\frac{1}{2}$. Let $I_{j}$ be the set of indices $i$ in the $j_{t h}$ excursion of $\pi$ on or below the interface. Then $\sum_{i=1}^{\mu L} \chi_{i} 1\left\{\left(\pi_{i-1}, \pi_{i}\right) \leq\right.$ $0\}=\sum_{j} \sum_{i \in I_{j}} \chi_{i}$. Let $\mathcal{F}_{\mu, L}$ denote the family of all possible sequences $I=\left(I_{j}\right)$ as 
$\pi$ runs over the set $\mathcal{W}_{\mu L, L}$, and write $|I|=\sum_{j}\left|I_{j}\right|$. For $0<\varepsilon \leq 1$, consider the quantity

$$
p_{\mu, L, \varepsilon}=\mathbb{P}\left(\exists I \in \mathcal{F}_{\mu, L}: \sum_{j} \sum_{i \in I_{j}} \chi_{i} \geq \varepsilon \mu L\right),
$$

where $\mathbb{P}$ denotes the probability law of $\omega$. By the exponential Markov inequality, there exists a $C>0$ such that

$$
\mathbb{P}\left(\sum_{i=1}^{N} \chi_{i} \geq \varepsilon R N\right) \leq e^{-C \varepsilon^{2} R N} \quad \forall N, R \geq 1, \forall 0<\varepsilon \leq 1 .
$$

Since $|I| \leq \mu L$ for all $I \in \mathcal{F}_{\mu, L}$, we can apply (2.4.7) with $N=|I|$ and $R=\mu L /|I|$ to estimate

$$
p_{\mu, L, \varepsilon} \leq \sum_{I \in \mathcal{F}_{\mu, L}} \mathbb{P}\left(\sum_{j} \sum_{i \in I_{j}} \chi_{i} \geq \varepsilon \frac{\mu L}{|I|}|I|\right) \leq\left|\mathcal{F}_{\mu, L}\right| e^{-C \varepsilon^{2} \mu L} .
$$

Since

$$
\left|\mathcal{F}_{\mu, L}\right| \leq\left(\begin{array}{c}
\mu L \\
L
\end{array}\right)^{2}=\exp [C(\mu) L+o(L)] \quad \text { as } L \rightarrow \infty,
$$

with $C(\mu) \sim \log \mu$ as $\mu \rightarrow \infty$, there exists a $C^{\prime}>0$ such that, for $\mu \geq 2$ and $L$ large enough, $\left|\mathcal{F}_{\mu, L}\right| \leq \exp \left[L C^{\prime} \log \mu\right]$ and hence $p_{\mu, L, \varepsilon} \leq \exp \left[L\left(C^{\prime} \log \mu-C \varepsilon^{2} \mu\right)\right]$. Thus, there exists a $\mu_{0}(\varepsilon) \geq 2$ such that for $\mu \geq \mu_{0}(\varepsilon)$,

$$
\sum_{L=1}^{\infty} p_{\mu, L, \varepsilon}<\infty
$$

The Borel-Cantelli lemma now allows us to assert that, $\omega$-a.s. for $\mu \geq \mu_{0}(\varepsilon)$ and $L$ large enough, the inequality $\sum_{j} \sum_{i \in I_{j}} \chi_{i} \leq \varepsilon \mu L$ holds uniformly in $I \in \mathcal{F}_{\mu, L}$. Hence (2.4.5) is true indeed.

(ii) This follows from a similar argument. The counterpart of Eq. (2.4.4) is (recall (2.2.1)$(2.2 .2))$

$$
\psi_{A B}(\alpha, \beta ; a) \leq \kappa(a, 1)+\beta_{0} \limsup _{L \rightarrow \infty} \frac{1}{a L} \max _{\pi \in N_{L}(a, 1)}\left\{\sum_{i=1}^{a L} \chi_{i} 1\left\{\left(\pi_{i-1}, \pi_{i}\right) \leq 0\right\}\right\} .
$$

Lemma 2.2.1(iii) implies that $\kappa(a, 1) \rightarrow 0$ as $a \rightarrow \infty$, while the proof that $\omega$-a.s. the second term in the r.h.s. of (2.4.11) tends to 0 is the same as in (i).

\section{Proof of Theorem 1.4.1}

In Sect. 3.1 we derive a proposition stating that the excursions away from the interface are exponentially tight in the localized phase. In Sect. 3.2 we use this proposition to prove Theorem 1.4.1. 
3.1. Tightness of excursions. We will call the triple $(\alpha, \beta, \mu) \in \operatorname{CONE} \times[1, \infty)$ weakly localized if (recall Proposition 2.3.4 and (2.4.1-2.4.3))

$$
\alpha \in\left(\alpha^{*}, \infty\right) \text { and } \sup _{\nu \geq 1} v\left[\phi^{\mathcal{I}}(\alpha, \beta ; \nu)-\varpi\right]=\mu\left[\phi^{\mathcal{I}}(\alpha, \beta ; \mu)-\varpi\right] \geq \varsigma
$$

with

$$
\varpi=\frac{1}{2} \log 5 \quad \text { and } \quad \varsigma=\frac{1}{2} \log \frac{9}{5} .
$$

Let $l_{\mu L}$ denote the number of strictly positive excursions in $\pi \in \mathcal{W}_{\mu L, L}$. For $k=1, \ldots, l_{\mu L}$, let $\tau_{k}$ denote the length of the $k_{t h}$ such excursion in $\pi$.

Proposition 3.1.1. Let $(\alpha, \beta, \mu)$ be a weakly localized triple. Then for every $C>0$ there exists an $M_{0}=M_{0}(C)$ such that for $M \geq M_{0}$,

$$
\lim _{L \rightarrow \infty} \mathbb{E}\left(\mathbb{P}_{\mu L}^{\omega, \mathcal{I}}\left(\sum_{k=1}^{l_{\mu L}} \tau_{k} 1\left\{\tau_{k} \geq M\right\} \geq C \mu L\right)\right)=0 .
$$

Proof. Along the way we need the following concentration inequality for the free energy of the single interface. Let $\phi_{\mu L}^{\omega, \mathcal{I}}=(1 / \mu L) \log Z_{\mu L, L}^{\omega, \mathcal{I}}$ (recall (2.3.3)).

Lemma 3.1.2. There exist $C_{1}, C_{2}>0$ such that for all $\varepsilon>0,(\alpha, \beta, \mu) \in \operatorname{CONE} \times[1, \infty)$ and $L \in \mathbb{N}$,

$$
\mathbb{P}\left(\left|\phi_{\mu L}^{\omega, \mathcal{I}}(\alpha, \beta)-\mathbb{E}\left(\phi_{\mu L}^{\omega, \mathcal{I}}(\alpha, \beta)\right)\right| \geq \varepsilon\right) \leq C_{1} \exp \left[-\varepsilon^{2} \mu L / C_{2}(\alpha+\beta)\right] .
$$

Proof. See Giacomin and Toninelli [6]. The argument for their single interface model readily extends to our single interface model.

Step 1. Throughout the proof, $(\alpha, \beta, \mu)$ is a weakly localized triple and $C \in(0,1)$. Fix $M$. For $\pi \in \mathcal{W}_{\mu L, L}$, we let

$$
K_{L}=K_{L}(\pi)=\left\{k \in\left\{1, \ldots, l_{\mu L}\right\}: \tau_{k} \geq M\right\} .
$$

We also define

$$
\begin{aligned}
& \tilde{\mathcal{W}}_{L}=\left\{\pi \in \mathcal{W}_{\mu L, L}: \sum_{k \in K_{L}} \tau_{k} \geq C \mu L\right\}, \\
& \mathcal{Q}_{L}=\{C \mu L, \ldots, \mu L\} \times\{1, \ldots, L\} \times\{1, \ldots, \mu L / M\} .
\end{aligned}
$$

Note that $\widetilde{\mathcal{W}}_{L}$ is the union of the events $\left(A_{s, r, t}\right)_{(s, r, t) \in \mathcal{Q}_{L}}$ with

$$
A_{s, r, t}=\left\{\sum_{k \in K_{L}} \tau_{k}=s\right\} \cap\left\{\sum_{k \in K_{L}} \tau_{k} / \mu_{k}=r\right\} \cap\left\{\left|K_{L}\right|=t\right\},
$$

where $\mu_{k}$ is the number of steps divided by the number of horizontal steps in the $k_{t h}$ strictly positive excursion. Let $v=\left(v_{k}^{1}, v_{k}^{2}\right)_{k \in K_{L}}$ denote the starting points and ending points of the successive positive excursions of length $\geq M$. If $V_{L}$ denotes all 
possible values of $v$, then $A_{s, r, t}$ is the union of the events $\left(A_{s, r, t}^{v}\right)_{v \in V_{L}}$. We will estimate $\mathbb{E}\left(\mathbb{P}_{\mu L}^{\omega, \mathcal{I}}\left(A_{s, r, t}^{v}\right)\right)$.

Step 2. We want to bound from above the quantity

$$
\mathbb{E}\left(\mathbb{P}_{\mu L}^{\omega, \mathcal{I}}\left(A_{s, r, t}^{v}\right)\right)=\mathbb{E}\left(\left(\sum_{\pi \in A_{s, r, t}^{v}} e^{-H_{\mu L}^{\omega, \mathcal{I}}(\pi)}\right) e^{-\mu L \phi_{\mu L}^{\omega, \mathcal{I}}}\right) .
$$

To that end, we concatenate the excursions of $\pi$ in $\left[v_{k-1}^{2}, v_{k}^{1}\right], k \in\{1, \ldots, t\}$, as follows. Since these excursions start and end at the interface, either with a horizontal step or with a vertical step up, we concatenate them by adding a strictly positive excursion of 3 steps between them. The latter has no effect on the Hamiltonian. We also concatenate the strictly positive excursions in $\left[v_{k}^{1}, v_{k}^{2}\right], k \in\{1, \ldots, t\}$, by adding 1 horizontal step between them. Thus, if we abbreviate $S_{1}=\mu L-s+3 t$ and $S_{2}=L-r+t$, and if we denote by $\omega_{v}$ the concatenation of the $\omega_{i}$ in $\left[v_{k-1}^{2}, v_{k}^{1}\right], k \in\{1, \ldots, t\}$, then we have

$$
\sum_{\pi \in A_{s, r, t}^{v}} e^{-H_{\mu L}^{\omega, \mathcal{I}}(\pi)} \leq \sum_{\pi \in \mathcal{W}_{S_{1}, S_{2}}} e^{-H_{S_{1}}^{\omega_{v}, \mathcal{I}}(\pi)} K(s+t, r+t),
$$

where $K(a, b)$ is the number of strictly positive excursions of length $a$ that make $b$ horizontal steps. A standard superadditivity argument gives

$$
K(s+t, r+t) \leq e^{(s+t) \hat{\kappa}\left(\frac{s+t}{r+t}\right)}
$$

with $\hat{\kappa}$ the entropy function defined in (2.2.4). Put $\hat{\mu}=S_{1} / S_{2}$. Then with (3.1.10) we can rewrite (3.1.9) as

$$
\sum_{\pi \in A_{s, r, t}^{v}} e^{-H_{\mu L}^{\omega, \mathcal{I}}(\pi)} \leq e^{S_{1} \phi_{\hat{\mu} S_{2}}^{\omega_{v}, \mathcal{I}}} e^{(s+t) \hat{\kappa}\left(\frac{s+t}{r+t}\right)} .
$$

At this stage, two cases need to be distinguished. Fix $\eta>0$.

Case $S_{1} \geq \eta L$. Let

$$
\begin{aligned}
& A_{1}=\left\{\phi_{\mu L}^{\omega, \mathcal{I}} \leq \mathbb{E}\left(\phi_{\mu L}^{\omega, \mathcal{I}}\right)-\varepsilon\right\}, \\
& A_{2}=\left\{\phi_{\hat{\mu} S_{2}}^{\omega_{v}, \mathcal{I}} \geq \mathbb{E}\left(\phi_{\hat{\mu} S_{2}}^{\omega_{v}, \mathcal{I}}\right)+\varepsilon\right\} .
\end{aligned}
$$

Since $\mu L \geq \hat{\mu} S_{2}=S_{1} \geq \eta L$, Lemma 3.1.2 gives the large deviation inequality

$$
\max \left\{\mathbb{P}\left(A_{1}\right), \mathbb{P}\left(A_{2}\right)\right\} \leq C_{1} \exp \left[-\varepsilon^{2} \eta L / C_{2}(\alpha+\beta)\right] .
$$

By superadditivity, we have $\mathbb{E}\left(\phi_{\hat{\mu} S_{2}}^{\omega_{v}, \mathcal{I}}\right) \leq \sup _{L \in \mathbb{N}} \mathbb{E}\left(\phi_{\hat{\mu} L}^{\omega_{v}, \mathcal{I}}\right)=\phi^{\mathcal{I}}(\hat{\mu})$. Moreover, for $L$ large enough, we have $\mathbb{E}\left(\phi_{\mu L}^{\omega, \mathcal{I}}\right) \geq \phi^{\mathcal{I}}(\mu)-\varepsilon$. Hence, it follows from (3.1.11-3.1.13) that

$$
\begin{aligned}
\mathbb{E}\left(\mathbb{P}_{\mu L}^{\omega, \mathcal{I}}\left(A_{s, r, t}^{v}\right)\right) & =\mathbb{E}\left(\left(\sum_{\pi \in A_{s, r, t}^{v}} e^{-H_{\mu L}^{\omega, \mathcal{I}}(\pi)}\right) e^{-\mu L \phi_{\mu L}^{\omega, \mathcal{I}}}\right) \\
& \leq \mathbb{P}\left(A_{1}\right)+\mathbb{P}\left(A_{2}\right)+\mathbb{E}\left(\left(\sum_{\pi \in A_{s, r, t}^{v}} e^{-H_{\mu L}^{\omega, \mathcal{I}}(\pi)}\right) e^{-\mu L \phi_{\mu L}^{\omega, \mathcal{I}}} 1_{A_{1}^{c} \cap A_{2}^{c}}\right) \\
& \leq 2 C_{1} e^{-\varepsilon^{2} \eta L / C_{2}(\alpha+\beta)}+e^{S_{1}\left(\phi^{\mathcal{I}}(\hat{\mu})+\varepsilon\right)} e^{-\mu L\left(\phi^{\mathcal{I}}(\mu)-2 \varepsilon\right)} e^{(s+t) \hat{\kappa}\left(\frac{s+t}{r+t}\right)} .
\end{aligned}
$$


Case $S_{1} \leq \eta L$. Note that, for $(\alpha, \beta) \in$ CONE, the trivial inequality $\phi_{\mu L}^{\omega, \mathcal{I}} \leq \alpha+\hat{\kappa}(\mu)$ (compare with (2.3.8)) and Lemma 2.2.2 (iii) are sufficient to assert that there exists an $R_{\alpha}>0$ such that $\phi_{\mu L}^{\omega, \mathcal{I}} \leq R_{\alpha}$ for all $\mu \geq 1, L \in \mathbb{N}$ and $\omega$. Therefore also $\phi_{\hat{\mu} S_{2}}^{\omega_{v}, \mathcal{I}} \leq R_{\alpha}$ for all $\hat{\mu} \geq 1, S_{2} \in \mathbb{N}$ and $\omega_{v}$, and so it follows from (3.1.11-3.1.13) that

$$
\begin{aligned}
\mathbb{E}\left(\mathbb{P}_{\mu L}^{\omega, \mathcal{I}}\left(A_{s, r, t}^{v}\right)\right) & =\mathbb{E}\left(\left(\sum_{\pi \in A_{s, r, t}^{v}} e^{-H_{\mu L}^{\omega, \mathcal{I}}(\pi)}\right) e^{-\mu L \phi_{\mu L}^{\omega, \mathcal{I}}}\right) \\
& =\mathbb{P}\left(A_{1}\right)+\mathbb{E}\left(\left(\sum_{\pi \in A_{s, r, t}^{v}} e^{-H_{\mu L}^{\omega, \mathcal{I}}(\pi)}\right) e^{-\mu L \phi_{\mu L}^{\omega, \mathcal{I}}} 1_{A_{1}^{c}}\right) \\
& \leq C_{1} e^{-\varepsilon^{2} \mu L / C_{2} \beta}+e^{S_{1} R_{\alpha}} e^{-\mu L\left(\phi^{\mathcal{I}}(\mu)-2 \varepsilon\right)} e^{(s+t) \hat{\kappa}\left(\frac{s+t}{r+t}\right)}
\end{aligned}
$$

Step 3. To bound the quantity $S_{1} \phi^{\mathcal{I}}(\hat{\mu})=S_{1} \phi^{\mathcal{I}}\left(S_{1} / S_{2}\right)$ in (3.1.14), we define $x=s / \mu L$ and $\tilde{\mu}=s / r$. Then $S_{1}=\mu L(1-x)+3 t$ and $S_{2}=L(1-x \mu / \tilde{\mu})+t$. Since $(\alpha, \beta, \mu)$ is a weakly localized triple (recall (3.1.1)), we have $S_{1} \phi^{\mathcal{I}}\left(S_{1} / S_{2}\right) \leq \mu S_{2} \phi^{\mathcal{I}}(\mu)+\varpi\left(S_{1}-\right.$ $\mu S_{2}$ ), with $\varpi$ given in (3.1.2). This can be further estimated by

$$
\begin{aligned}
S_{1} \phi^{\mathcal{I}}\left(S_{1} / S_{2}\right) \leq & \mu L \phi^{\mathcal{I}}(\mu)-\varpi x \mu L+x \frac{\mu^{2}}{\tilde{\mu}} L\left[\varpi-\phi^{\mathcal{I}}(\mu)\right] \\
& +t\left[\mu \phi^{\mathcal{I}}(\mu)+\varpi(3-\mu)\right] \\
\leq & \mu L \phi^{\mathcal{I}}(\mu)-\frac{5}{6} \varpi x \mu L,
\end{aligned}
$$

where we use that $\varpi-\phi^{\mathcal{I}}(\mu) \leq 0, t \leq \mu L / M$, and $M$ is large enough (by assumption). Next, let $\mu_{0}$ be such that $\hat{\kappa}(\nu) \leq \frac{\bar{\sigma}}{2}$ for all $v \geq \frac{\mu_{0}}{2}$ (which is possible by Lemma 2.2.2(iii)).

Case $\tilde{\mu} \geq \mu_{0}$. Since $s \geq c \mu L$ and $t \leq \mu L / M$, if $\tilde{\mu} \geq \mu_{0}$, then $(s+t) /(r+t) \geq$ $\tilde{\mu} /(1+t / r) \geq \frac{\mu_{0}}{2}$. Since $\bar{s}+t \leq x \mu L+\mu L / M$, it follows from (3.1.17) that for $M$ large enough,

$$
S_{1} \phi^{\mathcal{I}}\left(S_{1} / S_{2}\right)+(s+t) \hat{\kappa}\left(\frac{s+t}{r+t}\right) \leq \mu L \phi^{\mathcal{I}}(\mu)-\frac{1}{6} \varpi x \mu L
$$

Case $\tilde{\mu} \leq \mu_{0}$. For $\tilde{\mu}<\mu_{0}$, we first note that, by Lemma 2.2.2(iv) and (3.1.1), there exists a $z>0$ such that

$$
\sup _{y \geq 1} y[\hat{\kappa}(y)-\varpi]=\mu\left(\phi^{\mathcal{I}}(\mu)-\varpi\right)-z .
$$

Therefore, picking $y=(s+t) /(r+t)$ in (3.1.19), we get

$$
\begin{aligned}
(s+t) \hat{\kappa}\left(\frac{s+t}{r+t}\right) & \leq \mu(r+t) \phi^{\mathcal{I}}(\mu)+\varpi[(s+t)-\mu(r+t)]-z(r+t) \\
& \leq \mu r \phi^{\mathcal{I}}(\mu)+\varpi(s-\mu r)-z r+\frac{C^{\prime} L}{M} \\
& =x \frac{\mu^{2} L}{\tilde{\mu}} \phi^{\mathcal{I}}(\mu)+\varpi x \mu L\left(1-\frac{\mu}{\tilde{\mu}}\right)-z \frac{x \mu L}{\tilde{\mu}}+\frac{C^{\prime} L}{M},
\end{aligned}
$$


where $C^{\prime}=C^{\prime}(\mu)>0$ and the second line uses $t \leq \mu L / M$. Summing (3.1.16) and (3.1.20), we obtain that for $M$ large enough,

$$
S_{1} \phi^{\mathcal{I}}\left(S_{1} / S_{2}\right)+(s+t) \hat{\kappa}\left(\frac{s+t}{r+t}\right) \leq \mu L \phi^{\mathcal{I}}(\mu)-z \frac{x \mu L}{\tilde{\mu}}+\frac{C^{\prime} L}{M} .
$$

Since $x \geq C$ and $\tilde{\mu} \leq \mu_{0}$, we can choose $M$ large enough such that the r.h.s. of (3.1.21) is bounded from above by $\mu L \phi^{\mathcal{I}}(\mu)-\frac{z C}{2 \tilde{\mu}_{0}} \mu L$.

Setting $C_{3}=\inf \left\{z C / 2 \tilde{\mu_{0}}, \varpi C / 6\right\}$, we obtain that the r.h.s. of (3.1.18) and (3.1.21) are both bounded from above by $\mu L \phi^{\mathcal{I}}(\mu)-C_{3} \mu L$.

Step 4. In the case $S_{1} \geq \eta L,(3.1 .14)$ becomes

$$
\mathbb{E}\left(\mathbb{P}_{\mu L}^{\omega, \mathcal{I}}\left(A_{s, r, t}^{v}\right)\right) \leq 2 C_{1} e^{-\varepsilon^{2} \eta L / C_{2}(\alpha+\beta)}+e^{\mu L\left(-C_{3}+3 \varepsilon\right)},
$$

while in the case $S_{1} \leq \eta L$ we choose $\eta \leq C_{3} / 2 R_{\alpha}$, and (3.1.15) becomes

$$
\mathbb{E}\left(\mathbb{P}_{\mu L}^{\omega, \mathcal{I}}\left(A_{s, r, t}^{v}\right)\right) \leq C_{1} e^{-\varepsilon^{2} \mu L / C_{2}(\alpha+\beta)}+e^{\mu L\left(-\frac{1}{2} C_{3}+2 \varepsilon\right)} .
$$

Thus, there are $C_{4}, C_{5}>0$ such that, for $\varepsilon$ small enough,

$$
\mathbb{E}\left(\mathbb{P}_{\mu L}^{\omega, \mathcal{I}}\left(A_{s, r, t}^{v}\right)\right) \leq C_{4} e^{-C_{5} \mu L}
$$

Therefore it remains to estimate the number of possible values of $(s, r, t)$ and $v$. Since $(s, r, t) \in\{1, \ldots, \mu L\}^{3}$, there are at most $(\mu L)^{3}$ such triples. At fixed $t$, choosing $v$ amounts to choosing $t$ starting points and $t$ ending points for the excursions, which can be done in at most $\left(\begin{array}{c}\mu L \\ 2 t\end{array}\right) \leq\left(\begin{array}{c}\mu L \\ 2 \mu L / M\end{array}\right)$ ways when $M \geq 4$. By Stirling's formula there exists a $C^{\prime \prime}>0$ such that for all $M \geq 4$ and $L \in \mathbb{N}$,

$$
\left(\begin{array}{c}
\mu L \\
2 \mu L / M
\end{array}\right) \leq C^{\prime \prime} \sqrt{\mu L} e^{d(M) \mu L} \quad \text { with } \quad d(M)=-\frac{2}{M} \log \left(\frac{2}{M}\right)-\left(1-\frac{2}{M}\right) \log \left(1-\frac{2}{M}\right) .
$$

Since $\lim _{M \rightarrow \infty} d(M)=0$, we have $d(M) \leq C_{5} / 2$ for some $C_{5}>0$ and $M$ large enough. Therefore

$$
\sum_{(s, r, t) \in \mathcal{Q}_{L}} \sum_{v} \mathbb{E}\left(\mathbb{P}_{\mu L}^{\omega, \mathcal{I}}\left(A_{s, r, t}^{v}\right)\right) \leq C_{4} C^{\prime \prime}(\mu L)^{7 / 2} e^{-C_{5} \mu L / 2} .
$$

Since the 1.h.s. equals the expectation in (3.1.3), we have completed the proof.

3.2. Proof of Theorem 1.4.1. The proof uses Lemma 2.2.1 and Proposition 3.1.1.

Step 1. From Theorem 1.3.2(ii) we know that $\alpha \mapsto \beta_{c}(\alpha)$ is non-decreasing and converges to a finite limit $\beta^{*}$ as $\alpha \rightarrow \infty$. Equation (2.3.7), which gives a criterion for the localization of the copolymer at $A B$-interfaces, implies that

$$
\sup _{\mu \geq 1} \mu\left[\phi^{\mathcal{I}}\left(\alpha, \beta_{c}(\alpha) ; \mu\right)-\varpi\right]=\varsigma \quad \forall \alpha \geq 0
$$

with $\varpi, \varsigma$ defined in (3.1.2) (recall the energy shift made in (2.4.1-2.4.3)). Lemma 2.4.1 asserts that $\phi^{\mathcal{I}}\left(\alpha, \beta_{c}(\alpha) ; \mu\right)$ tends to zero as $\mu \rightarrow \infty$, uniformly in $\alpha \geq 0$. Since 
$\phi^{\mathcal{I}}\left(\alpha, \beta_{c}(\alpha) ; 1\right)=0$ for all $\alpha>0$ (the path lies in the interface), it follows that the supremum in (3.2.1) is attained at some $\mu_{\alpha}>1$. Therefore, if we can prove that

$$
\phi^{\mathcal{I}}\left(\alpha^{\prime}, \beta_{c}(\alpha) ; \mu_{\alpha}\right)>\phi^{\mathcal{I}}\left(\alpha, \beta_{c}(\alpha) ; \mu_{\alpha}\right) \quad \forall \alpha>\alpha^{\prime},
$$

then

$$
\begin{aligned}
\sup _{\mu \geq 1} \mu\left[\phi^{\mathcal{I}}\left(\alpha^{\prime}, \beta_{c}(\alpha) ; \mu\right)-\varpi\right] & \geq \mu_{\alpha}\left[\phi^{\mathcal{I}}\left(\alpha^{\prime}, \beta_{c}(\alpha) ; \mu_{\alpha}\right)-\varpi\right] \\
& >\mu_{\alpha}\left[\phi^{\mathcal{I}}\left(\alpha, \beta_{c}(\alpha) ; \mu_{\alpha}\right)-\varpi\right]=\varsigma,
\end{aligned}
$$

and hence $\beta_{c}(\alpha)>\beta_{c}\left(\alpha^{\prime}\right)$.

Step 2. Let $\alpha^{\prime}>\alpha$ and

$$
\begin{aligned}
D & =\phi^{\mathcal{I}}\left(\alpha^{\prime}, \beta_{c}(\alpha) ; \mu_{\alpha}\right)-\phi^{\mathcal{I}}\left(\alpha, \beta_{c}(\alpha) ; \mu_{\alpha}\right) \\
& =\lim _{L \rightarrow \infty} \frac{1}{\mu_{\alpha} L}\left[\log \sum_{\pi \in \mathcal{W}_{\mu_{\alpha} L, L}} e^{-H_{\mu_{\alpha} L}^{\omega, \mathcal{I}}\left(\alpha^{\prime}, \beta_{c}(\alpha) ; \pi\right)}-\log \sum_{\pi \in \mathcal{W}_{\mu_{\alpha} L, L}} e^{-H_{\mu_{\alpha} L}^{\omega, \mathcal{I}}\left(\alpha, \beta_{c}(\alpha) ; \pi\right)}\right] \\
& =\lim _{L \rightarrow \infty} \frac{1}{\mu_{\alpha} L} \log \mathbb{E}_{\mu_{\alpha} L}^{\omega, \mathcal{I}}\left(\exp \left[\left(\alpha-\alpha^{\prime}\right) \sum_{i=1}^{\mu_{\alpha} L} 1\left\{\omega_{i}=A,\left(\pi_{i-1}, \pi_{i}\right) \leq 0\right\}\right]\right),
\end{aligned}
$$

where the expectation is w.r.t. the law of the copolymer with parameters $\alpha$ and $\beta_{c}(\alpha)$, which are both suppressed from the notation. For $\varepsilon>0$, let $A_{\varepsilon, L}=\left\{\pi: \sum_{i=1}^{\mu_{\alpha} L} 1\left\{\omega_{i}=\right.\right.$ $\left.\left.A,\left(\pi_{i-1}, \pi_{i}\right) \leq 0\right\} \geq \varepsilon \mu_{\alpha} L\right\}$. Then we may estimate

$$
D \geq \limsup _{L \rightarrow \infty} \frac{1}{\mu_{\alpha} L} \log \left[e^{\left(\alpha-\alpha^{\prime}\right) \varepsilon \mu_{\alpha} L} \mathbb{P}_{\mu_{\alpha} L}^{\omega, \mathcal{I}}\left(A_{\varepsilon, L}\right)+\mathbb{P}_{\mu_{\alpha} L}^{\omega, \mathcal{I}}\left(\left[A_{\varepsilon, L}\right]^{c}\right)\right] .
$$

We will prove that, for $\varepsilon$ small enough, there is a subsequence $\left(L_{m}\right)_{m \in \mathbb{N}}$ such that $\lim _{m \rightarrow \infty} \mathbb{P}_{\mu_{\alpha} L_{m}}^{\omega, \mathcal{I}}\left(\left[A_{\varepsilon, L_{m}}\right]^{c}\right)=0 \omega$-a.s. This will imply that $D \geq\left(\alpha-\alpha^{\prime}\right) \varepsilon$ and complete the proof.

Step 3. We recall that $l_{\mu_{\alpha} L}$ denotes the number of strictly positive excursions in $\pi \in \mathcal{W}_{\mu_{\alpha} L, L}$. By Proposition 3.1.1, w-a.s., $\mathbb{P}_{\mu_{\alpha} L}^{\omega, \mathcal{I}}\left(\sum_{k=1}^{l_{\mu_{\alpha} L}} \tau_{k} 1\left\{\tau_{k} \geq M\right\} \geq C \mu_{\alpha} L\right)$ tends to zero as $L \rightarrow \infty$ along a subsequence. Moreover, $\omega$-a.s., $\sum_{i=1}^{\mu_{\alpha} L} 1\left\{\omega_{i}=A\right\} \geq$ $\frac{1}{2} \mu_{\alpha} L-C \mu_{\alpha} L$ for $L$ large enough. Thus, putting $s=\frac{1}{2}-2 C-\varepsilon$, for $L$ large enough we have the inclusion

$$
\begin{aligned}
& {\left[A_{\varepsilon, L}\right]^{c} \subset\left\{\sum_{k=1}^{l_{\mu_{\alpha} L}} \tau_{k} 1\left\{\tau_{k} \geq M\right\} \geq C \mu_{\alpha} L\right\}} \\
& \cup\left\{\left\{\sum_{i=1}^{\mu_{\alpha} L} 1\left\{\omega_{i}=A\right\} 1\left\{\Theta_{i}^{M}=1\right\} \geq s \mu_{\alpha} L\right\} \cap\left[A_{\varepsilon, L}\right]^{c}\right\},
\end{aligned}
$$

where $\Theta_{i}^{M}$ is the indicator of the event the $i_{t h}$ step lies in a strictly positive excursion of length $\leq M$. 
From now on we fix $C=\frac{1}{8}$ and $\varepsilon \leq \frac{1}{8}$, implying that $s \geq \frac{1}{8}$. We also fix $M$ such that Proposition 3.1.1 holds for $C=\frac{1}{8}$. The proof will be completed once we show that

$$
\lim _{L \rightarrow \infty} \mathbb{P}_{\mu_{\alpha} L}^{\omega, \mathcal{I}}\left(B_{\varepsilon, L}\right)=0 \quad \omega-\text { a.s. }
$$

where

$$
B_{\varepsilon, L}=\left\{\pi: \sum_{i=1}^{\mu_{\alpha} L} 1\left\{\omega_{i}=A\right\} 1\left\{\Theta_{i}^{M}=1\right\} \geq s \mu_{\alpha} L\right\} \cap\left[A_{\varepsilon, L}\right]^{c} .
$$

Each path of $B_{\varepsilon, L}$ puts at least $s \mu_{\alpha} L$ monomers labelled by $A$ in strictly positive excursions of length $\leq M$ and at most $\varepsilon \mu_{\alpha} L$ monomers labelled by $A$ in non-positive excursions.

Step 4. For $\pi \in B_{\varepsilon, L}$, let $\mathcal{E}_{L}(\pi)$ label the excursions of $\pi$ that are strictly positive, have length $\leq M$ and contain at least 1 monomer labelled by $A$. Abbreviate $r_{L}(\pi)=$ $\left|\mathcal{E}_{L}(\pi)\right| \geq s \mu_{\alpha} L / M$. Partition $\mathcal{E}_{L}(\pi)$ into two parts:

- $\mathcal{E}_{L}^{1}(\pi)$ : those excursions whose preceding and subsequent non-positive excursions do not contain an $A$.

- $\mathcal{E}_{L}^{2}(\pi)$ : those excursions whose preceding and/or subsequent non-positive excursions contain an $A$.

The total number of non-positive excursions containing an $A$ is bounded from above by $\varepsilon \mu_{\alpha} L$. Since a non-positive excursion can be at most once preceding and once subsequent, we have $\left|\mathcal{E}_{L}^{1}(\pi)\right| \geq(s / M-2 \varepsilon) \mu_{\alpha} L$. We will discard the excursions in $\mathcal{E}_{L}^{2}(\pi)$. Morover, to avoid overlap, we will keep from $\mathcal{E}_{L}^{1}(\pi)$ only half of the excursions. Call the remainder $\tilde{\mathcal{E}}_{L}^{1}(\pi)$, and abbreviate $\tilde{r}_{L}(\pi)=\left|\tilde{\mathcal{E}}_{L}^{1}(\pi)\right|$. Then $\tilde{r}_{L}(\pi) \geq r \mu_{\alpha} L$ with $r=(s / 2 M-\varepsilon) \mu_{\alpha} L$.

Next, for $\pi \in B_{\varepsilon, L}$, let $\chi(\pi)$ denote the partition of $\left\{1, \ldots, \mu_{\alpha} L\right\}$ into $2 \tilde{r}_{L}(\pi)+$ 1 intervals, i.e., $\left(I_{t}\right)_{t=0}^{2 \tilde{r}_{L}}$ with $I_{2(j-1)+1}, j \in\left\{1,2, \ldots, \tilde{r}_{L}\right\}$, the interval occupied by the $j_{t h}$ excursion of $\tilde{\mathcal{E}}_{L}^{1}(\pi)$ and its preceding and subsequent non-positive excursions. The partition $\chi(\pi)$ also contains $2 \tilde{r}_{L}+1$ integers $\left(i_{t}\right)_{t=0}^{2 \tilde{r}_{L}}$ with $i_{t}, i \in\left\{0,1, \ldots, 2 \tilde{r}_{L}\right\}$, the number of horizontal steps the path $\pi$ makes in $I_{t}$.

Let $K_{L}^{\omega}$ be the set of possible outcomes of $\chi(\pi)$ as $\pi$ runs over $B_{\varepsilon, L}$. For $\chi \in K_{L}^{\omega}$, let $t(\chi)$ denote the family of possible paths over the even intervals $I_{0}, I_{2}, \ldots, I_{2} \tilde{r}(\chi)$. The paths of $t(\chi)$ do not put more than $\varepsilon \mu_{\alpha} L$ monomers of type $A$ on or below the interface, put exactly one excursion of type 1 in each interval $I_{2 j}, j \in\{1, \ldots, 2 \tilde{r}(\chi)\}$, no excursion of type 1 in $I_{0}$ and at most one excursion in $I_{2 \tilde{r}}(\chi)$. For $j \in\{1, \ldots, \tilde{r}(\chi)\}$, let $t_{j}(\chi)$ be the set of paths on $I_{2 j-1}$ that make $i_{2 j-1}$ horizontal steps, perform exactly one excursion of type 1, and have their preceding and subsequent non-positive excursions without an $A$. Then we have the formula

$$
\mathbb{P}_{\mu_{\alpha} L}^{\omega, \mathcal{I}}\left(B_{\varepsilon, L}\right)=\frac{\sum_{\chi \in K_{L}^{\omega}}\left[\left(\sum_{\pi^{\prime} \in t(\chi)} e^{-H^{\omega, \mathcal{I}}\left(\pi^{\prime}\right)}\right) \prod_{j=1}^{\tilde{r}(\chi)}\left(\sum_{\pi_{j} \in t_{j}(\chi)} e^{-H^{\omega, \mathcal{I}}\left(\pi_{j}\right)}\right)\right]}{\sum_{\pi \in \mathcal{W}_{\mu_{\alpha} L, L}} e^{-H^{\omega, \mathcal{I}}(\pi)}} .
$$

Step 5. For $j \in\{1, \ldots, \tilde{r}(\chi)\}$, let $s_{j}(\chi)$ be the set of non-positive excursions of $\left|I_{2 j-1}\right|$ steps of which $i_{2 j-1}$ are horizontal. Then we may estimate 


$$
\begin{aligned}
& \mathbb{P}_{\mu_{\alpha} L}^{\omega, \mathcal{I}}\left(B_{\varepsilon, L}\right) \leq \varepsilon \mu_{\alpha} L\left(\begin{array}{c}
\mu_{\alpha} L \\
\varepsilon \mu_{\alpha} L
\end{array}\right) \\
& \times \frac{\sum_{\chi \in K_{L}^{\omega}}\left[\left(\sum_{\pi^{\prime} \in t(\chi)} e^{-H^{\omega, \mathcal{I}}\left(\pi^{\prime}\right)}\right) \prod_{j=1}^{\tilde{r}(\chi)}\left(\sum_{\pi_{j} \in t_{j}(\chi)} e^{-H^{\omega, \mathcal{I}}\left(\pi_{j}\right)}\right)\right]}{\sum_{\chi \in K_{L}^{\omega}}\left[\left(\sum_{\pi^{\prime} \in t(\chi)} e^{-H^{\omega, \mathcal{I}}\left(\pi^{\prime}\right)}\right) \prod_{j=1}^{\tilde{r}(\chi)}\left(\sum_{\pi_{j} \in t_{j}(\chi)} e^{-H^{\omega, \mathcal{I}}\left(\pi_{j}\right)}+\sum_{\pi_{j} \in s_{j}(\chi)} e^{-H^{\omega, \mathcal{I}}\left(\pi_{j}\right)}\right)\right]} .
\end{aligned}
$$

Here, the prefactor comes from the fact that a path with more than one non-positive excursion containing an $A$ may be associated with more than one family $(\chi, t(\chi))$ in the sum in the denominator of (3.2.9). However, a path $t(\chi)$ cannot have more than $\varepsilon \mu_{\alpha} L$ excursions of such type. Since the number of excursions is bounded from above by $\mu_{\alpha} L$, we can assert that each path can appear at most $\varepsilon \mu_{\alpha} L\left(\begin{array}{c}\mu_{\alpha} L \\ \varepsilon \mu_{\alpha} L\end{array}\right)$ times in the denominator.

At this stage it suffices to show that there exists a $C>0$, depending only on $\alpha, \alpha^{\prime}$ and $M$, such that for all $\chi \in K_{L}^{\omega}$ and $j \in\{1, \ldots, \tilde{r}(\chi)\}$,

$$
\sum_{\pi_{j} \in s_{j}(\chi)} e^{-H^{\omega, \mathcal{I}}\left(\pi_{j}\right)} \geq C \sum_{\pi_{j} \in t_{j}(\chi)} e^{-H^{\omega, \mathcal{I}}\left(\pi_{j}\right)} .
$$

Indeed, since $r \geq \mu_{\alpha} L$ this yields, via (3.2.10),

$$
\mathbb{P}_{\mu_{\alpha} L}^{\omega, \mathcal{I}}\left(B_{\varepsilon, L}\right) \leq \varepsilon \mu_{\alpha} L\left(\begin{array}{c}
\mu_{\alpha} L \\
\varepsilon \mu_{\alpha} L
\end{array}\right)(1+C)^{-r \mu_{\alpha} L} .
$$

For $\varepsilon$ small enough the r.h.s. of (3.2.12) tends to zero as $L \rightarrow \infty$ because $C>0$, implying (3.2.7) as desired.

Step 6. To prove (3.2.12), we note that, since the paths of $s_{j}(\chi)$ stay in the lower halfplane, their Hamiltonian is a constant, namely, $H^{\omega, \mathcal{I}}\left(s_{j}(\chi)\right)=\sum_{i \in I_{j}}\left(\alpha 1\left\{\omega_{i}=A\right\}-\beta 1\left\{\omega_{i}=\right.\right.$ $B\}$ ) (recall (2.4.2)). A path of $t_{j}(\chi)$ puts at most $M$ steps of $I_{j}$ in the upper halfplane, and so $\pi_{j} \in t_{j}(\chi)$ implies $H^{\omega, \mathcal{I}}\left(\pi_{j}\right) \geq H^{\omega, \mathcal{I}}\left(s_{j}(\chi)\right)-\alpha M$. It therefore remains to compare the cardinalities of $s_{j}(\chi)$ and $t_{j}(\chi)$. The number of strictly positive excursions of length $\leq M$ is some integer, denoted by $\sharp(M)$. Moreover, on $I_{j}$ the possible starting points of the excursion of type 1 are at most $M$. Indeed, the excursion has to contain all the $\omega_{i}$ of $I_{j}$ that are equal to $A$, and hence it must start less than $M$ steps to the left of the leftmost $i \in I_{j}$ such that $\omega_{i}=A$. Thus, we have at most $M \sharp(M)$ possible excursions of type 1 in $I_{j}$ (if we take into account their starting point). Next, we note that by fixing the starting point and the shape of the excursions of type 1, we can create an injection from $t_{j}(\chi)$ to $s_{j}(\chi)$ as follows (see Fig. 9). If $2 r$ is the number of vertical steps in the fixed excursion of type 1 , then we associate with each path of $t_{j}(\chi)$ a path of $s_{j}(\chi)$ that begins with $r$ vertical steps down before performing the preceding non-positive excursion, next makes $s$ horizontal steps, where $s$ is the number of horizontal steps in the excursion of type 1, next performs the subsequent non-positive excursion, and afterwards returns to the interface with $r$ vertical steps.

We conclude that $\left|s_{j}(\chi)\right| \geq\left|t_{j}(\chi)\right| / M h(M)$, which allows us to estimate

$$
\begin{aligned}
\sum_{\pi_{j} \in s_{j}(\chi)} e^{-H^{\omega, \mathcal{I}}\left(\pi_{j}\right)} & =\left|s_{j}(\chi)\right| e^{-H^{\omega, \mathcal{I}}\left(s_{j}(\chi)\right)} \\
& \geq \frac{\left|t_{j}(\chi)\right|}{M \sharp(M)} e^{-H^{\omega, \mathcal{I}}\left(s_{j}(\chi)\right)}=C \sum_{\pi_{j} \in t_{j}(\chi)} e^{-H^{\omega, \mathcal{I}}\left(\pi_{j}\right)}
\end{aligned}
$$

with $C=e^{-\alpha M} / M h(M)$, proving (3.2.11). 

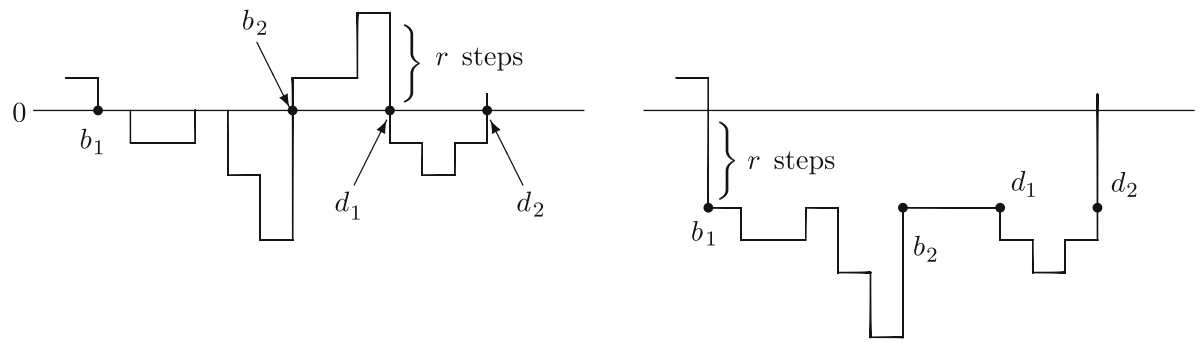

Fig. 9. Injection from $t_{j}(\chi)$ to $s_{j}(\chi)$. Here, $\left(b_{1}, b_{2}\right)$ and $\left(d_{1}, d_{2}\right)$ label the endpoints of the preceding and subsequent non-positive excursions

\section{Proof of Theorem 1.4.2}

Section 4.1 states two propositions providing the lower, respectively, upper bound for $f$ near the critical curve. These two propositions are proved in Sects. 4.3 and 4.4, respectively, and together yield Theorem 1.4.2. Section 4.2 contains several lemmas about the maximisers of the variational problem for $\psi_{A B}$, which are needed in the proofs.

4.1. Lower and upper bounds on the free energy. Recall (2.4.2). Fix $p \geq p_{c}$, $\alpha \in\left(\alpha^{*}, \infty\right)$ and $\delta_{0}>0$ small enough (depending on $p$ and $\alpha$ ). Abbreviate $I_{0}=\left(0, \delta_{0}\right] \cap\left(0, \alpha-\beta_{c}(\alpha)\right]$, and for $\delta \in I_{0}$ define

$$
\begin{aligned}
& \psi_{k l}(a, \delta)=\psi_{k l}\left(\alpha, \beta_{c}(\alpha)+\delta ; a\right), \quad a \geq 2, \\
& \phi^{\mathcal{I}}(\mu, \delta)=\phi^{\mathcal{I}}\left(\alpha, \beta_{c}(\alpha)+\delta ; \mu\right), \quad \mu \geq 1,
\end{aligned}
$$

and

$$
T_{\alpha}(\delta)=f\left(\alpha, \beta_{c}(\alpha)+\delta ; p\right)-f\left(\alpha, \beta_{c}(\alpha) ; p\right) .
$$

Proposition 4.1.1. There exists a $C_{1}>0$ such that

$$
T_{\alpha}(\delta) \geq C_{1} \delta^{2} \quad \forall \delta \in I_{0} .
$$

Proposition 4.1.2. There exists a $C_{2}<\infty$ such that

$$
T_{\alpha}(\delta) \leq C_{2} \delta^{2} \quad \forall \delta \in I_{0} .
$$

4.2. Maximisers of the block pair free energy. Lemmas 4.2.1-4.2.6 below are elementary assertions about the existence and the limiting behaviour of the maximisers in the variational expression for $\psi_{A B}$ in (2.3.6). These lemmas will be needed in the proof of Propositions 4.1.1-4.1.2 in Sects. 4.3-4.4.

Step 1. We first show that $a \mapsto \psi_{A B}(a, \delta)$ has a maximiser for $\delta$ small enough.

Lemma 4.2.1. For every $\delta_{0}>0$ there exists an $a_{0}>2$ such that, for every $\alpha>\alpha^{*}$ and $\delta \in I_{0}(\alpha)$, there exists an $a_{\alpha}(\delta) \in\left(2, a_{0}\right]$ satisfying

$$
\sup _{a \geq 2} \psi_{A B}(a, \delta)=\psi_{A B}\left(a_{\alpha}(\delta), \delta\right) .
$$


Proof. Recall (4.1.1). In Lemma 2.4.1 we showed that, for every $\beta_{0}>0, \psi_{A B}(a, \alpha, \beta)$ tends to zero as $a \rightarrow \infty$ uniformly in $\alpha \geq \beta$ and $\beta \leq \beta_{0}$. Since $\beta_{c}(\alpha) \leq \beta^{*}$ for all $\alpha \geq 0$, there therefore exists an $a_{0}>2$ such that $\psi_{A B}(a, \delta)<\kappa\left(a^{*}, 1\right)$ for all $a \geq a_{0}$, $\alpha>\alpha^{*}$ and $\delta \in I_{0}(\alpha)$. By [7], Theorem 1.4.2, we have $\sup _{a \geq 2} \psi_{A, B}(a, \delta)>\kappa\left(a^{*}, 1\right)$ for all $\delta>0$ and $\alpha>\alpha *$. This implies

$$
\sup _{a \geq 2} \psi_{A B}(a, \delta)=\sup _{2 \leq a \leq a_{0}} \psi_{A B}(a, \delta) \quad \forall \alpha>\alpha^{*}, \delta \in I_{0}(\alpha) .
$$

For $\delta$ fixed, $a \mapsto \psi_{A B}(a, \delta)$ is continuous on $[2, \infty)$ and $\psi_{A B}(2, \delta)=0$. Therefore there exists an $a_{\alpha}(\delta) \in\left(2, a_{0}\right]$ such that the 1.h.s. of (4.2.2) is equal to $\psi_{A, B}\left(a_{\alpha}(\delta), \delta\right)$.

Step 2. Let

$$
\mathcal{Q}_{\delta, \mu_{0}}^{\alpha}=\left\{(c, \mu): 0 \leq c \leq \mu, \mu \geq \mu_{0}, a_{\alpha}(\delta)-c \geq 2-c / \mu\right\}
$$

and

$$
H(c, a, \mu, \delta)=\frac{1}{a}\left[c \phi^{\mathcal{I}}(\mu, \delta)+(a-c) \kappa(a-c, 1-c / \mu)\right] .
$$

Then, by Lemma 2.2.1(ii), we can assert that there exists a unique pair $\left(c_{\alpha}(\delta), \mu_{\alpha}(\delta)\right) \in$ $\mathcal{Q}_{\delta, 1}^{\alpha}$ satisfying $\psi_{A B}\left(a_{\alpha}(\delta), \delta\right)=H\left(c_{\alpha}(\delta), a_{\alpha}(\delta), \mu_{\alpha}(\delta), \delta\right)$.

Lemma 4.2.2. For every $\delta_{0}>0$ there exists a $\mu_{0}>1$ such that $\left(c_{\alpha}(\delta), \mu_{\alpha}(\delta)\right) \in$ $\mathcal{Q}_{\delta, 1}^{\alpha} \backslash \mathcal{Q}_{\delta, \mu_{0}}^{\alpha}$ for all $\alpha>\alpha^{*}$ and $\delta \in I_{0}(\alpha)$.

Proof. Prior to (4.2.2) we noted that $\psi_{A B}\left(a_{\alpha}(\delta), \delta\right)>\kappa\left(a^{*}, 1\right)$. We will show that there exists a $\mu_{0}>1$ such that $H\left(c, a_{\alpha}(\delta), \mu, \delta\right) \leq \kappa\left(a^{*}, 1\right)$ for all $\alpha>\alpha^{*}, \delta \in I_{0}(\alpha)$ and $(c, \mu) \in \mathcal{Q}_{\delta, \mu_{0}}^{\alpha}$. This goes as follows. In Lemma 2.4.1(i) we showed that $\phi^{\mathcal{I}}(\mu, \delta)$ tends to zero as $\mu \rightarrow \infty$, uniformly in $\alpha>\alpha^{*}$ and $\delta \in I_{0}(\alpha)$. Therefore there exists a $\mu_{0}>1$ such that $\phi^{\mathcal{I}}(\mu, \delta)<\frac{1}{2} \kappa\left(a^{*}, 1\right)$ for all $\mu \geq \mu_{0}, \alpha>\alpha^{*}$ and $\delta \in I_{0}(\alpha)$.

Lemma 4.2.3. There exists an $M>0$, depending on $a_{0}$, such that $\kappa(a, b) \leq \kappa\left(a^{*}, 1\right)+$ $M(1-b)$ for all $(a, b) \in \operatorname{DOM}\left(\right.$ recall (2.2.1)) satisfying $a \leq a_{0}$ and $\frac{1}{2} \leq \bar{b} \leq 1$.

Proof. This is easily proved via Lemma 2.2.1(ii), which says that $(a, b) \mapsto \kappa(a, b)$ is analytic on the interior of DOM, and the equality $\kappa(a, a-1)=0$ for all $a \geq 2$.

We now choose $\mu_{0}$ large enough so that $\mu>2 a_{0}$ and $M a_{0} / \mu \leq \frac{1}{2} \kappa\left(a^{*}, 1\right)$. Thus, for $(c, \mu) \in \mathcal{Q}_{\delta, \mu_{0}}^{\alpha}$ we have $c / \mu \leq a_{0} / \mu_{0} \leq \frac{1}{2}$, which entails $\frac{1}{2} \leq 1-c / \mu \leq 1$. Therefore, $\left(a_{\alpha}(\delta)-c, 1-c / \mu\right)$ satisfies the assumptions of Lemma 4.2.3 and

$$
\begin{aligned}
H\left(c, a_{\alpha}(\delta), \mu, \delta\right) & \leq \frac{1}{a_{\alpha}(\delta)}\left[c \frac{1}{2} \kappa\left(a^{*}, 1\right)+\left(a_{\alpha}(\delta)-c\right)\left(\kappa\left(a^{*}, 1\right)+M c / \mu\right)\right] \\
& \leq \kappa\left(a^{*}, 1\right)+\frac{1}{a_{\alpha}(\delta)} c\left[M a_{0} / \mu-\frac{1}{2} \kappa\left(a^{*}, 1\right)\right] \leq \kappa\left(a^{*}, 1\right) .
\end{aligned}
$$


Step 3. We next show that $a \mapsto \psi_{A B}(a, 0)$ has a unique maximiser.

Lemma 4.2.4. For every $\alpha \geq \alpha^{*}, \sup _{a \geq 2} \psi_{A B}(a, 0)=\kappa\left(a^{*}, 1\right)$ and is achieved uniquely at $a=a^{*}$. Consequently, for $\alpha \geq \alpha^{*}$ and $\beta=\beta_{c}(\alpha)$, the supremum in (2.3.6) is achieved uniquely at $c=0$.

Proof. Since $\left(\alpha, \beta_{c}(\alpha)\right) \in \mathcal{L}$, [7], Theorem 1.4.2, tells us that $\sup _{a \geq 2} \psi_{A B}(a, 0) \leq$ $\kappa\left(a^{*}, 1\right)$. Moreover, $\psi_{A B}\left(a^{*}, 0\right) \geq \kappa\left(a^{*}, 1\right)$, and therefore

$$
\sup _{a \geq 2} \psi_{A B}(a, 0)=\kappa\left(a^{*}, 1\right)=\psi_{A B}\left(a^{*}, 0\right) .
$$

Now, pick $a \geq 2$ such that $\psi_{A B}(a, 0)=\kappa\left(a^{*}, 1\right)$ and recall that $\operatorname{DOM}(a)$ in $(2.3 .4)$ is the domain of the variational problem for $\psi_{A B}(a, 0)$. We argue by contradiction. Suppose that there exist $c, b>0$ such that $(c, b) \in \operatorname{DOM}(a)$ and

$$
\psi_{A B}(a, 0)=\kappa\left(a^{*}, 1\right)=\frac{1}{a}\left[c \phi^{\mathcal{I}}(c / b, 0)+(a-c) \kappa(a-c, 1-b)\right] .
$$

Then

$$
\frac{1}{a}\left\{(c / b)\left[\phi^{\mathcal{I}}(c / b, 0)-\kappa\left(a^{*}, 1\right)\right]-(a / b-c / b)\left[\kappa\left(a^{*}, 1\right)-\kappa(a-c, 1-b)\right]\right\}=0 .
$$

However, $(c / b)\left[\phi^{\mathcal{I}}(c / b, 0)-\kappa\left(a^{*}, 1\right)\right] \leq \varsigma$ by Proposition 2.3.4. Moreover, by [7], Eq. (2.3.3), we have

$$
g(v)=v\left[\kappa\left(a^{*}, 1\right)-\sup _{2 /(v+1) \leq b \leq 1} \kappa(b v, 1-b)\right]>\varsigma \quad \forall v \geq 1 .
$$

Pick $v=(a-c) / b$ to make the 1.h.s. of (4.2.8) strictly negative. Then the equality in (4.2.8) cannot occur with $b>0$ and $c>0$. Consequently, the only way to obtain (4.2.8) is to take $c=0$ and $a=a^{*}$.

Step 4. Fix $\alpha>\alpha^{*}$ and $\delta_{0}>0$. For $\delta \in I_{0}(\alpha)$, the quantity $a_{\alpha}(\delta)$ may not be unique, which is why from now on we take its minimum value. We next prove that $\left(a_{\alpha}(\delta), c_{\alpha}(\delta)\right)$ tends to $\left(a^{*}, 0\right)$ as $\delta \downarrow 0$. In what follows, $\left(\delta_{n}\right)_{n \in \mathbb{N}}$ is a sequence in $I_{0}(\alpha)$ such that $\lim _{n \rightarrow \infty} \delta_{n}=0$.

Lemma 4.2.5. Let $\left(a_{n}\right)_{n \in \mathbb{N}}$ and $\left(\mu_{n}\right)_{n \in \mathbb{N}}$ be such that $\lim _{n \rightarrow \infty} a_{n}=a \geq 2$ and $\lim _{n \rightarrow \infty} \mu_{n}=\mu \geq 1$. Then $\lim _{n \rightarrow \infty} \psi_{A B}\left(a_{n}, \delta_{n}\right)=\psi_{A, B}(a, 0)$ and $\lim _{n \rightarrow \infty} \phi^{\mathcal{I}}$ $\left(\mu_{n}, \delta_{n}\right)=\phi^{\mathcal{I}}(\mu, 0)$.

Proof. A simple computation gives that $\psi_{A B}(a, \delta)-\psi_{A B}(a, 0) \leq \delta$ for all $a \geq 2$ (recall (4.1.1)). This allows us to write the inequality

$$
\begin{aligned}
\left|\psi_{A B}\left(a_{n}, \delta_{n}\right)-\psi_{A B}(a, 0)\right| & =\left|\psi_{A B}\left(a_{n}, \delta_{n}\right)-\psi_{A B}\left(a_{n}, 0\right)\right|+\left|\psi_{A B}\left(a_{n}, 0\right)-\psi_{A B}(a, 0)\right| \\
& \leq \delta_{n}+\left|\psi_{A B}\left(a_{n}, 0\right)-\psi_{A B}(a, 0)\right| .
\end{aligned}
$$

Since $a \mapsto \psi_{A, B}(a, 0)$ is continuous (recall Lemma 2.3.3(i)), the r.h.s. of (4.2.10) tends to zero as $n \rightarrow \infty$. This yields the claim for $\psi_{A B}$. The same proof gives the claim for $\phi^{\mathcal{I}}$.

Step 5. Finally, we obtain the convergence of $a_{\alpha}(\delta)$ and $c_{\alpha}(\delta)$ as $\delta \downarrow 0$. 
Lemma 4.2.6. (i) $\lim _{\delta \downarrow 0} a_{\alpha}(\delta)=a^{*}$.

(ii) $\lim _{\delta \downarrow 0} c_{\alpha}(\delta)=0$.

Proof. (i) The family $\left(a_{\alpha}(\delta)\right)_{\delta \in I_{0}(\alpha)}$ is bounded. We show that the only possible limit of its subsequences is $a^{*}$. Assume that $a_{\delta_{n}} \rightarrow a_{\infty}$ as $n \rightarrow \infty$, with $a_{\infty} \in\left[2, a_{0}\right]$. Since $\delta \mapsto \psi_{A, B}\left(a_{\alpha}(\delta), \delta\right)$ is non-decreasing, we get

$$
\psi_{A B}\left(a_{\delta_{n}}, \delta_{n}\right)-\psi_{A B}\left(a^{*}, 0\right) \geq 0
$$

Lemma 4.2.5 tells us that the r.h.s. of (4.2.11) tends to $\psi_{A B}\left(a_{\infty}, 0\right)-\psi_{A B}\left(a^{*}, 0\right)$ as $n \rightarrow \infty$. Thus, $\psi_{A B}\left(a_{\infty}, 0\right) \geq \psi_{A B}\left(a^{*}, 0\right)$ and, since $a^{*}$ is the unique maximiser of $\psi_{A, B}(a, 0)$ (by Lemma 4.2.4), we obtain that $a_{\infty}=a^{*}$. This implies that $a_{\alpha}(\delta)$ tends to $a^{*}$ as $\delta \downarrow 0$.

(ii) The family $\left(c_{\alpha}(\delta)\right)_{\delta \in I_{0}}$ is bounded, because $c_{\alpha}(\delta) \leq a_{\alpha}(\delta)-1 \leq a_{0}-1$ for every $\delta \in I_{0}$. Assume that $c_{\alpha}\left(\delta_{n}\right) \rightarrow c_{\infty}$ as $n \rightarrow \infty$. Since $a_{\alpha}\left(\delta_{n}\right) \rightarrow a^{*}$, we necessarily have $c_{\infty} \leq a^{*}-1$. Moreover, $\left(\mu_{\alpha}\left(\delta_{n}\right)\right)_{n \in \mathbb{N}}$ is bounded above by $\mu_{0}$ (by Lemma 4.2.2). Therefore, we can pick a subsequence satisfying $\mu_{\alpha}\left(\delta_{n}\right) \rightarrow \mu_{\infty}$ as $n \rightarrow \infty$. We now recall (4.2.4) and write

$$
\begin{aligned}
\psi_{A B}\left(a_{\alpha}\left(\delta_{n}\right), \delta_{n}\right)= & \frac{1}{a_{\alpha}\left(\delta_{n}\right)} c_{\alpha}\left(\delta_{n}\right) \phi^{\mathcal{I}}\left(\mu_{\alpha}\left(\delta_{n}\right), \delta_{n}\right) \\
& +\frac{1}{a_{\alpha}\left(\delta_{n}\right)}\left[\left(a_{\delta_{n}}-c_{\alpha}\left(\delta_{n}\right)\right) \kappa\left(a_{\alpha}(\delta)-c_{\alpha}\left(\delta_{n}\right), 1-c_{\alpha}\left(\delta_{n}\right) / \mu\right)\right] .
\end{aligned}
$$

Let $n \rightarrow \infty$. Then Lemma 4.2.5 tells us that

$$
\psi_{A B}\left(a^{*}, 0\right)=\frac{1}{a^{*}}\left[c_{\infty} \phi^{\mathcal{I}}\left(\mu_{\infty}, 0\right)+\left(a^{*}-c_{\infty}\right) \kappa\left(a^{*}-c_{\infty}, 1-c_{\infty} / \mu_{\infty}\right)\right] .
$$

Therefore Lemma 4.2.4 gives that $c_{\infty}=0$ and consequently $c_{\alpha}(\delta)$ tends to 0 as $\delta \downarrow 0$.

\subsection{Proof of Proposition 4.1.1.}

Proof. Along the way we need the following. Let $\partial \phi^{\mathcal{I}} / \partial \beta^{+}$and $\partial \phi^{\mathcal{I}} / \partial \beta^{-}$denote the right- and left-derivative of $\phi^{\mathcal{I}}$, respectively.

Lemma 4.3.1. For all $\mu \geq 1$ and $\alpha, \beta \geq 0$ such that $\phi^{\mathcal{I}}(\alpha, \beta ; \mu)>\hat{\kappa}(\mu)$,

$$
\frac{\partial \phi^{\mathcal{I}}}{\partial \beta^{+}}(\alpha, \beta ; \mu) \geq \frac{\partial \phi^{\mathcal{I}}}{\partial \beta^{-}}(\alpha, \beta ; \mu)>0 .
$$

Proof. Use that $\phi^{\mathcal{I}}(\alpha, \beta ; \mu)$ is convex in $\beta$ and that $\phi^{\mathcal{I}}(\alpha, \beta ; \mu) \geq \phi^{\mathcal{I}}(\alpha, 0 ; \mu)=\hat{\kappa}(\mu)$ for all $\beta \geq 0$. 
What Lemma 4.3.1 says is that the localized phase of $\phi^{\mathcal{I}}(\alpha, \beta ; \mu)$ for fixed $\mu$ corresponds to pairs $(\alpha, \beta)$ satisfying $\phi^{\mathcal{I}}(\alpha, \beta ; \mu)>\hat{\kappa}(\mu)$.

Step 1. Recall (2.1.8) and pick a $\gamma \in(0,1)$ for which $M_{\gamma} \in \mathcal{R}(p)$. By picking $a_{A A}=a_{A B}=a^{*}=\frac{5}{2}$ and $\left(\rho_{k l}\right)=M_{\gamma}$ in (2.1.11), and noting that $\psi_{A A}\left(a^{*}\right)=$ $f\left(\alpha, \beta_{c}(\alpha) ; p\right)=\kappa\left(a^{*}, 1\right)=\varpi$, we get

$$
T_{\alpha}(\delta) \geq \gamma\left[\psi_{A B}\left(a^{*}, \delta\right)-\kappa\left(a^{*}, 1\right)\right] .
$$

Since $\mu \mapsto \phi^{\mathcal{I}}(\mu, 0)$ is continuous and $\phi^{\mathcal{I}}(1,0)=0$, Proposition 2.3.4 allows us to choose a $\mu_{\alpha} \geq 1$ that is a solution of the equation $\phi^{\mathcal{I}}(\mu, 0)=\varpi+(1 / \mu) \varsigma$ (recall (3.1.2)). Pick $C \in(0,1)$ and, in the variational formula for $\psi_{A B}\left(a^{*}, \delta\right)$ in Lemma 2.3.2, pick $c=C \delta$ and $c / b=\mu_{\alpha}$, to obtain the lower bound

$$
T_{\alpha}(\delta) \geq \frac{\gamma}{a^{*}}\left[C \delta \phi^{\mathcal{I}}\left(\mu_{\alpha}, \delta\right)+\left(a^{*}-C \delta\right) \kappa\left(a^{*}-C \delta, 1-C \delta / \mu_{\alpha}\right)-a^{*} \kappa\left(a^{*}, 1\right)\right] .
$$

Use Lemma 2.2.1(iv-vi) to Taylor expand

$$
\begin{aligned}
\kappa\left(a^{*}-C \delta, 1-C \delta / \mu_{\alpha}\right)= & \kappa\left(a^{*}, 1\right)-\left(\varsigma / a^{*}\right) C \delta / \mu_{\alpha}+B_{\alpha} C^{2} \delta^{2} \\
& +\zeta(C \delta, C \delta / \mu) C^{2} \delta^{2}\left(1+1 / \mu_{\alpha}^{2}\right), \quad \delta \downarrow 0,
\end{aligned}
$$

for some $B_{\alpha} \in \mathbb{R}$ and $\zeta$ a function on $\mathbb{R}^{2}$ tending to zero at $(0,0)$. Since $\beta_{c}(\alpha) \leq \beta^{*}$ for $\alpha \geq \alpha^{*}$, Lemma 2.4.1 tells us that $\phi^{\mathcal{I}}\left(\alpha, \beta_{c}(\alpha) ; \mu\right)$ tends to 0 as $\mu \rightarrow \infty$ uniformly in $\alpha \geq \alpha^{*}$. Consequently, $\mu_{\alpha}$ is bounded uniformly in $\alpha \geq \alpha^{*}$, and therefore so is $B_{\alpha}$. By inserting (4.3.4) into (4.3.3), we obtain that there exist $M \in \mathbb{R}$ and $\delta_{0}>0$ such that

$$
T_{\alpha}(\delta) \geq \frac{\gamma}{a^{*}}\left[C \delta\left\{\phi^{\mathcal{I}}\left(\mu_{\alpha}, \delta\right)-\phi^{\mathcal{I}}\left(\mu_{\alpha}, 0\right)\right\}+M a^{*} C^{2} \delta^{2}\right] \quad \forall \alpha>\alpha^{*}, \delta \in I_{0}(\alpha) .
$$

Since, by Lemma 2.2.2(iv) and Proposition 2.3.4, $\phi^{\mathcal{I}}\left(\mu_{\alpha}, 0\right)>\hat{\kappa}\left(\mu_{\alpha}\right)$, Lemma 4.3.1 gives that $\left(\alpha, \beta_{c}(\alpha)\right)$ lies in the localized phase of $\left(\alpha^{\prime}, \beta^{\prime}\right) \rightarrow \phi^{\mathcal{I}}\left(\mu_{\alpha}, \alpha^{\prime}, \beta^{\prime}\right)$. Therefore

$$
\phi^{\mathcal{I}}\left(\mu_{\alpha}, \delta\right)-\phi^{\mathcal{I}}\left(\mu_{\alpha}, 0\right) \geq C_{\alpha}^{\prime} \delta \quad \text { with } \quad C_{\alpha}^{\prime}=\frac{\partial \phi^{\mathcal{I}}}{\partial \beta^{+}}\left(\alpha, \beta_{c}(\alpha) ; \mu_{\alpha}\right) \in(0,1] .
$$

Hence (4.3.5) becomes

$$
T_{\alpha}(\delta) \geq \frac{\gamma}{a^{*}}\left(C C_{\alpha}^{\prime}+M a^{*} C^{2}\right) \delta^{2} \quad \forall \alpha>\alpha^{*}, \delta \in I_{0}(\alpha) .
$$

Now pick $C$ small enough so that $M a^{*} C>-\frac{1}{2} C_{\alpha}^{\prime}$, to get the inequality in (4.1.3) with $C_{1}=\frac{\gamma}{2 a^{*}} C C_{\alpha}^{\prime}$.

Step 2. To complete the proof of Proposition 4.1.1 it suffices to show that $C_{\alpha}^{\prime}$ can be bounded from below by a strictly positive constant. The latter is done as follows. Suppose that there exists a sequence $\left(\alpha_{n}\right)_{n \in \mathbb{N}}$ in $\left(\alpha^{*}, \infty\right]$ such that $\lim _{n \rightarrow \infty} C_{\alpha_{n}}^{\prime}=0$. By considering a subsequence of $\left(\alpha_{n}\right)_{n \in \mathbb{N}}$, we may assume that $\alpha_{n}$ and $\mu_{\alpha_{n}}$ converge, respectively, to $\alpha_{\infty} \in\left[\alpha^{*}, \infty\right]$ and $\mu_{\infty}$. Moreover, as proved in Lemma 4.2.5,

$$
\lim _{n \rightarrow \infty} \phi^{\mathcal{I}}\left(\alpha_{n}, \beta, \mu_{\alpha_{n}}\right)=\phi^{\mathcal{I}}\left(\alpha_{\infty}, \beta, \mu_{\infty}\right) \quad \forall \beta>0,
$$


and $\beta \mapsto \phi^{\mathcal{I}}\left(\alpha_{n}, \beta ; \mu_{\alpha_{n}}\right)$ is convex for every $n \in \mathbb{N}$. Consequently,

$$
\frac{\partial \phi^{\mathcal{I}}}{\partial \beta^{-}}\left(\alpha_{\infty}, \beta_{c}\left(\alpha_{\infty}\right) ; \mu_{\infty}\right) \leq \limsup _{n \rightarrow \infty} \frac{\partial \phi^{\mathcal{I}}}{\partial \beta^{+}}\left(\alpha_{n}, \beta_{c}\left(\alpha_{n}\right) ; \mu_{\alpha_{n}}\right)=\limsup _{n \rightarrow \infty} C_{\alpha_{n}}^{\prime}=0
$$

and

$$
\phi^{\mathcal{I}}\left(\alpha_{\infty}, \beta ; \mu_{\infty}\right)=\varpi+\frac{1}{\mu_{\infty}} \varsigma>\hat{\kappa}\left(\mu_{\infty}\right)
$$

But (4.3.9) yields $\frac{\partial \phi^{\mathcal{I}}}{\partial \beta^{-}}\left(\alpha_{\infty}, \beta_{c}\left(\alpha_{\infty}\right) ; \mu_{\infty}\right) \leq 0$, which contradicts the statement in Lemma 4.2.5, because of (4.3.10).

4.4. Proof of Proposition 4.1.2. Step 1. Since $\psi_{A B} \geq \psi_{k l}$ for all $k l \in\{A, B\}^{2}$, we can write

$$
f\left(\alpha, \beta_{c}(\alpha)+\delta ; p\right)-f\left(\alpha, \beta_{c}(\alpha) ; p\right) \leq \psi_{A B}\left(a_{\alpha}(\delta), \delta\right)-\varpi .
$$

Because of Lemma 4.2.4 we also have

$$
f\left(\alpha, \beta_{c}(\alpha)+\delta ; p\right)-f\left(\alpha, \beta_{c}(\alpha) ; p\right) \leq \psi_{A B}\left(a_{\alpha}(\delta), \delta\right)-\psi_{A B}\left(a_{\alpha}(\delta), 0\right) .
$$

Since

$$
\begin{aligned}
& \psi_{A B}\left(a_{\alpha}(\delta), \delta\right)-\psi_{A B}\left(a_{\alpha}(\delta), 0\right) \\
& \leq \frac{1}{a_{\alpha}(\delta)}\left\{c_{\alpha}(\delta)\left[\phi^{\mathcal{I}}\left(\mu_{\alpha}(\delta), \alpha, \beta_{c}(\alpha)+\delta\right)-\phi^{\mathcal{I}}\left(\mu_{\alpha}(\delta), \alpha, \beta_{c}(\alpha)\right)\right]\right\}
\end{aligned}
$$

and, for $\delta$ fixed, $\beta \mapsto \phi^{\mathcal{I}}\left(\alpha, \beta ; \mu_{\alpha}(\delta)\right)$ is convex with slope bounded by 1 , we obtain

$$
\begin{aligned}
\psi_{A B}\left(a_{\alpha}(\delta), \delta\right)-\psi_{A B}\left(a_{\alpha}(\delta), 0\right) & \leq \frac{1}{a_{0}}\left[\left(\frac{\partial}{\partial \beta} \phi^{\mathcal{I}}\right)\left(\alpha, \beta_{c}(\alpha)+\delta ; \mu_{\alpha}(\delta)\right)\right] c_{\alpha}(\delta) \delta \\
& \leq \frac{1}{a_{0}} c_{\alpha}(\delta) \delta
\end{aligned}
$$

Step 2. The proof of (4.1.4) is now completed by the following.

Lemma 4.4.1. For every $\alpha>\alpha^{*}$ there exist $C_{\alpha}<\infty$ and $\delta_{0}>0$ such that $c_{\alpha}(\delta) \leq C_{\alpha} \delta$ for all $\delta \in I_{0}(\alpha)$.

Proof. Recall the statement of Lemma 4.2.2, i.e., for every $\delta \in I_{0}(\alpha)$ there exists a $\mu_{\alpha}(\delta) \in\left[1, \mu_{0}\right]$ such that

$$
\psi_{A B}\left(a_{\alpha}(\delta), \delta\right)=\sup _{c \leq \min \left\{a_{\alpha}(\delta)-1, \mu_{\alpha}(\delta)\left(a_{\alpha}(\delta)-2\right) /\left(\mu_{\alpha}(\delta)-1\right)\right\}} H\left(c, a_{\alpha}(\delta), \mu_{\alpha}(\delta), \delta\right)
$$

with

$$
H\left(c, a_{\alpha}(\delta), \mu_{\alpha}(\delta), \delta\right)=\frac{1}{a_{\alpha}(\delta)}\left[c \phi^{\mathcal{I}}\left(\mu_{\alpha}(\delta), \delta\right)+\left(a_{\alpha}(\delta)-c\right) \kappa\left(a_{\alpha}(\delta)-c, 1-c / \mu_{\alpha}(\delta)\right)\right] .
$$


We proved in Lemma 4.2.6 that the supremum is attained in a point $c_{\alpha}(\delta)>0$ that tends to zero as $\delta \downarrow 0$. Since $H$ is differentiable w.r.t. its first variable, we have

$$
\frac{\partial H}{\partial 1}\left(c_{\alpha}(\delta), a_{\alpha}(\delta), \mu_{\alpha}(\delta), \delta\right)=0 .
$$

Moreover, since $H$ is also differentiable w.r.t. its second variable, and since the maximum of $\psi_{A B}(a, \delta)$ over $a \in[2, \infty)$ is attained in $a_{\alpha}(\delta)$, we have

$$
\frac{\partial H}{\partial 2}\left(c_{\alpha}(\delta), a_{\alpha}(\delta), \mu_{\alpha}(\delta), \delta\right)=0 .
$$

In what follows, we consider three functions $\left(\delta \mapsto \xi_{i, \alpha}(\delta)\right)_{i=1,2,3}$ that tend to zero as $\delta \downarrow 0$. Since $a_{\alpha}(\delta)$ tends to $a^{*}$ by Lemma 4.2.6(i), we use the notation $a_{\alpha}(\delta)=a^{*}+\hat{a}_{\alpha}(\delta)$. For simplicity, when we do not indicate the point at which a derivative is taken, this point is $\left(a^{*}, 1\right)$ by default.

Computing the derivative in (4.4.7) from (4.4.6), we obtain a relation between $c_{\alpha}(\delta)$ and $a_{\alpha}(\delta)$. We may simplify this relation by using a first order Taylor expansion of the quantities

$$
\begin{aligned}
& \kappa\left(a_{\alpha}(\delta), 1-c_{\alpha}(\delta) / \mu_{\alpha}(\delta)\right), \quad \frac{\partial \kappa}{\partial 2}\left(a_{\alpha}(\delta), 1-c_{\alpha}(\delta) / \mu_{\alpha}(\delta)\right), \\
& \frac{\partial \kappa}{\partial 2}\left(a_{\alpha}(\delta), 1-c_{\alpha}(\delta) / \mu_{\alpha}(\delta)\right),
\end{aligned}
$$

in the neighbourhood of $\left(a^{*}, 1\right)$. This gives, after some straightforward but tedious computations,

$$
\begin{aligned}
& {\left[\phi^{\mathcal{I}}\left(\mu_{\alpha}(\delta), \delta\right)-\kappa\left(a^{*}, 1\right)-\frac{5}{2 \mu_{\alpha}(\delta)} \frac{\partial K}{\partial 2}\right]} \\
& \quad+c_{\alpha}(\delta) A_{\alpha, \delta}+\hat{a}_{\alpha}(\delta) B_{\alpha, \delta}+\xi_{1, \alpha}(\delta)\left(\left|c_{\alpha}(\delta)\right|+\left|\hat{a}_{\alpha}(\delta)\right|\right)=0
\end{aligned}
$$

with

$$
\begin{aligned}
& A_{\alpha, \delta}=\frac{1}{\mu_{\alpha}(\delta)}\left[2 \frac{\partial \kappa}{\partial 2}+5 \frac{\partial^{2} \kappa}{\partial 1 \partial 2}+\frac{5}{2 \mu_{\alpha}(\delta)} \frac{\partial^{2} \kappa}{\partial 2^{2}}+\frac{5 \mu_{\alpha}(\delta)}{2} \frac{\partial^{2} \kappa}{\partial 2^{2}}\right], \\
& B_{\alpha, \delta}=-\frac{1}{\mu_{\alpha}(\delta)}\left[\frac{\partial \kappa}{\partial 2}+\frac{5}{2} \frac{\partial^{2} \kappa}{\partial 1 \partial 2}+\frac{5 \mu_{\alpha}(\delta)}{2} \frac{\partial^{2} \kappa}{\partial 1^{2}}\right] .
\end{aligned}
$$

The same type of computation applied to (4.4.8) gives

$$
\hat{a}_{\alpha}(\delta)+\xi_{2, \alpha}(\delta) \hat{a}_{\alpha}(\delta)=c_{\alpha}(\delta) C_{\alpha, \delta}+\xi_{3, \alpha}(\delta) c_{\alpha}(\delta)
$$

with

$$
C_{\alpha, \delta}=-\left(\frac{2}{5}\right)^{2} \frac{\kappa\left(a^{*}, 1\right)-\phi^{\mathcal{I}}\left(\mu_{\alpha}(\delta), \delta\right)}{\frac{\partial^{2} \kappa}{\partial 1^{2}}}+1+\frac{\frac{\partial \kappa^{2}}{\partial 1 \partial 2}}{\mu_{\alpha}(\delta) \frac{\partial^{2} \kappa}{\partial 1^{2}}} .
$$

Recalling that $c_{\alpha}(\delta)$ and $\hat{a}_{\alpha}(\delta)$ tend to zero as $\delta \downarrow 0$ (by Lemma 4.2.6), we obtain from (4.4.12) that $\hat{a}_{\alpha}(\delta) \in\left[\left(C_{\alpha, \delta}-\varepsilon\right) c_{\alpha}(\delta),\left(C_{\alpha, \delta}+\varepsilon\right) c_{\alpha}(\delta)\right]$ for all $\varepsilon>0$ and $\delta$ small enough. From this last inclusion and (4.4.10), we get that there exists a $\delta_{1}>0$ such that, for all $\varepsilon>0$ and $\delta \leq \delta_{1}$,

$$
\left[\phi^{\mathcal{I}}\left(\mu_{\alpha}(\delta), \delta\right)-\kappa\left(a^{*}, 1\right)-\frac{5}{2 \mu_{\alpha}(\delta)} \frac{\partial K}{\partial 2}\right]+c_{\alpha}(\delta)\left(A_{\alpha, \delta}+B_{\alpha, \delta} C_{\alpha, \delta}+\varepsilon\right) \geq 0 .
$$


Abbreviate

$$
\Delta(\delta)=\phi^{\mathcal{I}}\left(\mu_{\alpha}(\delta), \delta\right)-\kappa\left(a^{*}, 1\right)-\frac{5}{2 \mu_{\alpha}(\delta)} \frac{\partial K}{\partial 2} .
$$

Since $\left(\alpha, \beta_{c}(\alpha)\right)$ lies in the delocalized region, Proposition 2.3 .4 tells us that $\phi^{\mathcal{I}}\left(\mu_{\alpha}(\delta), 0\right) \leq \kappa\left(a^{*}, 1\right)+\frac{5}{2 \mu_{\alpha}(\delta)} \frac{\partial K}{\partial 2}$. Therefore we can write

$$
\Delta(\delta) \leq \phi^{\mathcal{I}}\left(\mu_{\alpha}(\delta), \delta\right)-\phi^{\mathcal{I}}\left(\mu_{\alpha}(\delta), 0\right)
$$

A simple computation gives that $\phi^{\mathcal{I}}(\mu, \delta)-\phi^{\mathcal{I}}(\mu, 0) \leq \delta$ for all $\mu \geq 1$ (recall (4.1.1)). Hence $\Delta(\delta) \leq \delta$.

From (4.4.11) and (4.4.13), we have

$$
A_{\alpha, \delta}+B_{\alpha, \delta} C_{\alpha, \delta}=\frac{A}{\mu_{\alpha}(\delta)^{2}}+\Delta(\delta)\left[\frac{B}{\mu_{\alpha}(\delta)}-\frac{2}{5}\right]
$$

with

$$
\begin{aligned}
& A=\frac{1}{\frac{\partial^{2} \kappa}{\partial 1^{2}}}\left[\frac{5}{2} \frac{\partial^{2} \kappa}{\partial 2^{2}}-\frac{2}{5}\left(\frac{\partial \kappa}{\partial 2}\right)^{2}-2 \frac{\partial \kappa}{\partial 2} \frac{\partial^{2} \kappa}{\partial 1 \partial 2}-\frac{5}{2}\left(\frac{\partial^{2} \kappa}{\partial 1 \partial 2}\right)^{2}\right] \text { and } \\
& B=\frac{1}{\frac{\partial^{2} \kappa}{\partial 1^{2}}}\left[-\left(\frac{2}{5}\right)^{2} \frac{\partial \kappa}{\partial 2}-\frac{2}{5} \frac{\partial^{2} \kappa}{\partial 1 \partial 2}\right] .
\end{aligned}
$$

By inserting the values of the derivatives given in Lemma 2.2.1(v-vi), we find that $A<0$. Thus, recalling that $1 \leq \mu_{\alpha}(\delta) \leq \mu_{0}$ for all $\delta \in I_{0}(\alpha)$ (by Lemma 4.2.2), we can rewrite (4.4.14) as

$$
A_{\alpha, \delta}+B_{\alpha, \delta} C_{\alpha, \delta} \leq \frac{A}{\mu_{0}^{2}}+\Delta(\delta)\left[|B|+\frac{2}{5}\right]
$$

Since $\Delta(\delta) \leq \delta$, we can now assert that there exists a $\delta_{2}>0$ such that $0<\delta \leq \delta_{2}$ implies $A_{\alpha, \delta}+B_{\alpha, \delta} C_{\alpha, \delta} \leq 3 A / 2 \mu_{0}^{2}$. Therefore (4.4.14) becomes $\delta+c_{\alpha}(\delta) 3 A / 2 \mu_{0}^{2} \geq 0$ and, consequently, for $\delta_{0}=\min \left\{\delta_{1}, \delta_{2}\right\}$ there exists a $C_{\alpha}>0$ such that for all $\delta \in I_{0}(\alpha)$,

$$
c_{\alpha}(\delta) \leq C_{\alpha} \delta
$$

This completes the proof of Lemma 4.4.1.

\section{Proof of Theorem 1.4.3}

In Sect. 5.1 we study a variation of the single linear interface model in which the variable $\mu$ is replaced by a dual variable $\lambda$, which enters into the Hamiltonian rather than in the set of paths. We show that the free energy for this dual model is smooth. In Sect. 5.2 we show that the dual free energy has a non-zero curvature. In Sects. 5.3 and 5.4 we use this to prove that $\phi^{\mathcal{I}}$ and $\psi_{A B}$ are smooth on their localized phases and have a non-zero curvature too. The latter in turn are used in Sect. 5.5 to prove the smoothness of $f$ on $\mathcal{L}$. Key ingredients in the proofs are the implicit function theorem, the exponential tightness of the excursions in the localized phases, and the uniqueness of the maximisers in the variational formulas for $\phi^{\mathcal{I}}, \psi_{A B}$ and $f$. 
5.1. Fenchel-Legendre transform of $\phi^{\mathcal{I}}$. We begin by defining the dual of the single interface model. Let $\mathcal{W}_{L}$ be the set of $L$-step directed self-avoiding paths that start at $(0,0)$ and end at $(x, 0)$ for some $x \in\{1, \ldots, L\}$. For $\pi \in \mathcal{W}_{L}$, let $h(\pi)$ be the number of horizontal steps in $\pi$. For $\lambda \geq 0$, define (recall (2.4.2))

$$
\begin{aligned}
U_{L}^{\omega, \mathcal{I}}(\alpha, \beta ; \lambda) & =\sum_{\pi \in \mathcal{W}_{L}} e^{-\lambda h(\pi)-H_{L}^{\omega, \mathcal{I}}(\pi)} \\
u^{\mathcal{I}}(\alpha, \beta ; \lambda) & =\lim _{L \rightarrow \infty} \frac{1}{L} \log U_{L}^{\omega, \mathcal{I}}(\alpha, \beta ; \lambda) \omega-\text { a.s. }
\end{aligned}
$$

and

$$
\tilde{\kappa}(\lambda)=\lim _{L \rightarrow \infty} \frac{1}{L} \log \sum_{\pi \in \mathcal{W}_{L}} e^{-\lambda h(\pi)} .
$$

The convergence $\omega$-a.s. and in mean and the constantness $\omega$-a.s. of $u^{\mathcal{I}}(\alpha, \beta ; \lambda)$ follow from the subadditive ergodic theorem (Kingman [8]). Set

$$
\mathcal{L}_{u}=\left\{(\alpha, \beta, \lambda)=\operatorname{CONE} \times[0, \infty): u^{\mathcal{I}}(\alpha, \beta ; \lambda)>\tilde{\kappa}(\lambda)\right\},
$$

i.e., the region where the dual of the single linear interface model is localized.

Proposition 5.1.1. The function $(\alpha, \beta, \lambda) \mapsto u^{\mathcal{I}}(\alpha, \beta ; \lambda)$ is infinitely differentiable on $\mathcal{L}_{u}$.

Proof. The proof is similar to that of the infinite differentiability of the free energy for the single interface model, proved in Giacomin and Toninelli [6]. Therefore, we only sketch the main steps in the proof and refer to [6] for further details.

Step 1. The claim follows from the Arzela-Ascoli theorem as soon as we prove that for all $\left(\alpha_{0}, \beta_{0}, \lambda_{0}\right) \in \mathcal{L}_{u}$ there exists $\mathcal{V} \subset \mathcal{L}_{u}$ a neighborhood of $\left(\alpha_{0}, \beta_{0}, \lambda_{0}\right)$ such that for all $k \in \mathbb{N}$, the $k_{t h}$ derivative of $L^{-1} \mathbb{E}\left(\log U_{L}^{\omega, \mathcal{I}}(\alpha, \beta ; \lambda)\right)$ w.r.t. any of the parameters $\alpha, \beta, \lambda$ is bounded uniformly in $L$ and $(\alpha, \beta, \lambda) \in \mathcal{V}$, where $\mathbb{E}$ denotes expectation w.r.t. $\omega$.

For $a, b \in \mathbb{N}$ with $a<b$, let $\mathcal{H}_{a, b}$ be the set of bounded functions that are measurable w.r.t. the $\sigma$-algebra $\sigma\left(\pi_{j}: j \in\{a, \ldots, b\}\right)$. As explained in [6], the conditions of the Arzela-Ascoli theorem are satisfied once we show that for all $\left(\alpha_{0}, \beta_{0}, \lambda_{0}\right) \in \mathcal{L}_{u}$ there exist $C_{1}, C_{2}>0$ and $\mathcal{V} \subset \mathcal{L}_{u}$ such that, for all $a_{1}, b_{1}, a_{2}, b_{2} \in \mathbb{N}$ with $a_{1}<b_{1}<a_{2}<$ $b_{2} \leq L$ and $\left(f_{1}, f_{2}\right) \in \mathcal{H}_{a_{1}, b_{1}} \times \mathcal{H}_{a_{2}, b_{2}}$ and $(\alpha, \beta, \lambda) \in \mathcal{V}$, the following inequality holds:

$$
\mathbb{E}\left(E_{L}^{\omega, \mathcal{I}}\left(f_{1} f_{2}\right)-E_{L}^{\omega, \mathcal{I}}\left(f_{1}\right) E_{L}^{\omega, \mathcal{I}}\left(f_{2}\right)\right) \leq C_{1}\left\|f_{1}\right\|_{\infty}\left\|f_{2}\right\|_{\infty} e^{-C_{2}\left(a_{2}-b_{1}\right)} .
$$

Here, $E_{L}^{\omega, \mathcal{I}}$ is expectation w.r.t. the law of the $L$-step copolymer at fixed $\omega$ given by (recall (5.1.1))

$$
P_{L}^{\omega, \mathcal{I}}(\pi)=\frac{1}{U_{L}^{\omega, \mathcal{I}}} e^{-\lambda h(\pi)-H_{L}^{\omega, \mathcal{I}}(\pi)} .
$$


Next, the correlation inequality in (5.1.4) will follow once we show that there exist $C_{1}, C_{2}>0$ and $\mathcal{V} \subset \mathcal{L}_{u}$ (depending on $\left.\alpha_{0}, \beta_{0}, \lambda_{0}\right)$ such that, for all $a, b, L \in \mathbb{N}$ with $a \leq b \leq L$, we have

$$
\mathbb{E}\left(\left[P_{L}^{\omega, \mathcal{I}}\right]^{\otimes 2}\left(B_{a, b}\right)\right) \leq C_{1} e^{-C_{2}(b-a)},
$$

where $\left[P_{L}^{\omega, \mathcal{I}}\right]^{\otimes 2}$ is the joint law of two independent copies of the $L$-step copolymer at fixed $\omega$, and

$$
\begin{aligned}
& B_{a, b}=\left\{\left(\pi^{1}, \pi^{2}\right): \nexists j \in\{a, \ldots, b\} \text { such that the } j_{t h}\right. \text { steps } \\
& \left.\quad \text { of } \pi_{1} \text { and } \pi_{2} \text { are the same and occur at the same height }\right\} .
\end{aligned}
$$

Indeed, on $\left[B_{a, b}\right]^{c}$ the two paths can be coupled as soon as they make the common step. An example of a pair of paths $\left(\pi_{1}, \pi_{2}\right)$ not in $B_{a, b}$ is displayed in Fig. 10.

Step 2. For $i=1,2$ and $M \in \mathbb{N}$, let $l_{i, M}$ be the number of excursions of $\pi_{i}$ (either strictly positive or non-positive) that are included in $\{a, \ldots, b\}$ and are smaller than or equal to $M$. Let

$$
\mathcal{E}_{M}\left(\pi_{i}\right)=\left\{\left(b_{1}^{i}, e_{1}^{i}\right), \ldots,\left(b_{l_{i, M}}^{i}, e_{l_{i, M}}^{i}\right)\right\}
$$

where $\left(b_{j}^{i}, e_{j}^{i}\right)$ denote the end-steps of the $j_{t h}$ excursion. Put $\tau_{j}^{i}=e_{j}^{i}-b_{j}^{i}+1$, and for $\gamma \in(0,1)$ let

$$
\mathcal{A}_{i, \gamma, M}=\left\{\pi_{i}: \sum_{j=1}^{l_{i, M}} \tau_{j}^{i} \geq \gamma(b-a)\right\} .
$$

Lemma 5.1.2. (i) For all $\gamma_{0} \in(0,1)$ and $\left(\alpha_{0}, \beta_{0}, \lambda_{0}\right) \in \mathcal{L}_{u}$ there exist $M \in \mathbb{N}$, an open neighborhood $\mathcal{V}$ of $\left(\alpha_{0}, \beta_{0}, \lambda_{0}\right)$ in $\mathcal{L}_{u}$ and $C_{1}, C_{2}>0$ such that, for $L \geq b \geq a$ and $(\alpha, \beta, \lambda) \in \mathcal{V}$,

$$
\mathbb{E}\left(\mathbb{P}_{L}^{\omega, \mathcal{I}}\left(\mathcal{A}_{i, \gamma_{0}, M}\right)\right) \geq 1-C_{1} e^{-C_{2}(b-a)}, \quad i=1,2 .
$$

(ii) For all $T_{0} \in \mathbb{N}$ and $\left(\alpha_{0}, \beta_{0}, \lambda_{0}\right) \in \mathcal{L}_{u}$ there exist $\gamma \in(0,1)$, an open neighborhood $\mathcal{V}$ of $\left(\alpha_{0}, \beta_{0}, \lambda_{0}\right)$ in $\mathcal{L}_{u}$ and $C_{1}, C_{2}>0$ such that, for all $L \geq b \geq a$ and $(\alpha, \beta, \lambda) \in \mathcal{V}$,

$$
\mathbb{E}\left(\mathbb{P}_{L}^{\omega, \mathcal{I}}\left(\mathcal{A}_{i, \gamma, T_{0}}\right)\right) \leq C_{1} e^{-C_{2}(b-a)}, \quad i=1,2
$$

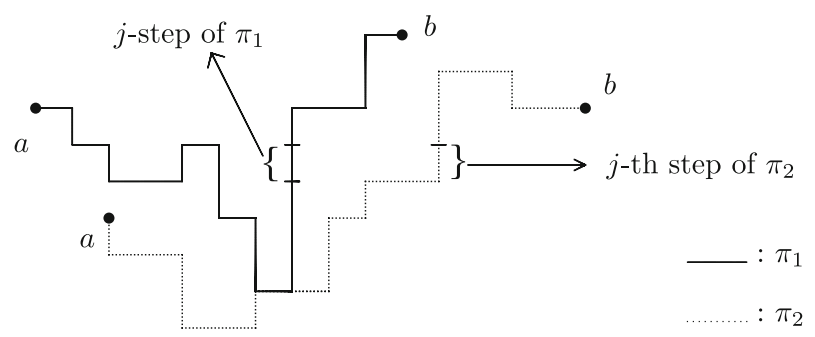

Fig. 10. A pair of paths $\left(\pi_{1}, \pi_{2}\right)$ whose $j_{t h}$ steps are the same and occur at the same height 
Proof. (i) This part gives the exponential tightness of the excursions of the copolymer in the localized phase. Compared to Proposition 3.1.1, both the model and the statement are different. However, the same tools can be used and for this reason we only give a sketch of the proof. By the definition of $\mathcal{A}_{i, \gamma_{0}, M}^{c}$, there are two cases. Case 1 . The sum of the lengths of the strictly positive excursions larger than $M$ in $\{a, \ldots, b\}$ is $\geq \gamma \frac{b-a}{2}$.

Case 2. The sum of the lengths of the non-positive excursions larger than $M$ in $\{a, \ldots, b\}$ is $\geq \gamma \frac{b-a}{2}$.

In Case 1 , by concatenating the strictly positive excursions larger than $M$ in $\{a, \ldots, b\}$, we can bound the total entropy carried by these excursions (i.e., the logarithm of their total cardinality) from above by the entropy of a large single positive excursion whose length is equal to the sum of the lengths of the excursions larger than $M$, which is at least $\gamma \frac{b-a}{2}$. This provides an upper bound for the analogue of the sum in (3.1.14). Next, the gain in the free energy obtained by replacing the large single positive excursion by a path with the same endpoints but no positivity constraint is, for $b-a$ large enough, of order $\exp \left[C_{2}(b-a)\right]$, with $C_{2}=\frac{\gamma}{2}[u(\lambda)-\tilde{\kappa}(\lambda)]$. This provides a lower bound for the normalizing partition sum in (3.1.14). By choosing a small enough open neighborhood $\mathcal{V}$ of $\left(\alpha_{0}, \beta_{0}, \lambda_{0}\right)$ in $\mathcal{L}_{u}$, we get that there exists a $c>0$ such that, for all $(\alpha, \beta, \lambda) \in \mathcal{V}$, we have $u(\alpha, \beta ; \lambda)-\tilde{\kappa}(\lambda) \geq c$. Thus, $\frac{c \gamma}{2}$ is a lower bound for $C_{2}$, uniform in $\mathcal{V}$. In Case 2, a similar argument applies.

(ii) Again we only sketch the proof. We partition $\{a, \ldots, b\}$ into $\frac{b-a}{R}$ blocks of size $R$. A block is called "good" if it carries only monomers of type $A$. By the law of large numbers, there exists a $c_{R}>0$ such that approximately $c_{R}(b-a)$ of the blocks are good. We can therefore choose $\gamma$ close enough to 1 such that, on $\mathcal{A}_{1, \gamma, T}$, at least $\frac{c_{R}}{2}(b-a)$ of the good blocks are covered only by excursions smaller than $T$. Such blocks are called "good $T$-blocks". Consequently, more than $\frac{R}{T}$ excursions are required to cover a good $T$-block and so at least $\frac{R}{T}$ steps in each good $T$-block are below the interface. Thus, after relaxing the condition $\mathcal{A}_{1, \gamma, T}$ in the normalizing partition sum, we can replace on each good $T$-block the excursions smaller than $T$ by a large strictly positive excursion. This does not decrease the entropy, but increases the energy by at least $\beta \frac{R}{T}$ on each good $T$-block. Summed up these energy increases are of order $\frac{c_{R}}{2}(b-a) \beta \frac{R}{T}$.

Step 3. Let $D=\mathcal{A}_{1, \frac{3}{4}, M} \cap \mathcal{A}_{2, \frac{3}{4}, M}$ and $\mathcal{T}_{M}=\left\{\mathcal{E}_{M}\left(\pi_{1}\right): \pi_{1} \in \mathcal{A}_{1, \frac{3}{4}, M}\right\}$. For $i=1,2$ and $\mathcal{E}_{M} \in \mathcal{T}_{M}$, let $\mathcal{J}^{i}\left(\mathcal{E}_{M}\right)=\left\{\pi_{i}: \mathcal{E}_{M}\left(\pi_{i}\right)=\mathcal{E}_{M}\right\}$. Then Lemma 5.1.2 applied at $\gamma_{0}=\frac{3}{4}$ implies that there exists $M \in \mathbb{N}$, an open neighborhood $\mathcal{V}$ of $\left(\alpha_{0}, \beta_{0}, \lambda_{0}\right)$ in $\mathcal{L}_{u}$ and $C_{1}, C_{2}>0$ such that for $L \geq b$ and $(\alpha, \beta, \lambda) \in \mathcal{V}$ we have $\left[P_{L}^{\omega, \mathcal{I}}\right]^{\otimes 2}\left(D^{c}\right) \leq$ $2 C_{1} e^{-C_{2}(b-a)}$, so that it remains to estimate $\left[P_{L}^{\omega, \mathcal{I}}\right]^{\otimes 2}\left(B_{a, b} \cap D\right)$,

$$
\begin{aligned}
& {\left[P_{L}^{\omega, \mathcal{I}}\right]^{\otimes 2}\left(B_{a, b} \cap D\right)} \\
& =\sum_{\mathcal{E}_{M}^{1}, \mathcal{E}_{M}^{2} \in \mathcal{T}_{M}}\left[P_{L}^{\omega, \mathcal{I}}\right]^{\otimes 2}\left(B_{a, b} \cap\left\{\mathcal{J}^{1}\left(\mathcal{E}_{M}^{1}\right) \times \mathcal{J}^{2}\left(\mathcal{E}_{M}^{2}\right)\right\}\right) \\
& =\sum_{\mathcal{E}_{M}^{1}, \mathcal{E}_{M}^{2} \in \mathcal{T}_{M}} E_{L}^{\omega, \mathcal{I}}\left(1_{\left\{\pi_{2} \in \mathcal{J}^{2}\left(\mathcal{E}_{M}^{2}\right)\right\}} P_{L}^{\omega, \mathcal{I}}\left(B_{a, b} \cap\left\{\pi_{1} \in \mathcal{J}^{1}\left(\mathcal{E}_{M}^{1}\right)\right\} \mid \pi_{2}\right)\right) .
\end{aligned}
$$


Next, set $\tilde{i}=2$ if $i=1$ and vice versa, and define $\mathcal{R}^{i}\left(\mathcal{E}_{M}^{1}, \mathcal{E}_{M}^{2}\right)=\left\{j \in\left\{1, \ldots, l_{i, M}\right\}: b_{k}^{\tilde{i}}\right.$ or $e_{k}^{\tilde{i}} \in\left\{b_{j}^{i}, e_{j}^{i}\right\}$ for some $\left.k \in\left\{1, \ldots, l_{\tilde{i}, M}\right\}\right\}$.

By the definition of $\mathcal{A}_{i, \frac{3}{4}, M}$ in (5.1.9), for any $\mathcal{E}_{M}^{1}, \mathcal{E}_{M}^{2} \in \mathcal{T}_{M}$ there are at least $\frac{1}{4}(b-a)$ steps in $\{a, \ldots, b\}$ belonging to excursions smaller than $M$, in both $\pi_{1}$ and $\pi_{2}$. Therefore we can choose a $C>0$ small enough such that, for all $\mathcal{E}_{M}^{1}, \mathcal{E}_{M}^{2} \in \mathcal{T}_{M}$, either $\left|\mathcal{R}^{i}\left(\mathcal{E}_{M}^{1}, \mathcal{E}_{M}^{2}\right)\right| \geq C(b-a) / M$ or $\left|\mathcal{R}^{\tilde{i}}\left(\mathcal{E}_{M}^{1}, \mathcal{E}_{M}^{2}\right)\right| \geq C(b-a) / M$. Without loss of generality, we may assume that $\left|\mathcal{R}^{1}\left(\mathcal{E}_{M}^{1}, \mathcal{E}_{M}^{2}\right)\right| \geq C(b-a) / M$. Because of the condition imposed by $B_{a, b}$, for all $j \in \mathcal{R}^{1}\left(\mathcal{E}_{M}^{1}, \mathcal{E}_{M}^{2}\right)$ the excursion of $\pi_{1}$ on $\left\{b_{j}^{1}, \ldots, e_{j}^{1}\right\}$ has some prohibited parts. Indeed, $\pi_{2}$ starts or ends an excursion inside $\left\{b_{j}^{1}, \ldots, e_{j}^{1}\right\}$, which restricts the possible excursions of $\pi_{1}$, because $\pi_{1}$ cannot make the same step as $\pi_{2}$ at the same height. Moreover, there is only a finite number of possibilities to make an excursion smaller than $M$ and so, for all $j \in \mathcal{R}^{1}\left(\mathcal{E}_{M}^{1}, \mathcal{E}_{M}^{2}\right)$, relaxing the condition $B_{a, b}$ on $\left\{b_{j}^{1}, \ldots, e_{j}^{1}\right\}$ amounts to increasing the probability in (5.1.10) by a factor $Q>1$ depending only on $M$, i.e.,

$$
P_{L}^{\omega, \mathcal{I}}\left(B_{a, b} \cap\left\{\pi_{1} \in \mathcal{J}^{1}\left(\mathcal{E}_{M}^{1}\right)\right\} \mid \pi_{2}\right) \leq Q^{-\left|\mathcal{R}^{1}\left(\mathcal{E}_{M}^{1}, \mathcal{E}_{M}^{2}\right)\right|} P_{L}^{\omega, \mathcal{I}}\left(\left\{\pi_{1} \in \mathcal{J}^{1}\left(\mathcal{E}_{M}^{1}\right)\right\}\right) .
$$

Therefore, since $\left|\mathcal{R}^{1}\left(\mathcal{E}_{M}^{1}, \mathcal{E}_{M}^{2}\right)\right| \geq C(b-a) / M,(5.1 .10)$ becomes

$$
\left[P^{\omega, \mathcal{I}}\right]^{\otimes 2}\left(B_{a, b} \cap D\right) \leq e^{-C \frac{b-a}{M} \log Q},
$$

which proves (5.1.6) and completes the proof of Proposition 5.1.1.

The following proposition provides the link between $u^{\mathcal{I}}$ and $\phi^{\mathcal{I}}$.

Proposition 5.1.3. For $\lambda \geq 0$,

$$
u^{\mathcal{I}}(\lambda)=\sup _{\rho \in(0,1]}\left\{-\lambda \rho+\phi^{\mathcal{I}}(1 / \rho)\right\}
$$

Proof. For $\rho \in(0,1]$, let $\mathcal{W}_{L}(\rho)=\left\{\pi \in \mathcal{W}_{L}: h(\pi)=\rho L\right\}$ and

$$
U_{L}^{\omega, \mathcal{I}}(\lambda, \rho)=\sum_{\pi \in \mathcal{W}_{L}(\rho)} e^{-\lambda h(\pi)-H_{L}^{\omega, \mathcal{I}}(\pi)} .
$$

By restricting the sum defining $U_{L}^{\omega, \mathcal{I}}(\lambda)$ in (5.1.1) to the set $\mathcal{W}_{L}(\rho)$, we obtain $u^{\mathcal{I}}(\lambda) \geq$ $\lim _{L \rightarrow \infty} \mathbb{E}\left[L^{-1} \log U_{L}^{\omega, \mathcal{I}}(\lambda, \rho)\right]=-\lambda \rho+\phi^{\mathcal{I}}(1 / \rho)$. Therefore, optimising over $\rho$, we get $u^{\mathcal{I}}(\lambda) \geq \sup _{\rho \in(0,1]}\left\{-\lambda \rho+\phi^{\mathcal{I}}(1 / \rho)\right\}$.

To prove the reverse inequality, we note that an analogue of the concentration inequality (3.1.4) gives that there exists a $C>0$ such that, for all $L \in \mathbb{N}, \rho \in(0,1]$ and $\varepsilon>0$,

$$
\mathbb{P}\left(\frac{1}{L} \log U_{L}^{\omega, \mathcal{I}}(\lambda, \rho) \geq \mathbb{E}\left[\frac{1}{L} \log U_{L}^{\omega, \mathcal{I}}(\lambda, \rho)\right]+\varepsilon\right) \leq C \exp \left[-\varepsilon^{2} L / C(\alpha+\beta)^{2}\right] .
$$


Next, we define the event

$$
J(L)=\left\{\exists j \in\{1, \ldots, L\}: \frac{1}{L} \log U_{L}^{\omega, \mathcal{I}}(\lambda, j / L) \geq \mathbb{E}\left[\frac{1}{L} \log U_{L}^{\omega, \mathcal{I}}(\lambda, j / L)\right]+\varepsilon\right\},
$$

and abbreviate $E(L)=E\left[L^{-1} \log U_{L}^{\omega, \mathcal{I}}(\lambda)\right]$. Then we can write

$$
E(L) \leq \mathbb{E}\left(\left(\frac{1}{L} \log U_{L}^{\omega, \mathcal{I}}(\lambda)\right) 1_{J(L)}\right)+\mathbb{E}\left(\frac{1}{L} \log \left(\sum_{j=1}^{L} U_{L}^{\omega, \mathcal{I}}(\lambda, j / L)\right) 1_{[J(L)]^{c}}\right)
$$

Trivially, the quantity $L^{-1} \log U_{L}^{\omega, \mathcal{I}}(\lambda)$ can be bounded from above by $\alpha+\tilde{\kappa}(0)$ (recall (5.1.2)), uniformly in $L$ and $\omega$. Therefore, with the help of the inequality in (5.1.16), we see that the first term in the r.h.s. of (5.1.18) is bounded from above by $(\alpha+$ $\tilde{\kappa}(0)) C L \exp \left[-\varepsilon^{2} L /\left(C(\alpha+\beta)^{2}\right)\right]$, which tends to zero as $L \rightarrow \infty$. Moreover, for every $j \in\{1, \ldots, L\}$, a standard subadditivity argument gives that $\mathbb{E}\left(L^{-1} \log U_{L}^{\omega, \mathcal{I}}\right.$ $(\lambda, j / L)) \leq-\lambda j / L+\phi^{\mathcal{I}}(L / j)$. Therefore, on the event $[J(L)]^{c}$, we have that $L^{-1} \log$ $U_{L}^{\omega, \mathcal{I}}(\lambda, j / L) \leq-\lambda j / L+\phi^{\mathcal{I}}(L / j)+\varepsilon$ for all $j \in\{1, \ldots, L\}$. Thus, the second term in the r.h.s. of (5.1.18) is bounded from above by $(\log L) / L+\max _{\rho \in(0,1]}\left\{-\lambda \rho+\phi^{\mathcal{I}}(1 / \rho)\right\}+$ $\varepsilon$. Letting $L \rightarrow \infty$ and $\varepsilon \downarrow 0$, we obtain $\lim _{L \rightarrow \infty} E_{1}(L) \leq \max _{\rho \in(0,1]}\left\{-\lambda \rho+\phi^{\mathcal{I}}(1 / \rho)\right\}$, which is the reverse inequality we were after.

Since $\rho \mapsto \phi^{\mathcal{I}}(1 / \rho)$ is continuous and concave, we can apply the Fenchel-Legendre duality lemma (see Dembo and Zeitouni [2], Lemma 4.5.8), to obtain

$$
\phi^{\mathcal{I}}(\mu)=\inf _{\lambda \geq 0}\left\{\lambda / \mu+u^{\mathcal{I}}(\lambda)\right\}, \quad \mu \geq 1
$$

In the same spirit we have

$$
\begin{aligned}
& \tilde{\kappa}(\lambda)=\sup _{\rho \in(0,1]}\{-\lambda \rho+\hat{\kappa}(1 / \rho)\}, \quad \lambda \geq 0, \\
& \hat{\kappa}(\mu)=\inf _{\lambda \geq 0}\{\lambda / \mu+\tilde{\kappa}(\lambda)\}, \quad \mu \geq 1 .
\end{aligned}
$$

5.2. Positive and finite curvature of $u^{\mathcal{I}}$. In Propositions 5.1.1-5.1.3 we found that $u^{\mathcal{I}}$ is smooth and is the Fenchel-Legendre transform of $\phi^{\mathcal{I}}$. In Sect. 5.3 we will exploit these properties to obtain information on $\phi^{\mathcal{I}}$. To prepare for this, we first need to show the following. It is immediate from (5.1.1) that $\lambda \mapsto u^{\mathcal{I}}(\alpha, \beta ; \lambda)$ is convex. Lemma 5.2.1 and Assumption 5.2.2 below state that it has a strictly positive and finite curvature. To ease the notation, we suppress $\alpha, \beta$ from some of the expressions.

Lemma 5.2.1. For all $(\alpha, \beta, \lambda) \in \mathcal{L}_{u}, \partial^{2} u^{\mathcal{I}}(\alpha, \beta ; \lambda) / \partial \lambda^{2}>0$. 
Proof. It suffices to prove that for all $\left(\alpha, \beta, \lambda_{0}\right) \in \mathcal{L}_{u}$ there exist $C, \varepsilon>0$ such that, for all $\lambda \in I_{\varepsilon}\left(\lambda_{0}\right)=\left[\lambda_{0}-\varepsilon, \lambda_{0}+\varepsilon\right]$ and $L \geq 1$,

$$
\mathbb{E}\left(\left[E_{L}^{\omega, \mathcal{I}}\right]^{\otimes 2}\left(\left[h\left(\pi_{1}\right)-h\left(\pi_{2}\right)\right]^{2}\right)\right) \geq C L,
$$

where $E_{L}^{\omega, \mathcal{I}}$ is the expectation w.r.t. the law in (5.1.5), and $\lambda$ is suppressed from the notation.

Step 1. By Lemma 5.1.2(ii), we can assert that for all $T_{0} \in \mathbb{N}$ there exist $z_{0} \in(0,1)$ and $L_{0} \in \mathbb{N}$ such that, for all $L \geq L_{0}$ and $\lambda \in I_{\varepsilon}\left(\lambda_{0}\right)$,

$$
\mathbb{E}\left(P_{L}^{\omega, \mathcal{I}}\left(\left\{\sum_{k=1}^{l_{L}} \tau_{k} 1_{\left\{\tau_{k}>T_{0}\right\}} \geq z_{0} L\right\}\right)\right) \geq \frac{3}{4},
$$

where $\tau_{k}$ is the length of the $k^{t h}$ excursion. Similarly, by Lemma 5.1.2(i), there exists $M_{0} \in \mathbb{N}$ with $M_{0}>T_{0}$ and $L_{1} \in \mathbb{N}$ such that, for all $L \geq L_{1}$ and $\lambda \in I_{\varepsilon}\left(\lambda_{0}\right)$,

$$
\mathbb{E}\left(P_{L}^{\omega, \mathcal{I}}\left(\left\{\sum_{k=1}^{l_{L}} \tau_{k} 1_{\left\{\tau_{k} \leq M_{0}\right\}} \geq\left(1-\frac{z_{0}}{2}\right) L\right\}\right)\right) \geq \frac{3}{4} .
$$

Abbreviate $\Gamma_{0}=\left\{T_{0}+1, \ldots, M_{0}\right\} \times\{-1,+1\}$. Let $(j, \sigma) \in \Gamma_{0}$ and $L \geq L_{2}=$ $\max \left\{L_{0}, L_{1}\right\}$. Define

$$
\begin{aligned}
A(L) & =\left\{\sum_{k=1}^{l_{L}} \tau_{k} 1_{\left\{T_{0}<\tau_{k} \leq M_{0}\right\}} \geq \frac{z_{0}}{2} L\right\} \text { and } \\
B_{(j, \sigma)}(L) & =\left\{\sum_{k=1}^{l_{L}} \tau_{k} 1_{\left\{\tau_{k}=j, \sigma_{k}=\sigma\right\}} \geq \frac{z_{0}}{4\left(M_{0}-T_{0}\right)} L\right\},
\end{aligned}
$$

where $\sigma_{k}$ is the sign of the $k^{\text {th }}$ excursion. It follows from (5.2.2-5.2.3) that $\mathbb{E}$ $\left(P_{L}^{\omega, \mathcal{I}}(A(L))\right) \geq \frac{1}{2}$ and $A(L) \subset \cup_{(j, \sigma) \in \Gamma_{0}} B_{(j, \sigma)}(L)$. Since $\left|\Gamma_{0}\right|=2\left(M_{0}-t_{0}\right)$, for all $L \geq L_{2}$ and $\lambda \in I_{\varepsilon}\left(\lambda_{0}\right)$, there exists a $\left(j_{L}, \sigma_{L}\right) \in \Gamma_{0}$ such that

$$
\mathbb{E}\left(P_{L}^{\omega, \mathcal{I}}\left(B_{\left(j_{L}, \sigma_{L}\right)}(L)\right)\right) \geq \frac{1}{4\left(M_{0}-T_{0}\right)} .
$$

Step 2. Henceforth, we abbreviate $B^{L}=B_{\left(j_{L}, \sigma_{L}\right)}(L)$. We will show that the quantity

$$
\mathcal{H}^{L}=\mathbb{E}\left(\left[E_{L}^{\omega, \mathcal{I}}\right]^{\otimes 2}\left(\left[h\left(\pi_{1}\right)-h\left(\pi_{2}\right)\right]^{2} 1_{B^{L}}\left(\pi_{1}\right) 1_{B^{L}}\left(\pi_{2}\right)\right)\right)
$$

is bounded from below by $C L$ for some $C>0$, which will complete the proof of (5.2.1). For given $\pi$, we let

$$
T(\pi)=\left\{\left(T_{1}, T_{1}^{\prime}, \sigma_{1}\right), \ldots,\left(T_{l_{L}}, T_{l_{L}}^{\prime}, \sigma_{l_{L}}\right)\right\}
$$

denote the starting points, ending points and signs of the $l_{L}$ excursions of $\pi$ between 0 and $L$. For $r \in \mathbb{N}$, we set

$$
\mathcal{Z}_{r}^{L}=\left\{T(\pi): \pi \in B^{L}, l_{L}=r\right\},
$$


and we denote by $\mathcal{E}(T, \sigma)$ the set of excursions of length $T$ and sign $\sigma$. Futhermore, we write $\left(\varepsilon_{1}, \ldots, \varepsilon_{r}\right) \sim T$ as shorthand notation for $\left(\varepsilon_{1}, \ldots, \varepsilon_{r}\right) \in \mathcal{E}\left(T_{1}^{\prime}-T_{1}, \sigma_{1}\right) \times \cdots \times$ $\mathcal{E}\left(T_{r}^{\prime}-T_{r}, \sigma_{r}\right)$. With this notation, we can write the quantity in (5.2.6) as

$$
\mathcal{H}^{L}=\sum_{r, \tilde{r}} \sum_{T \in \mathcal{Z}_{r}^{L}} \sum_{\tilde{T} \in \mathcal{Z}_{\tilde{r}}^{L}} \mathbb{E}\left[\frac{1}{\left(Z_{L}^{\omega}\right)^{2}}\left(\prod_{s=1}^{r} \prod_{\tilde{s}=1}^{\tilde{r}} Z_{T, s}^{\omega} Z_{\tilde{T}, \tilde{s}}^{\omega}\right) R_{r, T, \tilde{r}, \tilde{T}}^{L}\right]
$$

with $Z_{L}^{\omega}$ the total partition sum,

$$
R_{r, T, \tilde{r}, \tilde{T}}^{L}=\sum_{\left(\varepsilon_{1}, \ldots, \varepsilon_{r}\right) \sim T} \sum_{\left(\tilde{\varepsilon}_{1}, \ldots, \tilde{\varepsilon}_{\tilde{r}}\right) \sim \tilde{T}} \prod_{s=1}^{r} \prod_{\tilde{s}=1}^{\tilde{r}} \frac{e^{-\lambda h\left(\varepsilon_{s}\right)} e^{-\lambda h\left(\tilde{\varepsilon}_{\tilde{s}}\right)}}{Z_{T, s} Z_{\tilde{T}, \tilde{s}}}\left[\sum_{s=1}^{r} h\left(\varepsilon_{s}\right)-\sum_{\tilde{s}=1}^{\tilde{r}} h\left(\tilde{\varepsilon}_{\tilde{s}}\right)\right]^{2}
$$

and (recall (2.3.3))

$$
\begin{aligned}
Z_{T, s}^{\omega} & =\sum_{\varepsilon_{s} \in \mathcal{E}(T, s)} e^{-\lambda h\left(\varepsilon_{s}\right)-H^{\omega, \mathcal{I}}\left(\varepsilon_{s}\right)}, \\
Z_{T, s} & =\sum_{\varepsilon_{s} \in \mathcal{E}(T, s)} e^{-\lambda h\left(\varepsilon_{s}\right)} .
\end{aligned}
$$

Note that $R_{r, T, \tilde{r}, \tilde{T}}^{L}$ does not depend on $\omega$.

Step 3. Putting

$$
X_{s}=h\left(\varepsilon_{s}\right), \quad \tilde{X}_{\tilde{s}}=h\left(\tilde{\varepsilon}_{\tilde{s}}\right), \quad t_{0}=z_{0} / 4 M_{0}\left(M_{0}-T_{0}\right),
$$

we note that in $R_{r, T, \tilde{r}, \tilde{T}}^{L}$ the random variables

$$
\left(X_{1}, \ldots, X_{r}, \tilde{X}_{1}, \ldots, \tilde{X}_{\tilde{r}}\right)
$$

are independent, and that the law of $X_{s}$ depends on $\left(T_{s}^{\prime}-T_{s}, \sigma_{s}\right)$. Since $(T, \tilde{T}) \in$ $\mathcal{Z}_{r}^{L} \times \mathcal{Z}_{\tilde{r}}^{L}$, there are at least $t_{0} L$ excursions of length $j_{L}$ and sign $\sigma_{L}$ in $T$ and $\tilde{T}$. Let $\left(s_{1}, \ldots, s_{t_{0} L}\right)$ and $\left(\tilde{s}_{1}, \ldots, \tilde{s}_{t_{0} L}\right)$ be the indices of the $t_{0} L$ first such excursions in $T$ and $\tilde{T}$, put

$$
Y_{r, T, \tilde{r}, \tilde{T}}^{L}=\sum_{s \in\{1, \ldots, r\} \backslash\left\{s_{1}, \ldots, s_{t_{0} L}\right\}} X_{s}-\sum_{\tilde{s} \in\{1, \ldots, \tilde{r}\} \backslash\left\{\tilde{s}_{1}, \ldots, \tilde{s}_{t_{0} L}\right\}} \tilde{X}_{\tilde{s}},
$$

and write (5.2.10) as

$$
R_{r, T, \tilde{r}, \tilde{T}}^{L}=E_{T, \tilde{T}}\left(\left[\sum_{k=1}^{t_{0} L} W_{k}+Y_{r, T, \tilde{r}, \tilde{T}}^{L}\right]^{2}\right),
$$

where $W_{k}=X_{s_{k}}-\tilde{X}_{\tilde{s}_{k}}$ and $E_{T, \tilde{T}}$ denotes expectation w.r.t. the law of (5.2.13). Clearly, $W=\left(W_{k}\right)_{k \in\left\{1, \ldots, t_{0} L\right\}}$ are i.i.d., symmetric and bounded random variables. Denote their variance by $v_{L}$. We can choose $T_{0}$ large enough so that the $W_{k}$ are not constant. Moreover, 
since the $W_{k}$ have only a finite number of laws, there exists an $a>0$ such that $v_{L}>a$ for all $\lambda \in I_{\varepsilon}\left(\lambda_{0}\right)$ and $L \geq L_{2}$.

Step 4. At this stage, we may assume without loss of generality that $P_{T, \tilde{T}}\left(Y_{r, T, \tilde{r}, \tilde{T}}^{L} \geq\right.$ $0) \geq \frac{1}{2}$. Then (5.2.15) gives

$$
R_{r, T, \tilde{r}, \tilde{T}}^{L} \geq P_{T, \tilde{T}}\left(Y_{r, T, \tilde{r}, \tilde{T}}^{L} \geq 0\right) \frac{1}{2} E_{T, \tilde{T}}\left(\left[\sum_{k=1}^{t_{0} L} W_{k}\right]^{2}\right) \geq \frac{1}{4} E_{\left(j_{L}, \sigma_{L}\right)}\left(\left[\sum_{k=1}^{t_{0} L} W_{k}\right]^{2}\right),
$$

where $E_{\left(j_{L}, \sigma_{L}\right)}$ is expectation w.r.t. the law of $W$. Since the $W_{k}$ take only values smaller than $2 M_{0}$, their third moments are bounded by some finite $N$ uniformly in $\lambda \in I_{\varepsilon}\left(\lambda_{0}\right)$ and $(j, \sigma) \in \Gamma_{0}$. Therefore we can apply the Berry-Esseen theorem and, writing $\xi(u)=$ $P(\mathcal{N}(0,1) \leq u), u \in \mathbb{R}$ with $N(0,1)$ a standard normal random variable, can assert that, for all $u \in \mathbb{R}, \lambda \in I_{\varepsilon}\left(\lambda_{0}\right)$ and $(j, \sigma) \in \Gamma_{0}$,

$$
\left|P_{(j, \sigma)}\left(\sum_{k=1}^{t_{0} L} W_{k} \leq u \sqrt{t_{0} L v_{L}}\right)-\xi(u)\right| \leq \frac{3 N}{a^{3 / 2} \sqrt{t_{0} L}},
$$

where $P_{(j, \sigma)}$ is the law of $W$ when $\left(j_{L}, \sigma_{L}\right)=(j, \sigma)$. Taking the restriction of the r.h.s. of (5.2.16) to the event $K=\left\{\sum_{k=1}^{t_{0} L} W_{k} / \sqrt{t_{0} L v_{L}} \in[1,2]\right\}$, we obtain

$$
R_{r, T, \tilde{r}, \tilde{T}}^{L} \geq \frac{v_{L} t_{0} L}{4} P_{(j, \sigma)}(K) \geq \frac{a t_{0} L}{4}\left(\xi(2)-\xi(1)-\frac{6 N}{a^{3 / 2} \sqrt{t_{0} L}}\right),
$$

which implies that $R_{r, T, \tilde{r}, \tilde{T}}^{L} \geq t_{0}^{\prime} L$ for $L$ large enough and some $t_{0}^{\prime}>0$. Recalling (5.2.9), we can now estimate

$$
\mathcal{H}^{L} \geq t_{0}^{\prime} L \mathbb{E}\left(\left[P_{L}^{\omega, \mathcal{I}}\right]^{\otimes 2}\left(B^{L}\right)\right) \geq t_{0}^{\prime} L / 4\left(M_{0}-T_{0}\right),
$$

which yields (5.2.1) with $C=t_{0}^{\prime} L / 4\left(M_{0}-T_{0}\right)$.

The following assumption will be needed in Sects. 5.3-5.5.

Assumption 5.2.2. For all $(\alpha, \beta) \in \operatorname{CONE}$ and $\lambda>0$ there exist $C(\lambda)>0$ and $\delta_{0}>0$ such that, for all $\delta \in\left(0, \delta_{0}\right]$,

$$
u^{\mathcal{I}}(\lambda-\delta)+u^{\mathcal{I}}(\lambda+\delta)-2 u^{\mathcal{I}}(\lambda) \leq C(\lambda) \delta^{2} .
$$

Although we are not able to prove this assumption, we believe it to be true for the following reason. First, as a consequence of Proposition 5.1.1, we have that, for all $(\alpha, \beta) \in$ CONE, $\lambda \mapsto u(\alpha, \beta ; \lambda)$ is infinitely differentiable on the set $\{\lambda \in[0, \infty): u(\alpha, \beta ; \lambda)>$ $\tilde{\kappa}(\lambda)\}$. Since $\lambda \mapsto \tilde{\kappa}(\lambda)$ is infinitely differentiable on $[0, \infty)$, this implies that $\lambda \mapsto$ $u(\alpha, \beta ; \lambda)$ is infinitely differentiable on the interior of the set $\{\lambda \in[0, \infty): u(\alpha, \beta ; \lambda)=$ $\tilde{\kappa}(\lambda)\}$. Thus, the assumption only concerns the values of $\lambda$ located at the boundary of the latter. For these values, proving the assumption amounts to proving the reverse of inequality (5.2.1), i.e., showing that the variance of the number of horizontal steps made by the polymer of length $L$ is of order $L$, which we may reasonably expect to be true. In Remark 5.3.3 we give a weaker alternative to Assumption 5.2.2. 
5.3. Smoothness of $\phi^{\mathcal{I}}$ in its localized phase. Having collected in Sects. 5.1-5.2 some key properties of the dual free energy $u^{\mathcal{I}}$, we are now ready to look at what these imply for $\phi^{\mathcal{I}}$. We begin by showing that $\phi^{\mathcal{I}}$ is strictly concave.

Lemma 5.3.1. Let

$$
D(\delta)=\frac{1}{2} \phi^{\mathcal{I}}\left(\frac{1}{\rho_{0}+\delta}\right)+\frac{1}{2} \phi^{\mathcal{I}}\left(\frac{1}{\rho_{0}-\delta}\right)-\phi^{\mathcal{I}}\left(\frac{1}{\rho_{0}}\right) .
$$

Then, for all $(\alpha, \beta) \in \mathrm{CONE}$ and $\rho_{0} \in(0,1)$ there exist $C>0$ and $\delta_{0}>0$ such that, for all $\delta \in\left(0, \delta_{0}\right]$,

$$
D(\delta) \leq-C \delta^{2}
$$

This inequality implies the strict concavity of $\rho \mapsto \phi^{\mathcal{I}}(1 / \rho)$ on $(0,1]$.

Proof. Lemma 5.2.1 states the strict convexity of $\lambda \mapsto u^{\mathcal{I}}(\lambda)$, which implies the uniqueness of the maximiser in the variational formula (5.1.19), i.e., there exists a unique $\lambda_{0}=\lambda_{0}(\rho) \geq 0$ such that $\phi^{\mathcal{I}}\left(1 / \rho_{0}\right)=\lambda_{0} \rho_{0}+u^{\mathcal{I}}\left(\lambda_{0}\right)$. Let $x>0$. By picking $\lambda=\lambda_{0}-x \delta$ in (5.1.19) with $\mu=1 /\left(\rho_{0}+\delta\right)$, and $\lambda=\lambda_{0}+x \delta$ in (5.1.19) with $\mu=1 /\left(\rho_{0}-\delta\right)$, we obtain

$$
\begin{aligned}
D(\delta) \leq & \frac{1}{2}\left[\left(\lambda_{0}-x \delta\right)\left(\rho_{0}+\delta\right)+u^{\mathcal{I}}\left(\lambda_{0}-x \delta\right)\right] \\
& +\frac{1}{2}\left[\left(\lambda_{0}+x \delta\right)\left(\rho_{0}-\delta\right)+u^{\mathcal{I}}\left(\lambda_{0}+x \delta\right)\right]-\lambda_{0} \rho_{0}-u^{\mathcal{I}}\left(\lambda_{0}\right) \\
= & -x \delta^{2}+\frac{1}{2}\left[u^{\mathcal{I}}\left(\lambda_{0}-x \delta\right)+u^{\mathcal{I}}\left(\lambda_{0}+x \delta\right)-2 u^{\mathcal{I}}\left(\lambda_{0}\right)\right] .
\end{aligned}
$$

Picking $x=1 / 2 C\left(\lambda_{0}\right)$, with $C\left(\lambda_{0}\right)$ the constant in Assumption 5.2.2, we see that (5.3.3) implies, for $0<\delta<2 C\left(\lambda_{0}\right) \delta_{0}$,

$$
D(\delta) \leq-x \delta^{2}+C\left(\lambda_{0}\right) x^{2} \delta^{2}=-\delta^{2} / 4 C\left(\lambda_{0}\right),
$$

which proves (5.3.2). To prove the claim made below (5.3.2), pick $1 \leq u<v$ and consider (5.3.1) at the point $\rho_{0}=(u+v) / 2$. Then, by (5.3.1-5.3.2), there exists a $0<\delta<(v-u) / 2$ such that

$$
\frac{\phi^{\mathcal{I}}\left(\frac{1}{\rho_{0}+\delta}\right)-\phi^{\mathcal{I}}\left(\frac{1}{\rho_{0}}\right)}{\delta}<\frac{\phi^{\mathcal{I}}\left(\frac{1}{\rho_{0}}\right)-\phi^{\mathcal{I}}\left(\frac{1}{\rho_{0}-\delta}\right)}{\delta} .
$$

Since $v>\rho_{0}+\delta>\rho_{0}-\delta>u$, it follows that

$$
\left.\frac{\partial^{-} \phi^{\mathcal{I}}}{\partial \rho}\left(\frac{1}{\rho}\right)\right|_{\rho=v} \leq \text { 1.h.s. }(5.3 .5)<\text { r.h.s. }(5.3 .5) \leq\left.\frac{\partial^{+} \phi^{\mathcal{I}}}{\partial \rho}\left(\frac{1}{\rho}\right)\right|_{\rho=u},
$$

with - and + denoting the left- and the right-derivative.

We are now ready to prove that $\phi^{\mathcal{I}}$ is smooth. Let

$$
\mathcal{L}_{\phi}=\left\{(\alpha, \beta, \mu)=\operatorname{CONE} \times[1, \infty): \phi^{\mathcal{I}}(\alpha, \beta ; \mu)>\hat{\kappa}(\mu)\right\},
$$

i.e., the region where the single linear interface model is localized.

Proposition 5.3.2. $(\alpha, \beta, \mu) \mapsto \phi^{\mathcal{I}}(\alpha, \beta ; \mu)$ is infinitely differentiable on $\mathcal{L}_{\phi}$. 
Proof. Let $(\alpha, \beta, \mu) \in \mathcal{L}_{\phi}$. Lemma 5.2.1 states the strict convexity of $\lambda \mapsto u^{\mathcal{I}}(\lambda)$ on $\{\lambda: u(\lambda)>\tilde{\kappa}(\lambda)\}$ and it can be shown that $\lambda \mapsto \tilde{\kappa}(\lambda)$ is strictly convex on $[0, \infty)$. This entails that $\lambda \mapsto u^{\mathcal{I}}(\lambda)$ is strictly convex on $[0, \infty)$. Therefore, the variational formula in (5.1.19) attains its maximum at a unique point $\lambda(\mu) \geq 0$, so that the variational formula in (5.1.14) allows us to write

$$
\phi^{\mathcal{I}}(\mu)=\lambda(\mu) / \mu+\sup _{\rho \in(0,1]}\left\{-\lambda(\mu) \rho+\phi^{\mathcal{I}}(1 / \rho)\right\}
$$

after which the strict concavity of $\rho \mapsto \phi^{\mathcal{I}}(1 / \rho)$ (recall Lemma 5.3.1) implies that this supremum is attained uniquely at $\rho=1 / \mu$. Since $\phi^{\mathcal{I}}(\rho) \geq \hat{\kappa}(\rho)$ for all $\rho$, and $\phi^{\mathcal{I}}(\mu)>$ $\hat{\kappa}(\mu)$, the variational formula in (5.1.20) allows us to write $u^{\mathcal{I}}(\lambda(\mu))>\tilde{\kappa}(\lambda(\mu))$, and therefore $(\alpha, \beta, \lambda(\mu)) \in \mathcal{L}_{u}$.

Next, let

$$
\mathcal{S}=\left\{(\alpha, \beta, \mu, \lambda) \in \operatorname{CONE} \times[1, \infty) \times[0, \infty):(\alpha, \beta, \mu) \in \mathcal{L}_{\phi},(\alpha, \beta, \lambda) \in \mathcal{L}_{u}\right\}
$$

and define $\Upsilon_{1}$ as

$$
\Upsilon_{1}:(\alpha, \beta, \mu, \lambda) \in \mathcal{S} \mapsto \frac{\partial\left(\lambda / \mu+u^{\mathcal{I}}(\lambda)\right)}{\partial \lambda} .
$$

We want to apply the implicit function theorem in Bredon [1], Chapter II, Theorem 1.5, to $\Upsilon_{1}$. This requires checking three properties:

(i) $\Upsilon_{1}$ is infinitely differentiable on $\mathcal{S}$.

(ii) For all $(\alpha, \beta, \mu) \in \mathcal{L}_{\phi}, \lambda(\mu)$ is the unique $\lambda \in[1, \infty)$ such that $(\alpha, \beta, \lambda) \in \mathcal{L}_{u}$ and $\Upsilon_{1}(\alpha, \beta, \mu, \lambda(\mu))=0$.

(iii) For all $(\alpha, \beta, \mu) \in \mathcal{L}_{\phi}, \frac{\partial \Upsilon_{1}}{\partial \lambda}(\alpha, \beta, \mu, \lambda(\mu)) \neq 0$.

Property (i) holds because $u^{\mathcal{I}}$ is infinitely differentiable on $\mathcal{L}_{u}$ (by Proposition 5.1.1). Property (ii) holds because $\lambda \mapsto u^{\mathcal{I}}(\lambda)$ is strictly convex (by Lemma 5.2.1). Moreover, Lemma 5.2.1 gives that

$$
\frac{\partial \Upsilon_{1}}{\partial \lambda}(\alpha, \beta, \mu, \lambda(\mu))=\frac{\partial^{2} u^{\mathcal{I}}}{\partial \lambda^{2}}(\alpha, \beta, \lambda(\mu))>0,
$$

so property (iii) holds too. We can therefore indeed use the implicit function theorem, obtaining that $(\alpha, \beta, \mu) \mapsto \lambda(\mu)$ and $(\alpha, \beta, \mu) \mapsto \phi^{\mathcal{I}}(\alpha, \beta ; \mu)$ are infinitely differentiable on $\mathcal{L}_{\phi}$.

Remark 5.3.3. Assumption 5.2.2 can be weakened. Namely, instead of assuming finite curvature of $\lambda \mapsto u(\alpha, \beta ; \lambda)$, we may assume strict concavity of $\mu \mapsto \mu \phi^{\mathcal{I}}(\mu)$ (which is already known to be concave). This strict concavity (which is implied by Assumption 5.2.2, Lemma 5.3.1 and (5.4.1)) is sufficient to guarantee, in the proof of Proposition 5.3.2, that $\lambda(\mu)$ in (5.3.8) is unique and satisfies $(\alpha, \beta, \lambda(\mu)) \in \mathcal{L}_{\mu}$. The latter in turn is enough to carry out the rest of the proof. 
5.4. Smoothness of $\psi_{A B}$ in its localized phase. In this section we transport the properties of $\phi^{\mathcal{I}}$ obtained in Sect. 5.3 to $\psi_{A B}$. We begin with some elementary observations. Fix $(\alpha, \beta) \in$ CONE and recall (2.3.4). By Lemma 5.3.1 and Lemma 2.2.1(ii), for all $a \geq 2$, $(c, b) \mapsto c \phi^{\mathcal{I}}(c / b)$ and $(c, b) \mapsto(a-c) \kappa(a-c, 1-b)$ are strictly concave on $\operatorname{DOM}(a)$. Consequently, for all $a \geq 2$, the supremum of the variational formula in (2.3.6) is attained at a unique pair $(c, b) \in \operatorname{DOM}(a)$ (use that $\operatorname{DOM}(a)$ is a convex set).

Next, note that Lemma 5.3.1 and Proposition 5.3.2 imply that for all $\left(\alpha, \beta, \rho_{0}\right) \in \mathcal{L}_{\phi}$ there exists a $C>0$ such that

$$
\frac{\partial^{2}}{\partial \rho^{2}}\left[\rho \phi^{\mathcal{I}}(\rho)\right]\left(\rho_{0}\right)=\frac{1}{\rho_{0}^{3}} \frac{\partial^{2}}{\partial \rho^{2}}\left[\phi^{\mathcal{I}}(1 / \rho)\right]\left(\frac{1}{\rho_{0}}\right) \leq-C
$$

Let

$$
\mathcal{L}_{\psi}=\left\{(\alpha, \beta, a) \in \mathrm{CONE} \times[2, \infty): \psi_{A B}(\alpha, \beta ; a)>\varpi\right\}
$$

i.e., the region where $\psi_{A B}$ is localized. Our main result in this section is the following.

Proposition 5.4.1. $(\alpha, \beta, a) \mapsto \psi_{A B}(\alpha, \beta ; a)$ is infinitely differentiable on $\mathcal{L}_{\psi}$.

Proof. Define

$$
\mathcal{L}_{\alpha, \beta, a}=\left\{(c, b) \in \operatorname{DOM}(a): \phi^{\mathcal{I}}(\alpha, \beta ; c / b)>\hat{\kappa}(c / b)\right\} .
$$

As noted above, the variational formula in (2.3.6) attains its maximum at a unique pair $(c(\alpha, \beta ; a), b(\alpha, \beta ; a)) \in \operatorname{DOM}(a)$. We write $(c(a), b(a))$, suppressing $(\alpha, \beta)$ from the notation. Since $(\alpha, \beta) \in \mathcal{L}$ (recall (1.3.1)), Lemma 2.2.2 (iv) and Proposition 2.3.4 imply that $(c(a), b(a)) \in \mathcal{L}_{\alpha, \beta, a}$. Let

$$
F(c, b)=c \phi(c / b), \quad \tilde{F}(c, b)=(a-c) \kappa(a-c, 1-b),
$$

and denote by $\left\{F_{c}, F_{b}, F_{c c}, F_{c b}, F_{b b}\right\}$ the partial derivatives of order 1 and 2 of $F$ with respect to the variables $c$ and $b$ (and similarly for $\tilde{F}$ ). By the strict concavity of $(c, b) \mapsto$ $F(c, b)+\tilde{F}(c, b)$ in $\operatorname{DOM}(a)$, we know that $(c(a), b(a))$ is also the unique pair in $\mathcal{L}_{\alpha, \beta, a}$ at which $F_{c}+\tilde{F}_{c}=0$ and $F_{b}+\tilde{F}_{b}=0$.

We need to show that $(c(a), b(a))$ is infinitely differentiable w.r.t. $(\alpha, \beta, a)$. To that aim we again use the implicit function theorem. Define

$$
\mathcal{R}=\left\{(\alpha, \beta, a, c, b):(\alpha, \beta, a) \in \mathcal{L}_{\psi},(c, b) \in \mathcal{L}_{\alpha, \beta, a}\right\}
$$

and

$$
\Upsilon_{2}:(\alpha, \beta, a, c, b) \in \mathcal{R} \mapsto\left(F_{c}+\tilde{F}_{c}, F_{b}+\tilde{F}_{b}\right)
$$

Let $J_{2}$ be the Jacobian determinant of $\Upsilon_{2}$ as a function of $(c, b)$. Applying the implicit function theorem to $\Upsilon_{2}$ requires checking three properties:

(i) $\Upsilon_{2}$ is infinitely differentiable on $\mathcal{R}$.

(ii) For all $(\alpha, \beta, a) \in \mathcal{L}_{\psi},(c(a), b(a))$ is the only pair in $\mathcal{L}_{\alpha, \beta, a}$ satisfying $\Upsilon_{2}=0$.

(iii) For all $(\alpha, \beta, a) \in \mathcal{L}_{\psi}, J_{2} \neq 0$ in $(c(a), b(a))$. 
As explained below (5.4.4), property (ii) holds. Proposition 5.3.2 and Lemma 2.2.2 (ii) show that also property (i) holds. Computing the Jacobian determinant $J_{2}$, we get

$$
J_{2}=\left(F_{c c}+\tilde{F}_{c c}\right)\left(F_{b b}+\tilde{F}_{b b}\right)-\left(F_{c b}+\tilde{F}_{c, b}\right)^{2} .
$$

Since $F_{c c} F_{b b}-F_{c b}^{2}=0, F_{b b}=\mu^{2} F_{c c}$ and $F_{c b}=\mu F_{c c},(5.4 .7)$ becomes

$$
J_{2}=\tilde{F}_{c c} \tilde{F}_{b b}-\tilde{F}_{c b}^{2}+F_{c c}\left[\tilde{F}_{b b}+2 \mu \tilde{F}_{c b}+\mu^{2} \tilde{F}_{c c}\right]
$$

By the concavity of $c \mapsto F(c, b)$ and $c \mapsto \tilde{F}(c, b)$, we have $F_{c c} \leq 0$ and $\tilde{F}_{c c} \leq 0$. Moreover, by the concavity of $(c, b) \mapsto \tilde{F}(c, b)$, its Hessian matrix necessarily has two non-positive eigenvalues. Therefore, the determinant of this matrix is non-negative, i.e., $\tilde{F}_{c c} \tilde{F}_{b b}-\tilde{F}_{c b}^{2} \geq 0$. This, together with the inequality $\tilde{F}_{c c} \leq 0$, implies that $\mu \mapsto$ $\tilde{F}_{b b}+2 \mu \tilde{F}_{c b}+\mu^{2} \tilde{F}_{c c}$ is non-positive on $\mathbb{R}$. Hence $J_{2} \geq 0$.

Lemma 5.4.2. $\tilde{F}_{c c} \tilde{F}_{b b}-\tilde{F}_{c b}^{2}>0$.

Proof. The strict inequality can be checked with MAPLE. In [7], an explicit variational formula is given for the entropy function in (2.2.2), which is easily implemented.

It follows from Lemma 5.4.2 that $J_{2}>0$, which proves property (iii). We know from Lemma 2.2.1 (ii) and Proposition 5.3.2 that $\tilde{F}$ and $F$ are infinitely differentiable on $\operatorname{DOM}(a)$ for all $a \in[2, \infty)$. Hence, the claim indeed follows the implicit function theorem.

We close this section with the following observations needed in Sect. 5.5.

Lemma 5.4.3. Fix $(\alpha, \beta) \in$ CONE.

(i) For all $k, l \in\{A, B\}, a \mapsto \psi_{k l}(a)$ is strictly concave on $[2, \infty)$.

(ii) For all $k, l \in\{A, B\}$ with $k l \neq B B, \lim _{a \rightarrow \infty} a \psi_{k l}(a)=\infty$.

(iii) For all $k, l \in\{A, B\}, \lim _{a \rightarrow \infty} \partial\left[a \psi_{k l}(a)\right] / \partial a \leq 0$.

Proof. (i) This is a straightforward consequence of the observations made at the beginning of this section, together with the strict concavity of $\mu \mapsto \mu \phi^{\mathcal{I}}(\mu)$ proved in Lemma 5.3.1.

(ii) Because $\psi_{A B} \geq \psi_{A A}$, it suffices to consider $k l \in\{A A, B A\}$. For $k l=A A$, the claim is immediate from Lemma 2.2.1(iii) and (2.3.1). For $k l=B A$, we use the fact that $\phi^{\mathcal{I}}(\mu) \geq \hat{\kappa}(\mu)$ (recall (2.3.8)) in combination with the variational formula of Lemma 2.3.2 with $c=a-\frac{3}{2}$ and $b=\frac{1}{2}$. This gives

$$
a \psi_{B A}(a) \geq \frac{1}{2}(2 a-3) \hat{\kappa}(2 a-3)+\frac{3}{2}\left[\kappa\left(\frac{3}{2}, \frac{1}{2}\right)+\frac{1}{2}(\beta-\alpha)\right],
$$

which yields the claim because $\mu \hat{\kappa}(\mu) \sim \log \mu$ as $\mu \rightarrow \infty$ by Lemma 2.2.2(iii).

(iii) Since, for all $k, l \in\{A, B\}, \psi_{A B} \geq \psi_{k l}$ and $a \mapsto a \psi_{k l}(a)$ is concave, it suffices to prove that $\limsup _{a \rightarrow \infty} \psi_{A B}(a) \leq 0$. The latter is immediate from the variational formula in (2.3.6) and the fact that $\lim _{a \rightarrow \infty} \phi^{\mathcal{I}}(a)=0$ (Lemma 4.2.6(i)) and $\lim _{a \rightarrow \infty} \kappa(a, 1)=0((2.2 .3))$. 
5.5. Smoothness of $f$ on $\mathcal{L}$. We begin by proving the uniqueness of the maximisers in the variational formula in (2.1.11). For $(\alpha, \beta) \in \operatorname{CONE}, p \in(0,1)$ and $\left(\rho_{k l}\right) \in \mathcal{R}(p)$, let (recall (2.1.9))

$$
\begin{aligned}
f_{\left(\rho_{k l}\right)} & =\sup _{\left(a_{k l}\right) \in \mathcal{A}} V\left(\left(\rho_{k l}\right),\left(a_{k l}\right)\right), \\
\mathcal{O}_{\left(\rho_{k l}\right)} & =\left\{k l \in\{A, B\}^{2}: \rho_{k l}>0\right\}, \\
\mathcal{R}^{f}(p) & =\left\{\left(\rho_{k l}\right) \in \mathcal{R}(p): f=f_{\left(\rho_{k l}\right)}\right\}, \\
\mathcal{P}(p) & =\bigcup_{\left(\rho_{k l}\right) \in \mathcal{R}^{f}(p)} \mathcal{O}_{\left(\rho_{k l}\right)} .
\end{aligned}
$$

Proposition 5.5.1. (i) For every $(\alpha, \beta) \in$ CONE, $p \in(0,1)$ and $\rho=\left(\rho_{k l}\right) \in \mathcal{R}(p)$, there exists a unique family $a^{\rho}=\left(a_{k l}^{\rho}\right)_{k l \in \mathcal{O}_{\rho}} \in \mathcal{A}$ satisfying

$$
f_{\rho}=\frac{\sum_{k l \in \mathcal{O}_{\rho}} \rho_{k l} a_{k l}^{\rho} \psi_{k l}\left(a_{k l}^{\rho}\right)}{\sum_{k l \in \mathcal{O}_{\rho}} \rho_{k l} a_{k l}^{\rho}}=V\left(\rho, a^{\rho}\right)
$$

(ii) For every $(\alpha, \beta) \in \mathrm{CONE}$ and $p \in(0,1), \mathcal{R}^{f}(p) \neq \emptyset$, and there exists a unique

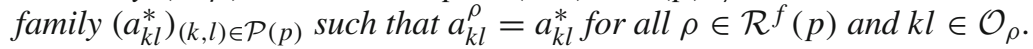

Proof. Recall Theorem 2.1.1. (i) The case $\rho_{B B}=1$ is trivial. In that case we have $f_{\rho}=\sup _{a_{B B} \geq 2} \psi_{B B}\left(a_{B B}\right)=\psi_{B B}\left(a^{*}\right)=\frac{1}{2} \beta+\varpi$ (by Lemma 2.2.1(iv)), and so $a_{B B}^{\rho}=$ $a^{*}=\frac{5}{2}$. Therefore assume that $\rho_{B B}<1$. Then at least one pair $k_{1} l_{1} \in\{A A, A B, B A\}$ satisfies $\rho_{k_{1} l_{1}}>0$, and since $\lim _{u \rightarrow \infty} u \psi_{k_{1} l_{1}}(u)=\infty$ by Lemma 5.4 .3 (ii), we have $f_{\rho}>0$. The latter is needed in what follows.

To prove existence of $a^{\rho}$, for $R>0$ let

$$
f_{\rho, R}=\sup _{a \in[2, R]} V(\rho, a)
$$

We prove that for $R$ large enough the supremum in (5.5.2) is attained in $[2, R]^{\mathcal{O}_{\rho}}$, i.e., $f_{\rho}=f_{\rho, R}$. Indeed, for $a \in \mathcal{A}, \rho \in \mathcal{R}(p)$ and $k_{2} l_{2} \in\{A, B\}^{2}$ we have (recall (2.1.9))

$$
\frac{\partial V}{\partial a_{k_{2} l_{2}}}(\rho, a)=\frac{\rho_{k_{2} l_{2}}}{\sum_{k l} \rho_{k l} a_{k l}}\left\{\left.\frac{\partial\left[u \psi_{k_{2} l_{2}}(u)\right]}{\partial u}\right|_{u=a_{k_{2} l_{2}}}-V(\rho, a)\right\} .
$$

Moreover, for every $k l \in\{A, B\}^{2}, u \mapsto u \psi_{k l}(u)$ is strictly concave and $u \mapsto$ $\partial\left[u \psi_{k l}(u)\right] / \partial u$ is strictly decreasing (by Lemma 5.4.3(i)) and converges to a limit $\leq 0$ as $u \rightarrow \infty$ (by Lemma 5.4.3(iii)). Pick $R>0$ large enough so that $\partial\left[u \psi_{k l}(u)\right] / \partial u \leq f_{\rho} / 2$ for all $u \geq R$ and $k l \in\{A, B\}^{2}$. We will show that $f_{\rho}>f_{\rho, R}$ implies that $V(\rho, a) \leq$ $\max \left\{f_{\rho} / 2, f_{\rho, R}\right\}$ for all $a \in \mathcal{A} \backslash[2, R]^{\mathcal{O}_{\rho}}$, and this will provide a contradiction.

To achieve the latter, assume that $A A \in \mathcal{O}_{\rho}$ and consider, for instance, $a \in \mathcal{A}$ such that $a_{A A}>R$ and $a_{k l} \leq R$ for $k l \in \mathcal{O}_{\rho} \backslash\{A A\}$. Fix $x \geq R$ and denote by $a^{x}$ the element of $\mathcal{O}_{\rho}$ given by $a_{A A}^{x}=x$ and $a_{k l}^{x}=a_{k l}, k l \in \mathcal{O}_{\rho} \backslash\{A A\}$. Since $a^{R} \in[2, R]^{\mathcal{O}_{\rho}}$, we have $V\left(\rho, a^{R}\right) \leq f_{\rho, R}<f_{\rho}$ and

$$
V\left(\rho, a^{x}\right)-V\left(\rho, a^{R}\right)=\int_{R}^{x} \frac{\partial V}{\partial a_{A A}}\left(\rho, a^{u}\right) d u .
$$


Since, by (5.5.4), the sign of $\left(\partial V / \partial a_{A A}\right)\left(\rho, a^{u}\right)$ is equal to the sign of $\partial\left[u \psi_{A A}(u)\right] / \partial u-$ $V\left(\rho, a^{u}\right)$, it follows that $V\left(\rho, a^{x}\right)$ decreases with $x$ whenever $V\left(\rho, a^{x}\right) \geq f_{\rho} / 2$. Since $V\left(\rho, a^{R}\right)<f_{\rho}$, we therefore have $V\left(\rho, a^{x}\right) \leq \max \left\{f_{\rho} / 2, f_{\rho, R}\right\}$ for all $x \geq R$ and, consequently, $V(\rho, a) \leq \max \left\{f_{\rho} / 2, f_{\rho, R}\right\}$. Therefore the supremum of (5.5.2) is attained in $[2, R]^{\mathcal{O}_{\rho}}$.

The uniqueness of $a^{\rho}$ realising $f_{\rho}=V\left(\rho, a^{\rho}\right)$ follows from (5.5.4), because for each $k l \in\{A, B\}^{\mathcal{O}_{\rho}}$ we must have $\left(\partial V / \partial a_{k l}\right)\left(\rho, a^{\rho}\right)=0$. This means that for each $k l \in \mathcal{O}_{\rho}$ we must have

$$
\left.\frac{\partial\left[u \psi_{k l}(u)\right]}{\partial u}\right|_{u=a_{k l}^{\rho}}=V\left(\rho, a^{\rho}\right)=\sup _{a \in \mathcal{A}} V(\rho, a),
$$

and, since $u \mapsto u \psi_{k l}(u)$ is strictly concave (by Lemma 5.4.3(i)), there is only one such $a_{k l}$ for each $k l \in \mathcal{O}_{\rho}$.

(ii) As shown in [7], Proposition 3.2.1, $\rho \mapsto f_{\rho}$ is continuous on $\mathcal{R}(p)$. Therefore, the compactness of $\mathcal{R}(p)$ entails $\mathcal{R}^{f}(p) \neq \emptyset$. Consider $\left(\rho_{1}, \rho_{2}\right) \in \mathcal{R}^{f}(p)$ and $k l \in$ $\mathcal{O}_{\rho_{1}} \cap \mathcal{O}_{\rho_{2}}$. Then (5.5.4) also gives

$$
\left.\frac{\partial\left[u \psi_{k l}(u)\right]}{\partial u}\right|_{u=a_{k l}^{\rho_{1}}}=f=\left.\frac{\partial\left[u \psi_{k l}(u)\right]}{\partial u}\right|_{u=a_{k l}^{\rho_{2}}},
$$

which, by the strict concavity of $u \mapsto u \psi_{k l}(u)$, implies that $a_{k l}^{\rho_{1}}=a_{k l}^{\rho_{2}}$.

We are now ready to prove the smoothness of $f$ on $\mathcal{L}$. Because of the inequalities $\psi_{A A} \geq \psi_{B B}$ and $\psi_{A B} \geq \psi_{B A}$, the concavity of $a \mapsto a \psi_{A A}(a)$ and $a \mapsto a \psi_{A B}(a)$ implies that the variational problem in (2.1.11) reduces to the matrices $\left\{M_{\gamma}, \gamma \in C\right\}$, with $M_{\gamma}$ the matrix and $C$ the set defined in (2.1.8). Write $V\left(\gamma, a_{A B}, a_{A A}\right)$ for the quantity $V\left(M_{\gamma},\left(a_{A B}, a_{A A}, 0,0\right)\right)$ defined in (2.1.9), put $\gamma^{*}=\max C$ and let $\left(x^{*}(\alpha, \beta)\right.$, $\left.y^{*}(\alpha, \beta)\right)$ be the unique maximisers $\left(a_{A B}^{*}, a_{A A}^{*}\right)$ defined in Proposition 5.5.1. By differentiating the quantity $V\left(\gamma, x^{*}, y^{*}\right)$ with respect to $\gamma$, we easily get that $\mathcal{R}^{f}(p)$ contains only the matrix $M_{\gamma^{*}}$. Thus, we have the equality

$$
f(\alpha, \beta)=V\left(\gamma^{*}, x^{*}, y^{*}\right)=\frac{\gamma^{*} x^{*} \psi_{A B}\left(x^{*}\right)+\left(1-\gamma^{*}\right) y^{*} \kappa\left(y^{*}, 1\right)}{\gamma^{*} x^{*}+\left(1-\gamma^{*}\right) y^{*}} .
$$

Since $(\alpha, \beta) \in \mathcal{L}$, we have $\psi_{A B}\left(x^{*}\right)>\varpi$ and therefore $\left(\alpha, \beta, x^{*}\right) \in \mathcal{L}_{\psi}$. To show that $f$ is infinitely differentiable on $\mathcal{L}$, we once more use the implicit function theorem. For that we define

$$
\mathcal{N}=\left\{(\alpha, \beta, x, y):(\alpha, \beta) \in \mathcal{L},(\alpha, \beta, x) \in \mathcal{L}_{\psi}, y>2\right\}
$$

and

$$
\Upsilon_{3}:(\alpha, \beta, x, y) \in \mathcal{N} \mapsto\left(\frac{\partial V}{\partial x}\left(\gamma^{*}, x, y\right), \frac{\partial V}{\partial y}\left(\gamma^{*}, x, y\right)\right) .
$$

Let $J_{3}$ be the Jacobian determinant of $\Upsilon_{3}$ as a function of $(x, y)$. To apply the implicit function theorem we must check three properties:

(i) $\Upsilon_{3}$ is infinitely differentiable on $\mathcal{N}$.

(ii) For all $(\alpha, \beta) \in \mathcal{L},\left(x^{*}, y^{*}\right)$ is the only pair in $[2, \infty)^{2}$ satisfying $(\alpha, \beta, x, y) \in \mathcal{N}$ and $\Upsilon_{3}(\alpha, \beta, x, y)=0$.

(iii) For all $(\alpha, \beta) \in \mathcal{L}, J_{3} \neq 0$ in $\left(\alpha, \beta, x^{*}, y^{*}\right)$. 
It follows from Lemma 2.2.1(ii), Proposition 5.4.1 and (5.5.8) that property (i) and (ii) hold. To get property (iii), abbreviate $x \psi_{A B}(x)=\psi(x), y \kappa(y, 1)=\kappa(y)$. From Lemma 2.2.1(ii) and Proposition 5.4.1, we know that $\psi$ and $\kappa$ are infinitely differentiable. By (5.5.10),

$$
J_{3}=\frac{\partial^{2} V}{\partial x^{2}} \frac{\partial^{2} V}{\partial y^{2}}-\left(\frac{\partial^{2} V}{\partial x \partial y}\right)^{2} .
$$

Taking into account that $(\partial V / \partial x)\left(x^{*}, y^{*}\right)=(\partial V / \partial y)\left(x^{*}, y^{*}\right)=0$, we deduce from (5.5.8) that $\psi^{\prime}\left(x^{*}\right)=\kappa^{\prime}\left(y^{*}\right)$ and $J_{3}=c^{*} \psi^{\prime \prime}\left(x^{*}\right) \kappa^{\prime \prime}\left(y^{*}\right)$, where $c^{*}>0$ is a constant depending on $\left(x^{*}, y^{*}\right)$. We already know from Lemma 2.2.1(iii) that $\kappa^{\prime \prime}\left(y^{*}\right)<0$.

Lemma 5.5.2. $\psi^{\prime \prime}\left(x^{*}\right)<0$.

Proof. For $x>2$ satisfying $(\alpha, \beta, x) \in \mathcal{L}_{\psi}$, we will show that $\left(x \psi_{A B}(x)\right)^{\prime \prime}<0$. For this it suffices to show that there exists a $C>0$ such that, for $\delta$ small enough,

$$
T(\delta)=\frac{1}{2}\left[(x+\delta) \psi_{A B}(x+\delta)+(x-\delta) \psi_{A B}(x-\delta)-2 x \psi_{A B}(x)\right] \leq-C \delta^{2} .
$$

Set $x_{-\delta}=x-\delta$ and $x_{\delta}=x+\delta$, and let $\left(e_{-\delta}, b_{-\delta}\right)$ and $\left(e_{\delta}, b_{\delta}\right)$ be the unique maximisers of (2.3.6) at $x_{-\delta}$ and $x_{\delta}$. Pick $(c, b)=\left(\frac{1}{2}\left(e_{-\delta}+e_{\delta}\right), \frac{1}{2}\left(b_{-\delta}+b_{\delta}\right)\right)$ in (2.3.6). Since $x=\frac{1}{2}\left(x_{-\delta}+x_{\delta}\right)$, we obtain $T(\delta) \leq V_{1}(\delta)+V_{2}(\delta)$ with

$$
\begin{aligned}
V_{1}(\delta)= & \frac{1}{2}\left[e_{-\delta} \phi^{\mathcal{I}}\left(\frac{e_{-\delta}}{b_{-\delta}}\right)+e_{\delta} \phi^{\mathcal{I}}\left(\frac{e_{\delta}}{b_{\delta}}\right)-\left(e_{-\delta}+e_{\delta}\right) \phi^{\mathcal{I}}\left(\frac{e_{-\delta}+e_{\delta}}{b_{-\delta}+b_{\delta}}\right)\right], \\
V_{2}(\delta)= & \left(x_{-\delta}-e_{-\delta}\right) \kappa\left(x_{-\delta}-e_{-\delta}, 1-b_{-\delta}\right)+\left(x_{\delta}-e_{\delta}\right) \kappa\left(x_{\delta}-e_{\delta}, 1-b_{\delta}\right) \\
& -\left(x_{-\delta}+x_{\delta}-e_{-\delta}-e_{\delta}\right) \kappa\left(\frac{1}{2}\left(x_{-\delta}+x_{\delta}-e_{-\delta}-e_{\delta}\right), 1-\frac{1}{2}\left(b_{-\delta}+b_{\delta}\right)\right) .
\end{aligned}
$$

Lemma 5.5.3. The determinant of the Jacobian matrix of $(a, b) \mapsto a \kappa(a, b)$ is strictly positive everywhere on DOM.

Proof. The non-negativity of the Jacobian determinant is a consequence of the concavity of $(a, b) \mapsto a \kappa(a, b)$ (recall Lemma 2.2.1(ii)). The strict positivity can be checked with MAPLE via the explicit expression $\kappa(a, b)$ given in den Hollander and Whittington [7].

Since $(a, b) \mapsto a \kappa(a, b)$ is concave and twice differentiable, Lemma 5.5.3 allows us to assert that on DOM the Jacobian matrix of $(a, b) \mapsto a \kappa(a, b)$ has two strictly negative eigenvalues. The second derivatives of $\kappa$ are continuous. Moreover, the uniqueness of $\left(e_{-\delta}, b_{-\delta}\right)$ and $\left(e_{\delta}, b_{\delta}\right)$ imply their continuity in $\delta$, and so there exists a $\widetilde{C}>0$ such that, for $\delta$ small enough,

$$
V_{2}(\delta) \leq-\widetilde{C}\left[\left(\left(x_{-\delta}-x_{\delta}\right)-\left(e_{-\delta}-e_{\delta}\right)\right)^{2}+\left(b_{-\delta}-b_{\delta}\right)^{2}\right] .
$$


In what follows, we set $Y\left(\frac{e}{b}\right)=\left(\partial^{2} / \partial^{2} \mu\right)\left[\mu \phi^{\mathcal{I}}(\mu)\right]\left(\frac{e}{b}\right)$. To bound $V_{1}(\delta)$ from above, we compute the Jacobian matrix of $(e, b) \mapsto e \phi^{\mathcal{I}}(e / b)$ :

$$
\frac{1}{b} Y\left(\frac{e}{b}\right)\left(\begin{array}{cc}
1 & -\frac{e}{b} \\
-\frac{e}{b} & \frac{e^{2}}{b^{2}}
\end{array}\right) .
$$

Thus, if for $t \in[0,1]$ and $u \in[0, t]$ we set $e_{u, t}=\frac{e_{-\delta}+e_{\delta}}{2}+t\left(u-\frac{1}{2}\right)\left(e_{-\delta}-e_{\delta}\right)$ and $b_{u, t}=\frac{b_{-\delta}+b_{\delta}}{2}+t\left(u-\frac{1}{2}\right)\left(b_{-\delta}-b_{\delta}\right)$, then a Taylor expansion gives us

$$
V_{1}(\delta)=\frac{1}{4} \int_{0}^{1} d t t \int_{0}^{t} d u \frac{1}{b_{u, t}} Y\left(\frac{e_{u, t}}{b_{u, t}}\right)\left[\left(e_{-\delta}-e_{\delta}\right)-\frac{e_{u, t}}{b_{u, t}}\left(b_{-\delta}-b_{\delta}\right)\right]^{2} .
$$

As explained in the proof of Proposition 5.4.1, the fact that $(\alpha, \beta, x) \in \mathcal{L}_{\psi}$ implies $\left(e_{0}, b_{0}\right) \in \mathcal{L}_{\alpha, \beta, x}$ and therefore $\left(\alpha, \beta, \frac{e_{0}}{b_{0}}\right) \in \mathcal{L}_{\phi}$. Moreover, $\mathcal{L}_{\phi}$ is an open subset of CONE $\times[1, \infty)$ and $\left(e_{\delta}, b_{\delta}\right)$ is continuous in $\delta$, so that for $\delta$ small enough, $t \in[0,1]$ and $u \in[0, t]$, we have $\left(\alpha, \beta, \frac{e_{u, t}}{b_{u, t}}\right) \in \mathcal{L}_{\phi}$. This implies, by Lemma 5.3 .1 and by the continuity of the second derivative of $\phi^{\mathcal{I}}$ on $\mathcal{L}_{\phi}$, that there exists a $\widehat{C}>0$ such that, for $\delta$ small enough, $\frac{1}{b_{u, t}} Y\left(\frac{e_{u, t}}{b_{u, t}}\right) \leq-\widehat{C}$. At this stage, we need to consider the following three cases:

Case 1. $\left|b_{-\delta}-b_{\delta}\right| \geq \frac{b_{0}}{e_{0}} \frac{\delta}{4}$. Then, (5.5.14) gives $V_{2}(\delta) \leq-\frac{\widetilde{C} b_{0}^{2}}{4^{2} e_{0}^{2}} \delta^{2}$.

Case 2. $\left|e_{-\delta}-e_{\delta}\right| \leq \delta$. Then, since $x_{\delta}-x_{-\delta}=2 \delta$, (5.5.14) gives $V_{2}(\delta) \leq-\widetilde{C} \delta^{2}$.

Case 3. $\left|e_{-\delta}-e_{\delta}\right|>\delta$ and $\left|b_{-\delta}-b_{\delta}\right|<\frac{b_{0}}{e_{0}} \frac{\delta}{4}$. By continuity of $e_{\delta}$ and $b_{\delta}, \frac{e_{u, t}}{b_{u, t}} \leq \frac{2 e_{0}}{b_{0}}$ for $\delta$ small enough and therefore

$$
\left|\left(e_{-\delta}-e_{\delta}\right)-\frac{e_{u, t}}{b_{u, t}}\left(b_{-\delta}-b_{\delta}\right)\right| \geq\left|e_{-\delta}-e_{\delta}\right|-\frac{2 e_{0}}{b_{0}}\left|b_{-\delta}-b_{\delta}\right| \geq \delta-\frac{2 e_{0}}{b_{0}} \frac{b_{0}}{e_{0}} \frac{\delta}{4}=\frac{\delta}{2} .
$$

Thus, (5.5.16) and (5.5.17) give $V_{1}(\delta) \leq-\frac{\widehat{C}}{48} \delta^{2}$.

We conclude by setting $C=\min \left\{\frac{\widetilde{C} b_{0}^{2}}{4^{2} e_{0}^{2}}, \widetilde{C}, \frac{\widehat{C}}{48}\right\}$, so that Cases 1,2 and 3 give $T(\delta) \leq$ $-C \delta^{2}$ for $\delta$ small enough, which proves (5.5.12).

Lemma 5.5.2 implies that $J_{3}>0$. Hence, the implicit function theorem can indeed be applied to (5.5.8), and it follows that $f$ is infinitely differentiable on $\mathcal{L}$.

Acknowledgement. NP is supported by a postdoctoral fellowship from the Netherlands Organization for Scientific Research (grant 613.000.438). FdH and NP are grateful to the Pacific Institute for the Mathematical Sciences and the Mathematics Department of the University of British Columbia, Vancouver, Canada, for hospitality: FdH from January to August 2006, NP from mid-March to mid-April 2006 when the work in this paper started.

Open Access This article is distributed under the terms of the Creative Commons Attribution Noncommercial License which permits any noncommercial use, distribution, and reproduction in any medium, provided the original author(s) and source are credited.

\section{References}

1. Bredon, G.E.: Topology and Geometry. New York: Springer, 1993

2. Dembo, A., Zeitouni, O.: Large Deviations Techniques and Applications, 2nd ed., Applications of Mathematics 38, New York: Springer, 1998 
3. Caravenna, F.: Random Walk Models and Probabilistic Techniques for Inhomogeneous Polymer Chains, PhD Thesis, 21 October 2005, University of Milano-Bicocca, Italy, and University of Paris 7, France

4. Giacomin, G.: Random Polymer Models. London: Imperial College Press, 2007

5. Giacomin, G., Toninelli, F.L.: Smoothing effect of quenched disorder on polymer depinning transitions. Commun. Math. Phys. 266, 1-16 (2006)

6. Giacomin, G., Toninelli, F.L.: The localized phase of disordered copolymers with adsorption. ALEA 1, 149-180 (2006)

7. den Hollander, F., Whittington, S.G.: Localization transition for a copolymer in an emulsion. Theor. Prob. Appl. 51, 193-240 (2006)

8. Kingman, J.F.C.: Subadditive ergodic theory. Ann. Probab. 6, 883-909 (1973)

9. Pétrélis, N.: Localisation d'un Polymère en Interaction avec une Interface. PhD thesis, 2 February 2006, University of Rouen, France

Communicated by F. Toninelli 